\title{
QUASIPOTENTIAL AND EXIT TIME FOR 2D STOCHASTIC NAVIER-STOKES EQUATIONS DRIVEN BY SPACE TIME WHITE NOISE
}

\author{
Z. BRZEŹNIAK AND S. CERRAI AND M. FREIDLIN
}

\begin{abstract}
We are dealing with the Navier-Stokes equation in a bounded regular domain $\mathcal{O}$ of $\mathbb{R}^{2}$, perturbed by an additive Gaussian noise $\partial w^{Q_{\delta}} / \partial t$, which is white in time and colored in space. We assume that the correlation radius of the noise gets smaller and smaller as $\delta \searrow 0$, so that the noise converges to the white noise in space and time. For every $\delta>0$ we introduce the large deviation action functional $S_{T}^{\delta}$ and the corresponding quasi-potential $U_{\delta}$ and, by using arguments from relaxation and $\Gamma$-convergence we show that $U_{\delta}$ converges to $U=U_{0}$, in spite of the fact that the Navier-Stokes equation has no meaning in the space of square integrable functions, when perturbed by space-time white noise. Moreover, in the case of periodic boundary conditions the limiting functional $U$ is explicitly computed.

Finally, we apply these results to estimate of the asymptotics of the expected exit time of the solution of the stochastic Navier-Stokes equation from a basin of attraction of an asymptotically stable point for the unperturbed system.
\end{abstract}

\section{Contents}

1. Introduction

Acknowledgments

2. Notation and preliminaries 5

3. The skeleton equation 10

4. Some basic facts on relaxation and $\Gamma$-convergence 18

5. The large deviation action functional 19

6. The quasi-potential $\quad 24$

7. Stochastic Navier Stokes equations with periodic boundary conditions 29

8. Convergence of $U_{\delta}$ to $U$

9. Application to the exit problem 33

Appendix A. Proofs of some auxiliary results $\quad 36$

Appendix B. Proofs of Lemmas in Section 9 42

References

\section{INTRODUCTION}

Date: August 21, 2018. 
Let $\mathcal{O}$ be a regular bounded open domain of $\mathbb{R}^{2}$. We consider here the 2-dimensional NavierStokes equation in $\mathcal{O}$, perturbed by a small Gaussian noise

$$
\frac{\partial u(t, x)}{\partial t}=\Delta u(t, x)-(u(t, x) \cdot \nabla u(t, x)) u(t, x)-\nabla p(t, x)+\sqrt{\varepsilon} \eta(t, x),
$$

with the incompressibility condition

$$
\operatorname{div} u(t, x)=0
$$

and initial and boundary conditions

$$
u(t, x)=0, \quad x \in \partial \mathcal{O}, \quad u(0, x)=u_{0}(x) .
$$

Here $0<\varepsilon<<1$ and $\eta(t, x)$ is a Gaussian random field, white in time and colored in space. In what follows, for any $\alpha \in \mathbb{R}$ we shall denote by $\mathrm{V}_{\alpha}$ the closure in the space $\left[H^{\alpha}(\mathcal{O})\right]^{2}$ of the set of infinitely differentiable 2-dimensional vector fields, having zero divergence and compact support on $\mathcal{O}$, and we shall set $H=V_{0}$ and $V=V_{1}$. We will also set

$$
D(\mathrm{~A})=\left[H^{2}(\mathcal{O})\right]^{2} \cap \mathrm{V}, \quad \mathrm{A} x=-\Delta x, \quad x \in D(\mathrm{~A}) .
$$

The operator $\mathrm{A}$ is positive and self-adjoint, with compact resolvent. We will denote with $0<\lambda_{1} \leq \lambda_{2} \leq \cdots$ and $\left\{e_{k}\right\}_{k \in \mathbb{N}}$ the eigenvalues and the eigenfunctions of $\mathrm{A}$, respectively. Moreover, we will define the bilinear operator $B: \mathrm{V} \times \mathrm{V} \rightarrow \mathrm{V}_{-1}$ by setting

$$
\langle B(u, v), z\rangle=\int_{\mathcal{O}} z(x) \cdot[(u(x) \cdot \nabla) v(x)] d x .
$$

With these notations, if we apply to each term of the Navier-Stokes equation above the projection operator into the space of divergence free fields, we formally arrive to the abstract equation

$$
d u(t)+\mathrm{A} u(t)+B(u(t), u(t))=\sqrt{\varepsilon} d w^{Q}(t), \quad u(0)=u_{0},
$$

where the noise $w^{Q}(t)$ is assumed to be of the following form

$$
w^{Q}(t)=\sum_{k=1}^{\infty} Q e_{k} \beta_{k}(t), \quad t \geq 0,
$$

for some sequence of independent standard Brownian motions $\left\{\beta_{k}(t)\right\}_{k \in \mathbb{N}}$ and a linear operator $Q$ defined on $\mathrm{H}$ (for all details see Section 2).

As well known, white noise in space and time (that is $Q=I$ ) cannot be taken into consideration in order to study equation (1.1) in the space H. But if we assume that $Q$ is a compact operator satisfying suitable conditions, as for example $Q \sim \mathrm{A}^{-\alpha}$, for some $\alpha>0$, we have that for any $u_{0} \in \mathrm{H}$ and $T>0$ equation (1.1) is well defined in $C([0, T] ; \mathrm{H})$ and the validity of a large deviation principle and the problem of the exit of the solution of equation (1.1) from a domain can be studied.

As in the previous work [11, where a class of reaction-diffusion equations in any space dimension perturbed by multiplicative noise has been considered, in the present paper we want to see how we can describe the small noise asymptotics of equation (1.1), as if the noisy perturbation were given by a white noise in space and time. This means that, in spite of the fact that equation (1.1) is not meaningful in $\mathrm{H}$ when the noise is white in space, the relevant quantities for the large deviations and the exit problems associated with it can be approximated by the analogous quantities that one would get in the case of white noise in 
space. In particular, when periodic boundary conditions are imposed, such quantities can be explicitly computed and such approximation becomes particularly useful.

In what follows we shall consider a family of positive linear operators $\left\{Q_{\delta}\right\}_{\delta \in(0,1]}$ defined on $\mathrm{H}$, such that for any fixed $\delta \in(0,1]$ equation (1.1), with noise

$$
w^{Q_{\delta}}(t)=\sum_{k=1}^{\infty} Q_{\delta} e_{k} \beta_{k}(t), \quad t \geq 0
$$

is well defined in $C([0, T] ; \mathrm{H})$, and $Q_{\delta}$ is strongly convergent to the identity operator in $\mathrm{H}$, for $\delta \searrow 0$. For each fixed $\delta \in(0,1]$, the family $\left\{\mathcal{L}\left(u_{\varepsilon, \delta}^{x}\right)\right\}_{\varepsilon \in(0,1]}$ satisfies a large deviation principle in $C([0, T] ; \mathrm{H})$ with action functional

$$
S_{T}^{\delta}(u)=\frac{1}{2} \int_{0}^{T}\left|Q_{\delta}^{-1}\left(u^{\prime}(t)+\mathrm{A} u(t)+B(u(t), u(t))\right)\right|_{\mathrm{H}}^{2} d t,
$$

and the corresponding quasi-potential is defined by

$$
U_{\delta}(\phi)=\inf \left\{S_{T}^{\delta}(u): u \in C([0, T] ; \mathrm{H}), u(0)=0, u(T)=\phi, T>0\right\} .
$$

Our purpose here is to show that, despite we cannot prove any limit for the solution $u^{\delta}$ of equation (1.1), nevertheless, for all $\phi \in \mathrm{H}$ such that $U_{\delta}(\phi)<\infty$,

$$
\lim _{\delta \rightarrow 0} U_{\delta}(\phi)=U(\phi)
$$

where $U(\phi)$ is defined as $U_{\delta}(\phi)$, with the action functional $S_{T}^{\delta}$ replaced by

$$
S_{T}(u)=\frac{1}{2} \int_{0}^{T}\left|u^{\prime}(t)+\mathrm{A} u(t)+B(u(t), u(t))\right|_{\mathrm{H}}^{2} d t .
$$

To this purpose, the key idea consists in characterizing the quasi-potentials $U_{\delta}$ and $U$ as

$$
U_{\delta}(\phi)=\min \left\{S_{-\infty}^{\delta}(u): u \in \mathcal{X} \text { and } u(0)=\phi\right\}
$$

and

where

$$
U(\phi)=\min \left\{S_{-\infty}(u): u \in \mathcal{X} \text { and } u(0)=\phi\right\}
$$

$$
\mathcal{X}=\left\{u \in C((-\infty, 0] ; \mathrm{H}): \lim _{t \rightarrow-\infty}|u(t)|_{\mathrm{H}}=0\right\}
$$

and the functionals $S_{-\infty}^{\delta}$ and $S_{-\infty}$ are defined on $\mathcal{X}$ in a natural way, see formulae (5.6) and (5.5) later on.

In this way, in the definition of $U_{\delta}$ and $U$, the infimum with respect to time $T>0$ has disappeared and we have only to take the infimum of suitable functionals in the space $\mathcal{X}_{\phi}:=$ $\{u \in \mathcal{X}: u(0)=\phi\}$. In particular, the convergence of $U_{\delta}(\phi)$ to $U(\phi)$ becomes the convergence of the infima of $S_{-\infty}^{\delta}$ in $\mathcal{X}_{\phi}$ to the infimum of $S_{-\infty}$ in $\mathcal{X}_{\phi}$, so that (1.3) follows once we prove that $S_{-\infty}^{\delta}$ is Gamma-convergent to $S_{-\infty}$ in $\mathcal{X}_{\phi}$, as $\delta \searrow 0$. Moreover, as a consequence of (1.5), in the case of the stochastic Navier-Stokes equations with periodic boundary conditions we can prove, see section 7 , that

$$
U(\phi)=|\phi|_{\mathrm{V}}^{2}
$$

This means that $U(\phi)$ can be explicitly computed and the use of (1.3) in applications becomes particularly relevant. Let us point out that a similar explicit formula for the quasipotential 
has been derived for linear SPDEs by Da Prato, Pritchard and Zabczyk in [17] and in the recent work by the second and third authors for stochastic reaction diffusion equations in [11. A finite dimensional counterpart of our formula (1.6) was first derived in Theorem IV.3.1 in the monograph [20].

The proofs of characterizations (1.4) and (1.5) and of the Gamma-convergence of $S_{-\infty}^{\delta}$ to $S_{-\infty}$ are based on a thorough analysis of the Navier-Stokes equation with an external deterministic force in the domain of suitable fractional powers of the operator A.

One of the main motivation for proving (1.3) comes from the study of the expected exit time $\tau_{\phi}^{\varepsilon, \delta}$ of the solution $u_{\phi}^{\varepsilon, \delta}$ from a domain $D$ in $L^{2}(\mathcal{O})$, which is attracted to the zero function. Actually, in the second part of the paper we prove that, under suitable regularity properties of $D$, for any fixed $\delta>0$

$$
\lim _{\varepsilon \rightarrow 0} \varepsilon \log \mathbb{E}\left(\tau_{\phi}^{\varepsilon, \delta}\right)=\inf _{y \in \partial D} U_{\delta}(y)
$$

This means that, as in finite dimension, the expectation of $\tau_{\phi}^{\varepsilon, \delta}$ can be described in terms of the quantity $U_{\delta}(\phi)$. Moreover, once we have (1.3), by a general argument introduced in [11] and based again on Gamma-convergence, we can prove that if $D$ is a domain in $\mathrm{H}$ such that any point $\phi \in \mathrm{V} \cap \partial D$ can be approximated in $\mathrm{V}$ by a sequence $\left\{\phi_{n}\right\}_{n \in \mathbb{N}} \subset D\left(\mathrm{~A}^{\frac{1}{2}+\alpha}\right) \cap \partial D$ (think for example of $D$ as a ball in $\mathrm{H}$ ), then

$$
\lim _{\delta \rightarrow 0} \inf _{\phi \in \partial D} U_{\delta}(\phi)=\inf _{\phi \in \partial D} U(\phi) .
$$

According to (1.7), this implies that for $0<\varepsilon<<\delta<<1$

$$
\mathbb{E} \tau_{\phi}^{\varepsilon, \delta} \sim \exp \left(\frac{1}{\varepsilon} \inf _{\phi \in \partial D} U(\phi)\right) .
$$

In particular, if $D$ is the ball of $\mathrm{H}$ of radius $c$ and the boundary conditions are periodic, in view of (1.6) for any $\phi \in D$ we get,

$$
e^{-\frac{c^{2} \lambda_{1}^{2}}{\varepsilon}} \mathbb{E} \tau_{\phi}^{\varepsilon, \delta} \sim 1, \quad 0<\varepsilon<<\delta<<1 .
$$

At the end of this long introduction, we would like to point out that although 2-D stochastic Navier-Stokes equations with periodic boundary conditions have been investigated by Flandoli and Gozzi in [23] and Da Prato and Debussche in [16] from the point of view of Kolmogorov equations and the existence of a Markov process, we do not know whether our results (even in the periodic case) could be derived from these papers. One should bear in mind that the solution from [16] exists for almost every initial data $u_{0}$ from a certain Besov space of negative order with respect to a specific Gaussian measure while we construct a quasipotential for every $u_{0}$ from the space $\mathrm{H}$ whose measure is equal to 0 . Of course our results are also valid for 2-D stochastic Navier-Stokes equations with Dirichlet boundary conditions.

We have been recently become aware of a work by F. Bouchet et all [3] where somehow related issues are considered from a physical point of view. We hope to be able to understand the relationship between our work and this work in a future publication. 
Acknowledgments. Research of the first named author was also supported by the EPSRC grant EP/E01822X/1, research of the second named author was also supported by NSF grant DMS0907295 and research of the third named author was also supported by the NSF grants DMS 0803287 and 0854982.

The first named author would like to thank the University of Maryland for hospitality during his visit in Summer 2008. The second named author would like to thank Michael Salins for some useful discussions about Lemmas 9.8 and 9.9 .

Finally, the three authors would like to thank the two anonymous referees for reading carefully the original manuscript and giving very good suggestions that helped us to improve considerably the final version of the paper.

\section{Notation AND PRELIMINARIES}

Let $\mathcal{O} \subset \mathbb{R}^{2}$ be an open and bounded set. We denote by $\Gamma=\partial \mathcal{O}$ the boundary of $\mathcal{O}$. We will always assume that the closure $\overline{\mathcal{O}}$ of the set $\mathcal{O}$ is a manifold with boundary of $C^{\infty}$ class, whose boundary $\partial \mathcal{O}$ is denoted by $\Gamma$, is a 1-dimensional infinitely differentiable manifold being locally on one side of $\mathcal{O}$, see condition (7.10) from [30, chapter I]. Let us also denote by $\nu$ the unit outer normal vector field to $\Gamma$.

It is known that $\mathcal{O}$ is a Poincaré domain, i.e. there exists a constant $\lambda_{1}>0$ such that the following Poincaré inequality is satisfied

$$
\lambda_{1} \int_{\mathcal{O}} \varphi^{2}(x) d x \leq \int_{\mathcal{O}}|\nabla \varphi(x)|^{2} d x, \quad \varphi \in H_{0}^{1}(\mathcal{O}) .
$$

In order to formulate our problem in an abstract framework, let us recall the definition of the following functional spaces. First of all, let $\mathcal{D}(\mathcal{O})(\operatorname{resp} . \mathcal{D}(\overline{\mathcal{O}}))$ be the set of all $C^{\infty}$ class vector fields $u: \mathbb{R}^{2} \rightarrow \mathbb{R}^{2}$ with compact support contained in the set $\mathcal{O}$ (resp. $\overline{\mathcal{O}}$ ). Then, let us define

$$
\begin{aligned}
E(\mathcal{O}) & =\left\{u \in L^{2}\left(\mathcal{O}, \mathbb{R}^{2}\right): \operatorname{div} u \in L^{2}(\mathcal{O})\right\} \\
\mathcal{V} & =\{u \in \mathcal{D}(\mathcal{O}): \operatorname{div} u=0\} \\
\mathrm{H} & =\text { the closure of } \mathcal{V} \text { in } L^{2}(\mathcal{O}) \\
\mathrm{H}_{0}^{1}\left(\mathcal{O}, \mathbb{R}^{2}\right) & =\text { the closure of } \mathcal{D}\left(\mathcal{O}, \mathbb{R}^{2}\right) \text { in } \mathrm{H}^{1}\left(\mathcal{O}, \mathbb{R}^{2}\right), \\
V & =\text { the closure of } \mathcal{V} \text { in } \mathrm{H}_{0}^{1}\left(\mathcal{O}, \mathbb{R}^{2}\right) .
\end{aligned}
$$

The inner products in all the $L^{2}$ spaces will be denoted by $(\cdot, \cdot)$. The space $E(\mathcal{O})$ is a Hilbert space with a scalar product

$$
\langle u, v\rangle_{E(\mathcal{O})}:=(u, v)_{L^{2}\left(\mathcal{O}, \mathbb{R}^{2}\right)}+(\operatorname{div} u, \operatorname{div} v)_{L^{2}\left(\mathcal{O}, \mathbb{R}^{2}\right)} .
$$

We endow the set $H$ with the inner product $(\cdot, \cdot)_{H}$ and the norm $|\cdot|_{H}$ induced by $L^{2}\left(\mathcal{O}, \mathbb{R}^{2}\right)$. Thus, we have

$$
(u, v)_{H}=\sum_{j=1}^{2} \int_{\mathcal{O}} u_{j}(x) v_{j}(x) d x,
$$

The space $H$ can also be characterised in the following way. Let $H^{-\frac{1}{2}}(\Gamma)$ be the dual space of $H^{1 / 2}(\Gamma)$, the image in $L^{2}(\Gamma)$ of the trace operator $\gamma_{0}: H^{1}(\mathcal{O}) \rightarrow L^{2}(\Gamma)$ and let $\gamma_{\nu}$ be the 
bounded linear map from $E(\mathcal{O})$ to $H^{-\frac{1}{2}}(\Gamma)$ such that, see [42, Theorem I.1.2],

$$
\gamma_{\nu}(u)=\text { the restriction of } u \cdot \nu \text { to } \Gamma, \quad \text { if } u \in \mathcal{D}(\overline{\mathcal{O}}) \text {. }
$$

Then, see [42, Theorem I.1.4],

$$
\begin{aligned}
& \mathrm{H}=\left\{u \in E(\mathcal{O}): \operatorname{div} u=0 \text { and } \gamma_{\nu}(u)=0\right\}, \\
& \mathrm{H}^{\perp}=\left\{u \in E(\mathcal{O}): u=\nabla p, p \in H^{1}(\mathcal{O})\right\} .
\end{aligned}
$$

Let us denote by $\mathrm{P}: L^{2}\left(\mathcal{O}, \mathbb{R}^{2}\right) \rightarrow \mathrm{H}$ the orthogonal projection called usually the LerayHelmholtz projection. It is known, see for instance [42, Remark I.1.6] that

$$
P u=u-\nabla(p+q), \quad u \in L^{2}\left(\mathcal{O}, \mathbb{R}^{2}\right),
$$

where, for $u \in L^{2}(\mathcal{O}), p$ is the unique solution of the following homogenous boundary Dirichlet problem for the Laplace equation

$$
\Delta p=\operatorname{div} u \in H^{-1}(\mathcal{O}),\left.\quad p\right|_{\Gamma}=0 .
$$

and $q \in H^{1}(\mathcal{O})$ is the unique solution of the following in-homogenous Neumann boundary problem for the Laplace equation

$$
\Delta q=0,\left.\quad \frac{\partial q}{\partial \nu}\right|_{\Gamma}=\gamma_{\nu}(u-\nabla p)
$$

Note that the function $p$ above satisfies $\nabla p \in L^{2}\left(\mathcal{O}, \mathbb{R}^{2}\right)$ and $\operatorname{div}(u-\nabla p)=0$. In particular, $u-\nabla p \in E(\mathcal{O})$ so that $q$ is well defined.

It is proved in [42, Remark I.1.6] that $P$ maps continuously the Sobolev space $\mathrm{H}^{1}\left(\mathcal{O}, \mathbb{R}^{2}\right)$ into itself. Below, we will discuss continuity of $P$ with respect to other topologies.

Since the set $\mathcal{O}$ is a Poincare domain, the norms on the space $\mathrm{V}$ induced by norms from the Sobolev spaces $\mathrm{H}^{1}\left(\mathcal{O}, \mathbb{R}^{2}\right)$ and $\mathrm{H}_{0}^{1}\left(\mathcal{O}, \mathbb{R}^{2}\right)$ are equivalent. The latter norm and the associated inner product will be denoted by $|\cdot|_{\mathrm{V}}$ and $(\cdot, \cdot)_{\mathrm{V}}$, respectively. They satisfy the following equality

$$
(u, v)_{\mathrm{V}}=\sum_{i, j=1}^{2} \int_{\mathcal{O}} \frac{\partial u_{j}}{\partial x_{i}} \frac{\partial v_{j}}{\partial x_{i}} d x, \quad u, v \in \mathrm{H}_{0}^{1}\left(\mathcal{O}, \mathbb{R}^{2}\right) .
$$

Since the space $\mathrm{V}$ is densely and continuously embedded into $\mathrm{H}$, by identifying $\mathrm{H}$ with its dual $\mathrm{H}^{\prime}$, we have the following embeddings

$$
\mathrm{V} \subset \mathrm{H} \cong \mathrm{H}^{\prime} \subset \mathrm{V}^{\prime}
$$

Let us observe here that, in particular, the spaces $\mathrm{V}, \mathrm{H}$ and $\mathrm{V}^{\prime}$ form a Gelfand triple.

We will denote by $|\cdot|_{\mathrm{V}^{\prime}}$ and $\langle\cdot, \cdot\rangle$ the norm in $\mathrm{V}^{\prime}$ and the duality pairing between $\mathrm{V}$ and $\mathrm{V}^{\prime}$, respectively.

The presentation of the Stokes operator is standard and we follow here the one given in [9]. We first define the bilinear form $a: \mathrm{V} \times \mathrm{V} \rightarrow \mathbb{R}$ by setting

$$
a(u, v):=(\nabla u, \nabla v)_{\mathrm{H}}, \quad u, v \in \mathrm{V} .
$$

As obviously the bilinear form $a$ coincides with the scalar product in $\mathrm{V}$, it is $\mathrm{V}$-continuous, i.e. there exists some $C>0$ such that

$$
|a(u, u)| \leq C|u|_{\mathrm{V}}^{2}, \quad u \in \mathrm{V}
$$


Hence, by the Riesz Lemma, there exists a unique linear operator $\mathcal{A}: \mathrm{V} \rightarrow \mathrm{V}^{\prime}$, such that $a(u, v)=\langle\mathcal{A} u, v\rangle$, for $u, v \in \mathrm{V}$. Moreover, since $\mathcal{O}$ is a Poincaré domain, the form $a$ is V-coercive, i.e. it satisfies $a(u, u) \geq \alpha|u|_{\mathrm{V}}^{2}$ for some $\alpha>0$ and all $u \in \mathrm{V}$. Therefore, in view of the Lax-Milgram theorem, see for instance Temam [42, Theorem II.2.1], the operator $\mathcal{A}: \mathrm{V} \rightarrow \mathrm{V}^{\prime}$ is an isomorphism.

Next we define an unbounded linear operator $\mathrm{A}$ in $\mathrm{H}$ as follows

$$
\left\{\begin{array}{l}
D(\mathrm{~A})=\{u \in \mathrm{V}: \mathcal{A} u \in \mathrm{H}\} \\
\mathrm{A} u \quad=\mathcal{A} u, u \in D(\mathrm{~A}) .
\end{array}\right.
$$

It is now well established that under suitable assumptions related to the regularity of the domain $\mathcal{O}$, the space $D(\mathrm{~A})$ can be characterized in terms of the Sobolev spaces. For example, (see [27, where only the 2-dimensional case is studied but the result is also valid in the 3-dimensional case), if $\mathcal{O} \subset \mathbb{R}^{2}$ is a uniform $C^{2}$-class Poincaré domain, then we have

$$
\left\{\begin{array}{l}
D(\mathrm{~A})=\mathrm{V} \cap \mathrm{H}^{2}\left(\mathcal{O}, \mathbb{R}^{2}\right)=\mathrm{H} \cap \mathrm{H}_{0}^{1}\left(\mathcal{O}, \mathbb{R}^{2}\right) \cap \mathrm{H}^{2}\left(\mathcal{O}, \mathbb{R}^{2}\right), \\
\mathrm{A} u=-\mathrm{P} \Delta u, \quad u \in D(\mathrm{~A}) .
\end{array}\right.
$$

It is also a classical result, see e.g. Cattabriga [13] or Temam [41, p. 56], that A is a positive self adjoint operator in $\mathrm{H}$ and

$$
(\mathrm{A} u, u) \geq \lambda_{1}|u|_{\mathrm{H}}^{2}, \quad u \in D(\mathrm{~A}) .
$$

where the constant $\lambda_{1}>0$ is from the Poincaré inequality (2.1). Moreover, it is well known, see for instance [41, p. 57] that $\mathrm{V}=D\left(\mathrm{~A}^{1 / 2}\right)$. Moreover, from [44, Theorem 1.15.3, p. 103] it follows that

$$
D\left(\mathrm{~A}^{\alpha / 2}\right)=[\mathrm{H}, D(\mathrm{~A})]_{\frac{\alpha}{2}}
$$

where $[\cdot, \cdot]_{\frac{\alpha}{2}}$ is the complex interpolation functor of order $\frac{\alpha}{2}$, see e.g. [30], 44, and [38, Theorem 4.2]. Furthermore, as shown in [44, Section 4.4.3], for $\alpha \in\left(0, \frac{1}{2}\right)$

$$
D\left(\mathrm{~A}^{\alpha / 2}\right)=\mathrm{H} \cap \mathrm{H}^{\alpha}\left(\mathcal{O}, \mathbb{R}^{2}\right) .
$$

The above equality leads to the following result.

Proposition 2.1. Assume that $\alpha \in\left(0, \frac{1}{2}\right)$. Then the Leray-Helmholtz projection $P$ is a well defined and continuous map from $\mathrm{H}^{\alpha}\left(\mathcal{O}, \mathbb{R}^{2}\right)$ into $D\left(\mathrm{~A}^{\alpha / 2}\right)$.

Proof. Let us fix $\alpha \in\left(0, \frac{1}{2}\right)$. Since, by its definition, the range of $P$ is contained in $\mathrm{H}$, it is sufficient to prove that for every $u \in \mathrm{H}^{\alpha}\left(\mathcal{O}, \mathbb{R}^{2}\right), P u \in \mathrm{H}^{\alpha}\left(\mathcal{O}, \mathbb{R}^{2}\right)$. For this aim, let us fix $u \in \mathrm{H}^{\alpha}\left(\mathcal{O}, \mathbb{R}^{2}\right)$. Then $\operatorname{div} u \in H^{\alpha-1}(\mathcal{O})$. Therefore, by the elliptic regularity we infer that the solution $p$ of the problem (2.5) belongs to the Sobolev space $H^{\alpha+1}(\mathcal{O}) \cap H_{0}^{1}(\mathcal{O})$ and therefore $\nabla p \in \mathrm{H}^{\alpha}\left(\mathcal{O}, \mathbb{R}^{2}\right)$.

Since by [42, Theorem I.1.2], the linear map $\gamma_{\nu}$ is bounded from $E(\mathcal{O})$ to $H^{-\frac{1}{2}}(\Gamma)$ and from $E(\mathcal{O}) \cap \mathrm{H}^{1}\left(\mathcal{O}, \mathbb{R}^{2}\right)$ to $H^{\frac{1}{2}}(\Gamma)$, by a standard interpolation argument we infer that $\gamma_{\nu}$ is a bounded linear map from $E(\mathcal{O}) \cap \mathrm{H}^{\alpha}\left(\mathcal{O}, \mathbb{R}^{2}\right)$ to $H^{-\frac{1}{2}+\alpha}(\Gamma)$. Thus we infer that $\gamma_{\nu}(u-\nabla p) \in$

\footnotetext{
${ }^{1}$ These assumptions are satisfied in our case
} 
$H^{-\frac{1}{2}+\alpha}(\Gamma)$ and by the Stokes formula (I.1.19) from [42], $\left\langle\gamma_{\nu}(u-\nabla p), 1\right\rangle=0$. Therefore, again by the elliptic regularity, see for instance [30], the solution $q$ of the problem (2.6) belongs to $H^{\alpha+1}(\mathcal{O})$ and therefore $\nabla q \in \mathrm{H}^{\alpha}\left(\mathcal{O}, \mathbb{R}^{2}\right)$. This proves that $P u \in \mathrm{H}^{\alpha}\left(\mathcal{O}, \mathbb{R}^{2}\right)$ as required.

The proof is complete.

Remark 2.2. We only claim that the above result is true for $\alpha<\frac{1}{2}$. In particular, we do not claim that is $P$ is a bounded linear map from $\mathrm{H}^{1}\left(\mathcal{O}, \mathbb{R}^{2}\right)$ to $\mathrm{V}$ and we are not aware of such a result. However, if this is true, Proposition 2.1 will hold for any $\alpha \in(0,1)$ with a simple proof by complex interpolation. However, it seems to us that the result for $\alpha>\frac{1}{2}$ is not true, since we cannot see how one could prove that $\left.P u\right|_{\partial \mathcal{O}}=0$. The reason why Proposition 2.1 holds for any $\alpha \in\left(0, \frac{1}{2}\right)$ is that according to identity (2.12) the only boundary conditions satisfied by functions belonging to $D\left(\mathrm{~A}^{\alpha / 2}\right)$ are those satisfied by functions belonging to the space $\mathrm{H}$. One can compare with the paper [39] by Temam (or chapter 6 of his book [40]).

Let us finally recall that by a result of Fujiwara-Morimoto 24] the projection $\mathrm{P}$ extends to a bounded linear projection in the space $L^{q}\left(\mathcal{O}, \mathbb{R}^{2}\right)$, for any $q \in(1, \infty)$.

Now, consider the trilinear form $b$ on $V \times V \times V$ given by

$$
b(u, v, w)=\sum_{i, j=1}^{2} \int_{\mathcal{O}} u_{i} \frac{\partial v_{j}}{\partial x_{i}} w_{j} d x, \quad u, v, w \in \mathrm{V} .
$$

Indeed, $b$ is a continuous trilinear form such that

$$
b(u, v, w)=-b(u, w, v), \quad u \in \mathrm{V}, v, w \in \mathrm{H}_{0}^{1}\left(\mathcal{O}, \mathbb{R}^{2}\right),
$$

and

$$
|b(u, v, w)| \leq C \begin{cases}|u|_{\mathrm{H}}^{1 / 2}|\nabla u|_{\mathrm{H}}^{1 / 2}|\nabla v|_{\mathrm{H}}^{1 / 2}|\mathrm{~A} v|_{\mathrm{H}}^{1 / 2}|w|_{\mathrm{H}} & u \in \mathrm{V}, v \in D(\mathrm{~A}), w \in \mathrm{H} \\ |u|_{\mathrm{H}}^{1 / 2}|\mathrm{~A} u|_{\mathrm{H}}^{1 / 2}|\nabla v|_{\mathrm{H}}|w|_{\mathrm{H}} & u \in D(\mathrm{~A}), v \in \mathrm{V}, w \in \mathrm{H} \\ |u|_{\mathrm{H}}|\nabla v|_{\mathrm{H}}|w|_{\mathrm{H}}^{1 / 2}|\mathrm{~A} w|_{\mathrm{H}}^{1 / 2} & u \in \mathrm{H}, v \in \mathrm{V}, w \in D(\mathrm{~A}) \\ |u|_{\mathrm{H}}^{1 / 2}|\nabla u|_{\mathrm{H}}^{1 / 2}|\nabla v|_{\mathrm{H}}|w|_{\mathrm{H}}^{1 / 2}|\nabla w|_{\mathrm{H}}^{1 / 2} & u, v, w \in \mathrm{V},\end{cases}
$$

for some constant $C>0$ (for a proof see for instance [42, Lemma 1.3, p.163] and [41]).

Define next the bilinear map $B: \mathrm{V} \times \mathrm{V} \rightarrow \mathrm{V}^{\prime}$ by setting

$$
\langle B(u, v), w\rangle=b(u, v, w), \quad u, v, w \in \mathrm{V},
$$

and the homogenous polynomial of second degree $B: \mathrm{V} \rightarrow \mathrm{V}^{\prime}$ by

$$
B(u)=B(u, u), u \in \mathrm{V} .
$$

Let us observe that if $v \in D(\mathrm{~A})$, then $B(u, v) \in H$ and the following inequality follows directly from the first inequality in (2.14)

$$
|\mathrm{B}(u, v)|_{\mathrm{H}}^{2} \leq C|u|_{\mathrm{H}}|\nabla u|_{\mathrm{H}}|\nabla v|_{\mathrm{H}}|\mathrm{A} v|_{\mathrm{H}}, u \in \mathrm{V}, v \in D(\mathrm{~A})
$$

Moreover, the following identity is a direct consequence of (2.13).

$$
\langle\mathrm{B}(u, v), v\rangle=0, \quad u, v \in \mathrm{V} .
$$

Let us also recall the following fact (see [9, Lemma 4.2]). 
Lemma 2.3. The trilinear map $b: \mathrm{V} \times \mathrm{V} \times \mathrm{V} \rightarrow \mathbb{R}$ has a unique extension to a bounded trilinear map from $L^{4}\left(\mathcal{O}, \mathbb{R}^{2}\right) \times\left(L^{4}\left(\mathcal{O}, \mathbb{R}^{2}\right) \cap \mathrm{H}\right) \times \mathrm{V}$ and from $L^{4}\left(\mathcal{O}, \mathbb{R}^{2}\right) \times \mathrm{V} \times L^{4}\left(\mathcal{O}, \mathbb{R}^{2}\right)$ into $\mathbb{R}$. Moreover, $B$ maps $L^{4}\left(\mathcal{O}, \mathbb{R}^{2}\right) \cap \mathrm{H}$ (and so $\mathrm{V}$ ) into $\mathrm{V}^{\prime}$ and

$$
|B(u)|_{\mathrm{V}^{\prime}} \leq C_{1}|u|_{L^{4}\left(\mathcal{O}, \mathbb{R}^{2}\right)}^{2} \leq 2^{1 / 2} C_{1}|u|_{\mathrm{H}}|\nabla u|_{\mathrm{H}} \leq C_{2}|u|_{\mathrm{V}}^{2}, \quad u \in \mathrm{V}
$$

Proof. It it enough to observe that due to the Hölder inequality, the following inequality holds

$$
|b(u, v, w)| \leq C|u|_{L^{4}\left(\mathcal{O}, \mathbb{R}^{2}\right)}|\nabla v|_{L^{2}(\mathcal{O})}|w|_{L^{4}\left(\mathcal{O}, \mathbb{R}^{2}\right)}, \quad u, v, w \in \mathrm{H}_{0}^{1}\left(\mathcal{O}, \mathbb{R}^{2}\right) .
$$

Thus, our result follows from (2.13).

Let us also recall the following well known result, see [42] for a proof.

Lemma 2.4. For any $T \in(0, \infty]$ and for any $u \in L^{2}(0, T ; D(\mathrm{~A}))$ with $u^{\prime} \in L^{2}(0, T ; \mathrm{H})$, we have

$$
\int_{0}^{T}|\mathrm{~B}(u(t), u(t))|_{\mathrm{H}}^{2} d t<\infty .
$$

Proof. Our assumption implies that'2 $u \in C([0, T] ; \mathrm{V})$ (for a proof see for instance [46, Proposition I.3.1]). Then, we can conclude thanks to (2.15)

The restriction of the map $\mathrm{B}$ to the space $D(\mathrm{~A}) \times D(\mathrm{~A})$ has also the following representation

$$
\mathrm{B}(u, v)=P(u \nabla v), u, v \in D(\mathrm{~A}),
$$

where $P$ is the Leray-Helmholtz projection operator and $u \nabla v=\sum_{j=1}^{2} u^{j} D_{j} v \in L^{2}\left(\mathcal{O}, \mathbb{R}^{2}\right)$. This representation together with Proposition 2.1 allows us to prove the following property of the map $B$.

Proposition 2.5. Assume that $\alpha \in\left(0, \frac{1}{2}\right)$. Then for any $s \in(1,2]$ there exists a constant $c>0$ such that

$$
|\mathrm{B}(u, v)|_{D\left(\mathrm{~A}^{\alpha / 2}\right)} \leq c|u|_{D\left(\mathrm{~A}^{\frac{s}{2}}\right)}|v|_{D\left(\mathrm{~A}^{\frac{1+\alpha}{2}}\right)}, \quad u, v \in D(\mathrm{~A}) .
$$

Proof. In view of equality (2.19), since the Leray-Helmholtz projection $P$ is a well defined and continuous map from $\mathrm{H}^{\alpha}\left(\mathcal{O}, \mathbb{R}^{2}\right)$ into $D\left(\mathrm{~A}^{\alpha / 2}\right)$ and since the norms in the spaces $D\left(\mathrm{~A}^{\frac{s}{2}}\right)$ are equivalent to norms in $\mathrm{H}^{s}\left(\mathcal{O}, \mathbb{R}^{2}\right)$, it is enough to show that

$$
|u \nabla v|_{\mathrm{H}^{\alpha}} \leq C|u|_{\mathrm{H}^{s}}|v|_{\mathrm{H}^{1+\alpha}}, \quad u, v \in \mathrm{H}^{2}\left(\mathcal{O}, \mathbb{R}^{2}\right) .
$$

The last inequality is a consequence of the Marcinkiewicz Interpolation Theorem, the complex interpolation and the following two inequalities for scalar functions which can be proved by using Gagliado-Nirenberg inequalities

$$
\begin{aligned}
& |u v|_{L^{2}} \leq C|u|_{H^{s}}|v|_{L^{2}}, \quad u \in H^{s}, v \in L^{2}, \\
& |u v|_{H^{1}} \leq C|u|_{H^{s}}|v|_{H^{1}}, \quad u \in H^{s}, v \in H^{1} .
\end{aligned}
$$

\footnotetext{
${ }^{2}$ Note that in the case $T=\infty$ one also has $\lim _{t \rightarrow \infty} u(t)=0$ in $\mathrm{V}$.
} 


\section{The SKeleton EQUATION}

We are here dealing with the following functional version of the Navier-Stokes equation

$$
\left\{\begin{array}{l}
u^{\prime}(t)+\nu \mathrm{A} u(t)+\mathrm{B}(u(t), u(t))=f(t), t \in(0, T) \\
u(0)=u_{0},
\end{array}\right.
$$

where $T \in(0, \infty]$ and $\nu>0$. Let us recall the following definition (see [42, Problem 2, section III.3])

Definition 3.1. Given $T>0, f \in L^{2}\left(0, T ; \mathrm{V}^{\prime}\right)$ and $u_{0} \in \mathrm{H}$, a solution to problem (3.1) is a function $u \in L^{2}(0, T ; \mathrm{V})$ such that $u^{\prime} \in L^{2}\left(0, T ; \mathrm{V}^{\prime}\right), u(0)=u_{0}$ 药 and (3.1) is fulfilled.

It is known (see e.g. [42, Theorems III.3.1/2]) that if $T \in(0, \infty]$, then for every $f \in$ $L^{2}\left(0, T ; \mathrm{V}^{\prime}\right)$ and $u_{0} \in \mathrm{H}$ there exists exactly one solution $u$ to problem (3.1), which satisfies

$$
|u|_{C([0, T], \mathrm{H})}^{2}+|u|_{L^{2}(0, T, \mathrm{~V})}^{2} \leq\left|u_{0}\right|_{\mathrm{H}}^{2}+|f|_{L^{2}\left(0, T, \mathrm{~V}^{\prime}\right)}^{2}
$$

Moreover, see [42, Theorem III.3.10], if $T<\infty$ and $f \in L^{2}(0, T ; H)$ then

$$
\begin{aligned}
& \sup _{t \in(0, T]}|\sqrt{t} u(t)|_{V}^{2}+\int_{0}^{T}|\sqrt{t} \mathrm{~A} u(t)|_{\mathrm{H}}^{2} d t \\
& \quad \leq\left(\left|u_{0}\right|_{\mathrm{H}}^{2}+|f|_{L^{2}\left(0, T ; \mathrm{V}^{\prime}\right)}^{2}+T|f|_{L^{2}(0, T ; \mathrm{H})}^{2}\right) e^{c\left(\left|u_{0}\right|_{\mathrm{H}}^{4}+|f|_{L^{2}\left(0, T ; \mathrm{V}^{\prime}\right)}^{4}\right)}
\end{aligned}
$$

for a constant $c$ independent of $T, f$ and $u_{0}$.

Finally, if $T \in(0, \infty], f \in L^{2}(0, T ; \mathrm{H})$ and $u_{0} \in \mathrm{V}$, then the unique solution $u$ satisfies

$$
u \in L^{2}(0, T ; D(\mathrm{~A})) \cap C([0, T] ; \mathrm{V}), \quad u^{\prime} \in L^{2}(0, T ; \mathrm{H}),
$$

and, for $t \in[0, T] \cap[0, \infty)$,

$$
\begin{aligned}
\frac{d}{d t}|u(t)|_{V}^{2}+\lambda_{1}|u(t)|_{V}^{2} & \leq \frac{d}{d t}|u(t)|_{V}^{2}+|\mathrm{A} u(t)|_{H}^{2} \\
& \leq 2|f(t)|_{\mathrm{H}}^{2}+108|u(t)|_{V}^{2}|u(t)|_{\mathrm{H}}^{2}|u(t)|_{V}^{2} .
\end{aligned}
$$

Hence, by the Gronwall Lemma and inequality (3.2), for any $t \in[0, T] \cap[0, \infty)$

$$
|u(t)|_{V}^{2} \leq\left(\left|u_{0}\right|_{V}^{2}+2 \int_{0}^{t}|f(s)|_{\mathrm{H}}^{2} d s\right) e^{-\lambda_{1} t+54\left(\left|u_{0}\right|_{\mathrm{H}}^{2}+\int_{0}^{t}|f(s)|_{\mathrm{V}^{\prime}}^{2} d s\right)^{2}} .
$$

so that, in particular,

$$
e^{\lambda_{1} t}|u|_{C([0, T], \mathrm{V})}^{2} \leq\left(\left|u_{0}\right|_{V}^{2}+2|f|_{L^{2}(0, T ; \mathrm{H})}^{2}\right) e^{54\left(\left|u_{0}\right|_{\mathrm{H}}^{2}+|f|_{L^{2}\left(0, T ; \mathrm{V}^{\prime}\right)}^{2}\right)^{2}} .
$$

\footnotetext{
${ }^{3}$ It is known, see for instance [42, Lemma III.1.2] that these two properties of $u$ imply that there exists a unique $\bar{u} \in C([0, T], \mathrm{H})$. When we write $u(0)$ later we mean $\bar{u}(0)$.

${ }^{4}$ Please note that $[0, T] \cap[0, \infty)$ is equal to $[0, T]$ if $T<\infty$ and to $[0, \infty)$ if $T=\infty$.
} 
Moreover, thanks to (3.4), this yields

$$
\begin{aligned}
&|u|_{L^{2}(0, T, D(\mathrm{~A})) \leq}^{2} \leq\left|u_{0}\right|_{V}^{2}+2|f|_{L^{2}(0, T ; \mathrm{H})}^{2}+54\left(\left|u_{0}\right|_{\mathrm{H}}^{2}+|f|_{L^{2}\left(0, T, \mathrm{~V}^{\prime}\right)}^{2}\right)^{2} \\
& \times\left(\left|u_{0}\right|_{V}^{2}+2|f|_{L^{2}(0, T ; \mathrm{H})}^{2}\right) e^{54\left(\left|u_{0}\right|_{\mathrm{H}}^{2}+|f|_{L^{2}\left(0, T ; \mathrm{V}^{\prime}\right)}^{2}\right)^{2}} \\
&=\left(\left|u_{0}\right|_{V}^{2}+2|f|_{L^{2}(0, T ; \mathrm{H})}^{2}\right)\left(1+54\left(\left|u_{0}\right|_{\mathrm{H}}^{2}+|f|_{L^{2}\left(0, T, \mathrm{~V}^{\prime}\right)}^{2}\right)^{2} e^{54\left(\left|u_{0}\right|_{\mathrm{H}}^{2}+|f|_{L^{2}\left(0, T ; \mathrm{V}^{\prime}\right)}^{2}\right)^{2}}\right) .
\end{aligned}
$$

The above results and arguments yield in particular the following corollary.

Corollary 3.2. If $f \in L_{\text {loc }}^{2}(0, \infty ; \mathrm{H})$ and $u_{0} \in \mathrm{V}$, then the solution $u$ to problem (3.1) satisfies $u \in L_{\mathrm{loc}}^{2}(0, \infty ; D(\mathrm{~A})), u \in C([0, \infty), \mathrm{V})$ and

$$
e^{\lambda_{1} t}|u(t)|_{V}^{2} \leq\left(\left|u_{0}\right|_{V}^{2}+2 \int_{0}^{t}|f(s)|_{\mathrm{H}}^{2} d s\right) e^{54\left(\left|u_{0}\right|_{\mathrm{H}}^{2}+\int_{0}^{t}|f(s)|_{\mathrm{V}^{\prime}}^{2} d s\right)^{2}}, \quad t \geq 0 .
$$

In particular, if $f \in L^{2}(0, \infty ; \mathrm{H})$ then $u \in L^{2}(0, \infty ; D(\mathrm{~A}))$ and

$$
e^{\lambda_{1} t}|u(t)|_{V}^{2} \leq\left(\left|u_{0}\right|_{V}^{2}+2 \int_{0}^{\infty}|f(s)|_{\mathrm{H}}^{2} d s\right) e^{54\left(\left|u_{0}\right|_{\mathrm{H}}^{2}+\int_{0}^{\infty}|f(s)|_{\mathrm{V}^{\prime}}^{2} d s\right)^{2}}, \quad t \geq 0 .
$$

If also $f=0$, this gives

$$
|u(t)|_{V}^{2} \leq e^{54\left|u_{0}\right|_{\mathrm{H}}^{4}} e^{-\lambda_{1} t}\left|u_{0}\right|_{V}^{2}, \quad t \geq 0 .
$$

Now we will formulate and prove some generalizations of the above results when the data $u_{0}$ and $f$ are slightly more regular. Similar results in the case of integer order of the Sobolev spaces has been studied in [39] where some compatibility conditions are imposed.

Proposition 3.3. Assume that $\alpha \in\left(0, \frac{1}{2}\right)$. If $T \in(0, \infty], f \in L^{2}\left(0, T ; D\left(\mathrm{~A}^{\frac{\alpha}{2}}\right)\right)$ and $u_{0} \in$ $D\left(\mathrm{~A}^{\frac{\alpha+1}{2}}\right)$, then the unique solution $u$ to problem (3.1) satisfies

$$
u \in L^{2}\left(0, T ; D\left(\mathrm{~A}^{1+\frac{\alpha}{2}}\right)\right) \cap C\left([0, T] ; D\left(\mathrm{~A}^{\frac{\alpha+1}{2}}\right)\right) \text { and } u^{\prime}(\cdot) \in L^{2}\left(0, T ; D\left(\mathrm{~A}^{\frac{\alpha}{2}}\right)\right) .
$$

Moreover, for $t \in[0, T] \cap[0, \infty)$, we have

$$
\left|\mathrm{A}^{\frac{\alpha+1}{2}} u(t)\right|_{\mathrm{H}}^{2} \leq e^{-\lambda_{1} t} e^{C^{2} K_{3}\left(\left|u_{0}\right|_{V},|f|_{L^{2}(0, T ; \mathrm{H})}\right)}\left(\left|\mathrm{A}^{\frac{\alpha+1}{2}} u_{0}\right|_{\mathrm{H}}^{2}+\int_{0}^{T}\left|\mathrm{~A}^{\frac{\alpha}{2}} f(s)\right|_{\mathrm{H}}^{2} d s\right),
$$

where $C>0$ is a generic constan and

$$
K_{3}(R, \rho):=\left[R^{2}+2 \rho^{2}\right] \times\left[1+\frac{54}{\lambda_{1}^{2}}\left(R^{2}+\rho^{2}\right)^{2} e^{\frac{54}{\lambda_{1}^{2}}\left(R^{2}+\rho^{2}\right)^{2}}\right] .
$$

In particular, if $f=0$, then

$$
\left|\mathrm{A}^{\frac{\alpha+1}{2}} u(t)\right|_{\mathrm{H}}^{2} \leq e^{-\lambda_{1} t} e^{C^{2} K_{3}\left(\left|u_{0}\right|_{V}, 0\right)}\left|\mathrm{A}^{\frac{\alpha+1}{2}} u_{0}\right|_{\mathrm{H}}^{2}, \quad t \geq 0 .
$$

\footnotetext{
${ }^{5}$ In fact, the one from inequality (2.20) in Proposition 2.5.
} 
Proof. Let us fix $T>0$. Since by Proposition 2.1. $B$ is a bilinear continuous map from $D\left(\mathrm{~A}^{\frac{\alpha+1}{2}}\right) \times D\left(\mathrm{~A}^{\frac{\alpha+1}{2}}\right)$ to $D\left(\mathrm{~A}^{\frac{\alpha}{2}}\right)$ it follows (see for instance [5] for the simplest argument) that for every $R, \rho>0$ there exists $T_{*}=T_{*}(R, \rho) \in(0, T]$ such that for every $u_{0} \in D\left(\mathrm{~A}^{\frac{\alpha+1}{2}}\right)$ and $f \in L^{2}\left(0, T ; D\left(\mathrm{~A}^{\frac{\alpha}{2}}\right)\right.$ such that

$$
\left|u_{0}\right|_{D\left(\mathrm{~A}^{\frac{\alpha+1}{2}}\right)} \leq R, \quad|f|_{L^{2}\left(0, T_{*} ; D\left(\mathrm{~A}^{\frac{\alpha}{2}}\right)\right.} \leq \rho
$$

there exists a unique solution $v$ to problem (3.1) which satisfy conditions (3.11) on the time interval $\left[0, T_{*}\right]$. Since $D\left(\mathrm{~A}^{\frac{\alpha+1}{2}}\right) \subset \mathrm{V}$ and $L^{2}\left(0, T ; D\left(\mathrm{~A}^{\frac{\alpha}{2}}\right) \subset L^{2}(0, T ; \mathrm{H})\right.$ with the embeddings being continuous, $u_{0} \in \mathrm{V}$ and $f \in L^{2}(0, T ; \mathrm{H})$. Therefore by Theorems 3.1 and 3.2 in chapter III of [42, there exists a unique solution $u$ to problem (3.1) on the whole real half-line $[0, \infty)$ which satisfies (3.6) and (3.7). By the uniqueness part of the above cited results, $u=v$ on $\left[0, T_{*}\right]$.

Hence it is sufficient to show that the norm of $u$ in $L^{2}\left(0, T_{*} ; D\left(\mathrm{~A}^{1+\frac{\alpha}{2}}\right) \cap C\left(\left[0, T_{*}\right] ; D\left(\mathrm{~A}^{\frac{\alpha+1}{2}}\right)\right)\right.$ and the norm of $u^{\prime}$ in $L^{2}\left(0, T_{*} ; D\left(\mathrm{~A}^{\frac{\alpha}{2}}\right)\right)$ are bounded by a constant depending only on $\left|\mathrm{A}^{\frac{\alpha+1}{2}} u_{0}\right|_{\mathrm{H}}^{2}$ and $\int_{0}^{T}\left|\mathrm{~A}^{\frac{\alpha}{2}} f(s)\right|_{\mathrm{H}}^{2} d s$.

For this aim, by calculating the derivative of $\left|\mathrm{A}^{\frac{\alpha+1}{2}} u(t)\right|_{\mathrm{H}}^{2}$, i.e. applying Lemma III.1.2 from 42 and using inequality (2.20), with $s=2$, we get the following inequality

$$
\begin{aligned}
& \frac{1}{2} \frac{d}{d t}\left|\mathrm{~A}^{\frac{\alpha+1}{2}} u(t)\right|_{\mathrm{H}}^{2}+\left|\mathrm{A}^{\frac{\alpha}{2}+1} u(t)\right|_{\mathrm{H}}^{2} \\
& \quad \leq\left|\mathrm{A}^{\frac{\alpha}{2}} f(t)\right|_{\mathrm{H}}^{2}+C^{2}|\mathrm{~A} u(t)|_{\mathrm{H}}^{2}\left|\mathrm{~A}^{\frac{\alpha+1}{2}} u(t)\right|_{\mathrm{H}}^{2}, \quad t \in\left[0, T_{*}\right] .
\end{aligned}
$$

Thus, denoting the right hand side of inequality (3.7) by $K_{1}\left(T, f, u_{0}\right)$ and applying the Gronwall Lemma we get

$$
\begin{aligned}
\mid \mathrm{A}^{\frac{\alpha+1}{2}} & \left.u(t)\right|_{\mathrm{H}} ^{2}+\int_{0}^{t}\left|\mathrm{~A}^{\frac{\alpha}{2}+1} u(s)\right|_{\mathrm{H}}^{2} d s \\
& \leq e^{C^{2} K_{1}\left(T, f, u_{0}\right)}\left|\mathrm{A}^{\frac{\alpha+1}{2}} u_{0}\right|_{\mathrm{H}}^{2}+e^{C^{2} K_{1}\left(T, f, u_{0}\right)} \int_{0}^{t}\left|\mathrm{~A}^{\frac{\alpha}{2}} f(s)\right|_{\mathrm{H}}^{2} d s \\
& \leq e^{C^{2} K_{1}\left(T, f, u_{0}\right)}\left(\left|\mathrm{A}^{\frac{\alpha+1}{2}} u_{0}\right|_{\mathrm{H}}^{2}+\int_{0}^{T}\left|\mathrm{~A}^{\frac{\alpha}{2}} f(s)\right|_{\mathrm{H}}^{2} d s\right), \quad t \in\left[0, T_{*}\right] .
\end{aligned}
$$

This proves that the norm of $u$ in $L^{2}\left(0, T_{*} ; D\left(\mathrm{~A}^{1+\frac{\alpha}{2}}\right) \cap C\left(\left[0, T_{*}\right] ; D\left(\mathrm{~A}^{\frac{\alpha+1}{2}}\right)\right)\right.$ is bounded by a constant depending only on $\left|\mathrm{A}^{\frac{\alpha+1}{2}} u_{0}\right|_{\mathrm{H}}^{2}$ and $\int_{0}^{T}\left|\mathrm{~A}^{\frac{\alpha}{2}} f(s)\right|_{\mathrm{H}}^{2} d s$.

Finally, the corresponding bound for the norm of $u^{\prime}$ in $L^{2}\left(0, T_{*} ; D\left(\mathrm{~A}^{\frac{\alpha}{2}}\right)\right)$ follows from estimate (3.4), inequality (2.20) from Proposition 2.5, the assumption on $f$ and the estimates for $\mathrm{A} u$ and $\mathrm{B}(u, u)$.

\footnotetext{
${ }^{6}$ Since $\left|\left\langle\mathrm{A}^{1+\alpha} u, B u\right\rangle\right|=\left|\left\langle\mathrm{A}^{1+\frac{\alpha}{2}} u, \mathrm{~A}^{\frac{\alpha}{2}} B u\right\rangle\right| \leq\left|\mathrm{A}^{1+\frac{\alpha}{2}} u\right|\left|\mathrm{A}^{\frac{\alpha}{2}} B u\right| \leq C\left|\mathrm{~A}^{1+\frac{\alpha}{2}} u\|\mathrm{~A} u\| \mathrm{A}^{\frac{1+\alpha}{2}} u\right| \leq \frac{1}{4}\left|\mathrm{~A}^{1+\frac{\alpha}{2}} u\right|^{2}+$ $C^{2}|\mathrm{~A} u|^{2}\left|\mathrm{~A}^{\frac{1+\alpha}{2}} u\right|^{2}$.
} 
This concludes the proof of the first part of Proposition 3.3, in particular of (3.11).

Let us now assume that $T=\infty, R, \rho>0$ and $f \in L^{2}\left(0, \infty ; D\left(\mathrm{~A}^{\frac{\alpha}{2}}\right)\right)$ and $u_{0} \in D\left(\mathrm{~A}^{\frac{\alpha+1}{2}}\right)$ such that $\left|u_{0}\right|_{V} \leq R$ and $|f|_{L^{2}(0, \infty ; \mathrm{H})} \leq \rho$. Since, by the Poincaré inequality (2.1),$|f|_{\mathrm{V}^{\prime}}^{2} \leq \lambda_{1}^{-1}|f|_{\mathrm{H}}^{2}$, for $f \in \mathrm{H}$, we infer that $K_{1}\left(T, f, u_{0}\right) \leq K_{3}(R, \rho)$, where $K_{3}(R, \rho)$ has been defined in (3.13). Thus, from inequality (3.15) we infer

$$
\left|\mathrm{A}^{\frac{\alpha+1}{2}} u(t)\right|_{\mathrm{H}}^{2}+\int_{0}^{t}\left|\mathrm{~A}^{\frac{\alpha}{2}+1} u(s)\right|_{\mathrm{H}}^{2} d s \leq e^{C^{2} K_{3}(R, \rho)}\left(\left|\mathrm{A}^{\frac{\alpha+1}{2}} u_{0}\right|_{\mathrm{H}}^{2}+\int_{0}^{t}\left|\mathrm{~A}^{\frac{\alpha}{2}} f(s)\right|_{\mathrm{H}}^{2} d s\right), \quad t \geq 0 .
$$

Since by the Poincaré inequality (2.1) $\left|\mathrm{A}^{\frac{\alpha}{2}+1}\right|_{\mathrm{H}}^{2} \geq \lambda_{1}\left|\mathrm{~A}^{\frac{\alpha+1}{2}} u\right|_{\mathrm{H}}^{2}$, for $u \in D\left(\mathrm{~A}^{\frac{\alpha}{2}+1}\right)$, by the inequality above and the Gronwall Lemma we infer that

$$
\left|\mathrm{A}^{\frac{\alpha+1}{2}} u(t)\right|_{\mathrm{H}}^{2} \leq e^{-\lambda_{1} t} e^{C^{2} K(R, \rho)}\left(\left|\mathrm{A}^{\frac{\alpha+1}{2}} u_{0}\right|_{\mathrm{H}}^{2}+\int_{0}^{t}\left|\mathrm{~A}^{\frac{\alpha}{2}} f(s)\right|_{\mathrm{H}}^{2} d s\right), \quad t \geq 0 .
$$

This concludes the proof of inequality (3.12) and hence of the second part of the Proposition.

The previous result can be used to derive the next corollary. The proof of this corollary is analogous to the proof of properties (3.151) in Theorem 3.10 from [42, chapter III].

Corollary 3.4. Assume that $\alpha \in\left(0, \frac{1}{2}\right)$ and $\beta \in(\alpha, 1)$. If $T \in(0, \infty), f \in L^{2}\left(0, T ; D\left(\mathrm{~A}^{\frac{\alpha}{2}}\right)\right)$ and $u_{0} \in \mathrm{V}$, then the unique solution $u$ to the problem (3.1) satisfy

$$
\begin{gathered}
\sup _{s \in(0, T]} s^{\beta}\left|\mathrm{A}^{\frac{\alpha+1}{2}} u(s)\right|_{\mathrm{H}}^{2}+\int_{0}^{T} s^{\beta}\left|\mathrm{A}^{\frac{\alpha}{2}+1} u(s)\right|_{\mathrm{H}}^{2} d s \leq e^{C^{2} K_{1}\left(T, f, u_{0}\right)}\left[\int_{0}^{T} s^{\beta}\left|\mathrm{A}^{\frac{\alpha}{2}} f(s)\right|_{\mathrm{H}}^{2} d s\right. \\
\left.+C T^{\frac{1}{\beta-\alpha}}\left(K_{0}\left(T, f, u_{0}\right)+K_{1}\left(T, f, u_{0}\right)\right)\right] .
\end{gathered}
$$

Proof. Let us fix $\alpha \in\left(1, \frac{1}{2}\right), \beta \in(\alpha, 1), f \in L^{2}\left(0, T ; D\left(\mathrm{~A}^{\frac{\alpha}{2}}\right)\right)$ and $u_{0} \in D\left(\mathrm{~A}^{\frac{\alpha+1}{2}}\right)$. Multiplying the differential inequality $(3.15)$ by $t^{\beta}$ and then integrating it, we get

$$
\begin{aligned}
t^{\beta}\left|\mathrm{A}^{\frac{\alpha+1}{2}} u(t)\right|_{\mathrm{H}}^{2}+\int_{0}^{t} s^{\beta}\left|\mathrm{A}^{\frac{\alpha}{2}+1} u(s)\right|_{\mathrm{H}}^{2} d s & \leq \int_{0}^{t} s^{\beta}\left|\mathrm{A}^{\frac{\alpha}{2}} f(s)\right|_{\mathrm{H}}^{2} d s \\
+C^{2} \int_{0}^{t}|\mathrm{~A} u(s)|_{\mathrm{H}}^{2} s^{\beta}\left|\mathrm{A}^{\frac{\alpha+1}{2}} u(s)\right|_{\mathrm{H}}^{2} d s & +\beta \int_{0}^{t} s^{\beta-1}\left|\mathrm{~A}^{\frac{\alpha+1}{2}} u(s)\right|_{\mathrm{H}}^{2} d s, \quad t \in(0, T] .
\end{aligned}
$$

Since $A$ is a self-adjoint operator in $\mathrm{H}$ we have

$$
\left|\mathrm{A}^{\frac{\alpha+1}{2}} u\right|_{\mathrm{H}}^{2} \leq\left|\mathrm{A}^{\frac{1}{2}} u\right|_{\mathrm{H}}^{2(1-\alpha)}\left|\mathrm{A}^{\frac{2}{2}} u\right|_{\mathrm{H}}^{2 \alpha}=|u|_{V}^{2(1-\alpha)}|\mathrm{A} u|_{\mathrm{H}}^{2 \alpha}, \quad u \in D(\mathrm{~A}) .
$$

Therefore, by the Hölder inequality,

$$
\int_{0}^{t} s^{\beta-1}\left|\mathrm{~A}^{\frac{\alpha+1}{2}} u(s)\right|_{\mathrm{H}}^{2} d s \leq\left(\sup _{s \in(0, t]}|u(s)|_{V}^{2} \int_{0}^{t} s^{\frac{\beta-1}{1-\alpha}} d s\right)^{1-\alpha}\left(\int_{0}^{t}|\mathrm{~A} u(s)|^{2} d s\right)^{\alpha} .
$$


Since we are assuming $\beta>\alpha$ and $\alpha<1$, we infer that $\frac{\beta-1}{1-\alpha}>-1$ and therefore

$$
\int_{0}^{t} s^{\frac{\beta-1}{1-\alpha}} d s=\frac{1-\alpha}{\beta-\alpha} t^{\frac{1-\alpha}{\beta-\alpha}}<\infty .
$$

Let us recall that by $K_{1}\left(T, f, u_{0}\right)$ we denote the right hand side of inequality (3.7). Let us also denote by $K_{0}\left(T, f, u_{0}\right)$ the right hand side of inequality (3.6). Then, for a constant $C$ depending only on $\alpha$ and $\beta$ we get

$$
\beta \int_{0}^{T} s^{\beta-1}\left|\mathrm{~A}^{\frac{\alpha+1}{2}} u(s)\right|_{\mathrm{H}}^{2} d s \leq C T^{\frac{1}{\beta-\alpha}} K_{0}\left(T, f, u_{0}\right)^{1-\alpha} K_{1}\left(T, f, u_{0}\right)^{\alpha}<\infty .
$$

Therefore, we can deduce from inequality (3.18) the following one

$$
\begin{aligned}
t^{\beta}\left|\mathrm{A}^{\frac{\alpha+1}{2}} u(t)\right|_{\mathrm{H}}^{2}+\int_{0}^{t} s^{\beta}\left|\mathrm{A}^{\frac{\alpha}{2}+1} u(s)\right|_{\mathrm{H}}^{2} d s & \leq e^{C^{2} K_{1}\left(T, f, u_{0}\right)}\left(\int_{0}^{t} s^{\beta}\left|\mathrm{A}^{\frac{\alpha}{2}} f(s)\right|_{\mathrm{H}}^{2} d s\right. \\
& \left.+C t^{\frac{1}{\beta-\alpha}} K_{0}\left(t, f, u_{0}\right)^{1-\alpha} K\left(t, f, u_{0}\right)^{\alpha}\right) \\
\leq e^{C^{2} K_{1}\left(T, f, u_{0}\right)}\left(\int_{0}^{T} s^{\beta}\left|\mathrm{A}^{\frac{\alpha}{2}} f(s)\right|_{\mathrm{H}}^{2} d s\right. & \left.+C T^{\frac{1}{\beta-\alpha}}\left(K_{0}\left(T, f, u_{0}\right)+K_{1}\left(T, f, u_{0}\right)\right)\right), \quad t \in[0, T] .
\end{aligned}
$$

This implies inequality (3.17) under the additional assumption that $u_{0} \in D\left(\mathrm{~A}^{\frac{\alpha+1}{2}}\right)$.

Now, if $u_{0} \in \mathrm{V}$, then by [42, Theorem III.3.10], see also inequality (3.7), there exists a sequence $\left\{t_{n}\right\}$ such $t_{n} \searrow 0$ and $u\left(t_{n}\right) \in D(\mathrm{~A}) \subset D\left(\mathrm{~A}^{\frac{\alpha+1}{2}}\right)$. Let us also denote $f_{n}=f_{\mid\left[t_{n}, T\right]}$ and observe that $K_{i}\left(T-t_{n}, f_{n}, u\left(t_{n}\right)\right) \leq K_{i}\left(T, f, u\left(t_{n}\right)\right)$. Thus, by applying inequality (3.17) to our solution $u$ on the time interval $\left[t_{n}, T\right]$ we get for each $n \in \mathbb{N}$

$$
\begin{aligned}
& \sup _{s \in\left(t_{n}, T\right]}\left(s-t_{n}\right)^{\beta}\left|\mathrm{A}^{\frac{\alpha+1}{2}} u(s)\right|_{\mathrm{H}}^{2}+\int_{t_{n}}^{T}\left(s-t_{n}\right)^{\beta}\left|\mathrm{A}^{\frac{\alpha}{2}+1} u(s)\right|_{\mathrm{H}}^{2} d s \leq e^{K_{1}\left(T, f, u\left(t_{n}\right)\right)} \\
& \times\left(\int_{t_{n}}^{T}\left(s-t_{n}\right)^{\beta}\left|\mathrm{A}^{\frac{\alpha}{2}} f(s)\right|_{\mathrm{H}}^{2} d s+C\left(T-\left(t_{n}\right)\right)^{\frac{1}{\beta-\alpha}}\left(K_{0}\left(T, f, u\left(t_{n}\right)\right)+K_{1}\left(T, f, u\left(t_{n}\right)\right)\right)\right) .
\end{aligned}
$$

Since $u \in C([0, T] ; V)$, we infer that $\left|u\left(t_{n}\right)\right|_{V} \rightarrow\left|u_{0}\right|_{V}$ and thus $K_{i}\left(T, f, u\left(t_{n}\right)\right) \rightarrow K_{i}\left(T, f, u_{0}\right)$, $i=1,2$. Moreover, by the Lebesgue Monotone Convergence Theorem,

$$
\begin{aligned}
\int_{t_{n}}^{T}\left(s-t_{n}\right)^{\beta}\left|\mathrm{A}^{\frac{\alpha}{2}+1} u(s)\right|_{\mathrm{H}}^{2} d s & =\int_{0}^{T} 1_{\left(t_{n}, T\right]}(s)\left(s-t_{n}\right)^{\beta}\left|\mathrm{A}^{\frac{\alpha}{2}+1} u(s)\right|_{\mathrm{H}}^{2} d s \rightarrow \int_{0}^{T} s^{\beta}\left|\mathrm{A}^{\frac{\alpha}{2}+1} u(s)\right|_{\mathrm{H}}^{2} d s, \\
\int_{t_{n}}^{T}\left(s-t_{n}\right)^{\beta}\left|\mathrm{A}^{\frac{\alpha}{2}} f(s)\right|_{\mathrm{H}}^{2} d s & =\int_{0}^{T} 1_{\left(t_{n}, T\right]}(s)\left(s-t_{n}\right)^{\beta}\left|\mathrm{A}^{\frac{\alpha}{2}} f(s)\right|_{\mathrm{H}}^{2} d s \rightarrow \int_{0}^{T} s^{\beta}\left|\mathrm{A}^{\frac{\alpha}{2}} f(s)\right|_{\mathrm{H}}^{2} d s .
\end{aligned}
$$

Hence, from (3.21) we deduce (3.17). The proof is complete. 
Now, for any $-\infty \leq a<b \leq \infty$ such that $a<b$ and for any two reflexive Banach spaces $X$ and $Y$ such that $X \hookrightarrow \bar{Y}$ continuously, we denote by $W^{1,2}(a, b ; X, Y)$ the space of all $u \in L^{2}(a, b ; X)$ which are weakly differentiable as $Y$-valued functions and their weak derivative belongs to $L^{2}(a, b ; Y)$. The space $W^{1,2}(a, b ; X, Y)$ is a separable Banach space (and Hilbert if both $X$ and $Y$ are Hilbert spaces), with the natural norm

$$
|u|_{W^{1,2}(a, b ; X, Y)}^{2}=|u|_{L^{2}(a, b ; X)}^{2}+\left|u^{\prime}\right|_{L^{2}(a, b ; Y)}^{2}, \quad u \in W^{1,2}(a, b ; X, Y) .
$$

Later on, we will use the shortcut notation

$$
W^{1,2}(a, b)=W^{1,2}(a, b ; D(\mathrm{~A}), \mathrm{H}) .
$$

We conclude this section with the statement of a couple of results which are obvious adaptations of deep results from [31] to the 2-dimensional case. To this purpose, there is no need to mention that all what we have said about equation (3.1) in the time interval $[0, T]$ applies to any time interval $[a, b]$, with $-\infty<a<b<\infty$.

Definition 3.5. Assume that $-\infty \leq a<b \leq \infty$ and $f \in L_{l o c}^{2}((a, b) ; \mathrm{H})$. A function $u \in$ $C((a, b) ; \mathrm{H})$ is called a very weak solution to the Navier-Stokes equations (3.1) on the interval $(a, b)$ if for all $\phi \in C^{\infty}((a, b) \times D)$, such that $\operatorname{div} \phi=0$ on $(a, b) \times D$ and $\phi=0$ on $(a, b) \times \partial D$,

$$
\begin{aligned}
& \int_{D} u\left(t_{1}, x\right) \phi\left(t_{1}, x\right) d x \\
& =\int_{D} u\left(t_{0}, x\right) \phi\left(t_{0}, x\right) d x+\int_{\left[t_{0}, t_{1}\right] \times D} u(s, x) \cdot\left(\partial_{s} \phi(s, x)+\nu \Delta \phi(s, x)\right) d s d x \\
& +\int_{t_{0}}^{t_{1}} b(u(s), u(s), \phi(s)) d s+\int_{\left[t_{0}, t_{1}\right] \times D} f(s, x) \cdot \phi(s, x) d s d x
\end{aligned}
$$

for all $a<t_{0}<t_{1}<b$.

Proposition 3.6. Assume that $-\infty \leq a<b \leq \infty$ and $f \in L_{l o c}^{2}((a, b) ; \mathrm{H})$. Suppose that the functions $u, v \in C((a, b) ; \mathrm{H})$ are very weak solutions to the Navier-Stokes equations (3.1) on the interval $(a, b)$, with $u\left(t_{0}\right)=v\left(t_{0}\right)$, for some $t_{0} \in(a, b)$. Then $u(t)=v(t)$ for all $t \geq t_{0}$.

In the whole paper we will assume, without any loss of generality, that $\nu=1$.

Definition 3.7. Assume that $-\infty \leq a<b \leq \infty$. Given a function $u \in C((a, b) ; \mathrm{H})$ we say that

$$
u^{\prime}+\mathrm{A} u+\mathrm{B}(u, u) \in L^{2}(a, b ; \mathrm{H}), \quad\left(\text { resp. } \in L_{l o c}^{2}((a, b) ; \mathrm{H})\right)
$$

if there exists $f \in L^{2}(a, b ; \mathrm{H})$, (resp. $f \in L_{\text {loc }}^{2}((a, b) ; \mathrm{H})$ ) such that $u$ is a very weak solution of the Navier-Stokes equations (3.1) on the interval $(a, b)$.

Clearly, the corresponding function $f$ is unique and we will denote it by $\mathcal{H}(u)$, i.e.

$$
[\mathcal{H}(u)]:=u^{\prime}+\mathrm{A} u+\mathrm{B}(u, u)
$$

\footnotetext{
${ }^{7}$ Some authours, for instance Vishik and Fursikov, use the notation $\mathcal{H}^{1,2} N(a, b ; X, Y)$. Our choice is motivated by the notation used in the monograph [30, who however use notation $W(a, b)$.
} 
An obvious sufficient condition for the finiteness of the norm of $\mathcal{H}(u)$ in $L^{2}\left(t_{0}, t_{1} ; H\right)$ is that $u^{\prime}, A u$ and $B(u, u)$ all belong to $L^{2}\left(t_{0}, t_{1} ; \mathrm{H}\right)$. The next result shows that this is not so far from a necessary condition. This is the reason why we have decided present the following result based [31, see also [25].

Lemma 3.8. Suppose that $T>0$ and $u \in C([0, T] ; \mathrm{H})$ is such that

$$
u^{\prime}+\mathrm{A} u+\mathrm{B}(u, u) \in L^{2}(0, T ; \mathrm{H}) .
$$

Then $u(T) \in \mathrm{V}, u \in W^{1,2}\left(t_{1}, T\right)$, for any $t_{1} \in(0, T)$ and

$$
\sup _{t \in(0, T]}|\sqrt{t} u(t)|_{V}^{2}+\int_{0}^{T}|\sqrt{t} \mathrm{~A} u(t)|_{\mathrm{H}}^{2} d t<\infty .
$$

Moreover, if $u(0) \in \mathrm{V}$, then $u \in W^{1,2}(0, T)$.

Proof. Let us fix $T>0$ and $u$ as in the assumptions of the Lemma and let us denote $f=u^{\prime}+\mathrm{A} u+\mathrm{B}(u, u)$. By assumptions we infer that $f \in L^{2}(0, T ; \mathrm{H})$. Since $u(0) \in \mathrm{H}$, by [42, Theorem III.3.10], there exists a unique solution $v$ to problem (3.1) which satisfies inequality (3.3). Then by $v$ is also a mild solution to (3.1) and since by assumptions and 31 , Proposition 2.5] $u \in C([0, T] ; \mathrm{H})$ is also a mild solution to (3.1), by Proposition 3.6 (i.e. 31, Theorem 1.2]) we infer that $u=v$. Hence $u \in L^{2}(0, T ; \mathrm{V}), u^{\prime} \in L^{2}\left(0, T ; \mathrm{V}^{\prime}\right)$ and $u$ satisfies (3.3). In particular, for every $t_{1} \in(0, T)$ we can find $t_{0} \in\left(0, t_{1}\right)$ such that $u\left(t_{0}\right) \in \mathrm{V}$ and therefore by [42, Theorem III.3.10], $u \in W^{1,2}\left(t_{0}, T\right)$. In particular, $u \in C\left(\left[t_{0}, T\right] ; \mathrm{V}\right)$ and hence $u(T) \in \mathrm{V}$.

If the additional assumption that $u(0) \in \mathrm{V}$ is satisfied, then by what we have just seen ([42, Theorem III.3.10]) or by the maximal regularity and the uniqueness of solutions to 2D NSEs), we can conclude that $u \in W^{1,2}(0, T)$.

Remark 1. It should be pointed out that we cannot claim that $u(0) \in \mathrm{V}$. Indeed, if we take a solution $u$ of the problem (3.1) with data $u_{0} \in \mathrm{H} \backslash \mathrm{V}$ and $f=0$, then $u$ satisfies the Assumptions of Lemma 3.8 but nethervelles $u(0) \notin V$. See however Proposition A.1 for a positive result on an unbounded interval $(-\infty, 0]$.

A result analogous of Lemma 3.8 holds in domains of fractional powers of A.

Lemma 3.9. Assume that $\alpha \in[0,1 / 2)$ and suppose that $u \in C([0, T] ; \mathrm{H})$, for some $T>0$, is such that

$$
u^{\prime}+\mathrm{A} u+\mathrm{B}(u, u) \in L^{2}\left(0, T ; D\left(\mathrm{~A}^{\frac{\alpha}{2}}\right)\right) .
$$

Then $u(T) \in D\left(\mathrm{~A}^{\frac{\alpha+1}{2}}\right)$ and $u \in W^{1,2}\left(t_{0}, T ; D\left(\mathrm{~A}^{\frac{\alpha}{2}+1}\right), D\left(\mathrm{~A}^{\frac{\alpha}{2}}\right)\right)$, for any $t_{0} \in(0, T)$. Moreover, if $u(0) \in D\left(\mathrm{~A}^{\frac{\alpha+1}{2}}\right)$, then $u \in W^{1,2}\left(0, T ; D\left(\mathrm{~A}^{\frac{\alpha}{2}+1}\right), D\left(\mathrm{~A}^{\frac{\alpha}{2}}\right)\right)$.

Proof. Denote $f=u^{\prime}+\mathrm{A} u+\mathrm{B}(u, u)$ and let us fix $t_{0} \in(0, T)$ and some $t_{1} \in\left(0, t_{0}\right)$. By Lemma 3.8 we infer that $u \in W^{1,2}\left(t_{1}, T\right)$. In particular, there exists $t_{2} \in\left(t_{1}, t_{0}\right)$ such that $u\left(t_{2}\right) \in D(\mathrm{~A}) \subset D\left(\mathrm{~A}^{\frac{\alpha+1}{2}}\right)$. The last embedding holds since by assumptions $\alpha<\frac{1}{2}$. Since by our assumption, $f \in L^{2}\left(0, T ; D\left(\mathrm{~A}^{\frac{\alpha}{2}}\right)\right)$, in view of Proposition 3.3 and Proposition 3.6 , 
we infer that $u \in W^{1,2}\left(t_{2}, T ; D\left(\mathrm{~A}^{\frac{\alpha}{2}+1}\right), D\left(\mathrm{~A}^{\frac{\alpha}{2}}\right)\right)$. This implies that $u \in C\left(\left[t_{2}, T\right] ; D\left(\mathrm{~A}^{\frac{\alpha+1}{2}}\right)\right)$ and in particular that $u(T) \in D\left(\mathrm{~A}^{\frac{\alpha+1}{2}}\right)$ and $u \in W^{1,2}\left(t_{0}, T ; D\left(\mathrm{~A}^{\frac{\alpha}{2}+1}\right), D\left(\mathrm{~A}^{\frac{\alpha}{2}}\right)\right)$ as in our first claim. The second claim follows from our last argument.

In what follows, for any $r>0$ and $\gamma \geq 0$ we shall denote by $B_{\gamma}(r)$ the closed ball in $D\left(\mathrm{~A}^{\frac{\gamma}{2}}\right)$ of radius $r$ and centered at the origin, i.e.

$$
B_{\gamma}(r):=\left\{x \in D\left(\mathrm{~A}^{\frac{\gamma}{2}}\right):|x|_{D\left(\mathrm{~A}^{\frac{\gamma}{2}}\right)} \leq r\right\} .
$$

Moreover, for any $\phi \in \mathrm{H}$ and $s \in \mathbb{R}$, we shall denote by $u_{\phi}(t ; s), t \geq s$ (simply $u_{\phi}(t), t \geq 0$, when $s=0$ ) the solution of problem (3.1) with the external force $f$ equal to 0 , i.e.

$$
\left\{\begin{array}{l}
u^{\prime}(t)+\mathrm{A} u(t)+\mathrm{B}(u(t), u(t))=0, \quad t>s, \\
u(s)=\phi .
\end{array}\right.
$$

Moreover, for $\phi \in \mathrm{H}, r>0$ and $\gamma \geq 0$ we shall denote

$$
t_{\phi}^{r, \gamma}:=\inf \left\{t \geq 0: u_{\phi}(t) \in B_{\gamma}(r)\right\} .
$$

Proposition 3.10. For any $c_{1}, c_{2}>0$ and $\sigma \in\left[0, \frac{3}{2}\right)$, there exists $T=T\left(\sigma, c_{1}, c_{2}\right)>0$ such that for every $\phi \in \mathrm{H}$ such $|\phi|_{\mathrm{H}} \leq c_{1}$, one has

$$
\left|A^{\frac{\sigma}{2}} u_{\phi}(t)\right|_{\mathrm{H}} \leq c_{2}, \text { for all } t \geq T \text {. }
$$

Proof. By inequality (3.3), for any $\phi \in \mathrm{H}$

$$
\left|u_{\phi}(1)\right|_{V}^{2} \leq|\phi|_{\mathrm{H}}^{2} e^{C|\phi|_{\mathrm{H}}^{4}} .
$$

Then, by inequality (3.10) in Corollary 3.2, we infer

$$
\left|u_{\phi}(t)\right|_{V}^{2} \leq e^{54|\phi|_{H}^{4}} e^{-\lambda_{1}(t-1)}\left|u_{\phi}(1)\right|_{V}^{2}, \quad t \geq 1 .
$$

Combining these two we get

$$
\left|u_{\phi}(t)\right|_{V}^{2} \leq e^{(54+C)|\phi|_{\mathrm{H}}^{4}+\lambda_{1}} e^{-\lambda_{1} t}|\phi|_{\mathrm{H}}^{2}, \quad t \geq 1
$$

and (3.25) follows for $\sigma \leq 1$.

Consider now the case $\sigma \in\left(1, \frac{3}{2}\right)$. Let us fix constants $c_{1}, c_{2}>0$ and an initial data $\phi \in \mathrm{H}$ such that $|\phi|_{\mathrm{H}} \leq c_{1}$. We will be applying the previous step with $\alpha=\sigma-1 \in\left(0, \frac{1}{2}\right)$. Choose an auxiliary $\beta \in(\sigma-1,1)$. Then by inequality (3.17) in Corollary 3.4, we get

$$
\left|\mathrm{A}^{\frac{\alpha+1}{2}} u_{\phi}(2)\right|^{2} \leq e^{C^{2} K_{1}\left(2,0, u_{\phi}(1)\right)} C\left(K_{0}\left(2,0, u_{\phi}(1)\right)+K_{1}\left(2,0, u_{\phi}(1)\right)\right) .
$$

Therefore, recalling how $K_{0}$ and $K_{1}$ were defined, due to (3.26)

$$
\left|\mathrm{A}^{\frac{1+\alpha}{2}} u_{\phi}(2)\right|_{\mathrm{H}}^{2} \leq K_{4}\left(|\phi|_{\mathrm{H}}\right),
$$

for some continuous, increasing function $K_{4}$. According to (3.12) this allows to conclude. 


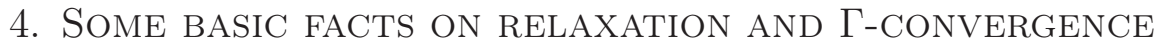

Let us assume that $X$ is a topological space satisfying the first axiom of countability, i.e. every point in $X$ has a countable local base. For any $x \in X$, we shall denote by $\mathcal{N}(x)$ the set of all open neighborhoods of $x$ in $X$.

Definition 4.1. Let $F: X \rightarrow \overline{\mathbb{R}}$ be a function.

(1) The function $F$ is called lower semi-continuous if for any $t \in \mathbb{R}$, the inverse image set $F^{-1}((-\infty])=\{x \in X: F(x) \leq t\}$ is closed in $X$,

(2) The function $F$ is called coercive if for any $t \in \mathbb{R}$, the closure of the level set $\{x \in X$ : $F(x) \leq t\}$ is countably compact, i.e. every countable open cover has a finite subcover.

Now, let $\left\{F_{n}\right\} \in \mathbb{N}$ be a sequence of functions all defined on $X$ with values in $\overline{\mathbb{R}}$.

Definition 4.2. The sequence of functions $\left\{F_{n}\right\}_{\in \mathbb{N}}$ is called equi-coercive if for any $t \in \mathbb{R}$ there exists a closed countably compact set $K_{t} \subset X$ such that

$$
\bigcup_{n \in \mathbb{N}}\left\{x \in X: F_{n}(x) \leq t\right\} \subset K_{t} .
$$

Let us note that if $Y$ is a closed subspace of $X$, then the restrictions to $Y$ of lower semicontinuous, coercive and equi-coercive functions, remain such on $Y$.

As proved in [15, Proposition 7.7], the following characterization of equi-coercive sequences holds.

Proposition 4.3. The sequence $\left\{F_{n}\right\} \in \mathbb{N}$ is equi-coercive if and only if there exists a lower semi-continuous coercive function $\Psi: X \rightarrow \overline{\mathbb{R}}$ such that

$$
F_{n}(x) \geq \Psi(x), \quad x \in X, \quad n \in \mathbb{N} .
$$

Now, we introduce the notion of relaxation of a function $F$.

Definition 4.4. The lower semi-continuous envelope, or the relaxed function, of a function $F: X \rightarrow \overline{\mathbb{R}}$ is defined by

$$
\left(s c^{-} F\right)(x)=\sup \{G(x): G \in \mathcal{G}(F)\}, \quad x \in X,
$$

where $\mathcal{G}(F)$ is the set of all lower semi-continuous functions $G: X \rightarrow \overline{\mathbb{R}}$ such that $G \leq F$.

From the definition, one has immediately that $s c^{-} F$ is lower semi-continuous, $s c^{-} F \leq F$ and $s c^{-} F \geq G$, for any $G \in \mathcal{G}(F)$, so that $s c^{-} F$ can be regarded as the greatest lower semi-continuous function majorized by $F$. Moreover, it is possible to prove that

$$
\left(s c^{-} F\right)(x)=\sup _{U \in \mathcal{N}(x)} \inf _{y \in U} F(y), \quad x \in X,
$$

(see [15, Proposition 3.3]).

The following result, whose proof can be found in [15, Proposition 3.6], provides a possible characterization of $s c^{-} F$ which we will use later on in the paper.

Proposition 4.5. For any function $F: X \rightarrow \overline{\mathbb{R}}$, its lower semi-continuous function sc $F$ is characterized by the following properties:

(1) for any $x \in X$ and any sequence $\left\{x_{n}\right\}_{n \in \mathbb{N}}$ convergent to $x$ in $X$, it holds

$$
\left(s c^{-} F\right)(x) \leq \liminf _{n \rightarrow \infty} F\left(x_{n}\right)
$$


(2) for any $x \in X$ there exists a sequence $\left\{x_{n}\right\}_{n \in \mathbb{N}}$ convergent to $x$ in $X$ such that

$$
\left(s c^{-} F\right)(x) \geq \limsup _{n \rightarrow \infty} F\left(x_{n}\right) .
$$

Next, we introduce the notion of $\Gamma$-convergence for sequences of functions.

Definition 4.6. The $\Gamma$-lower limit and the $\Gamma$-upper limit of the sequence $\left\{F_{n}\right\}_{n \in \mathbb{N}}$ are the functions from $X$ into $\overline{\mathbb{R}}$ defined respectively by

$$
\begin{aligned}
& \Gamma-\liminf _{n \rightarrow \infty} F_{n}(x)=\sup _{U \in \mathcal{N}(x)} \liminf _{n \rightarrow \infty} \inf _{y \in U} F_{n}(y), \\
& \Gamma-\limsup _{n \rightarrow \infty} F_{n}(x)=\sup _{U \in \mathcal{N}(x)} \limsup _{n \rightarrow \infty} \inf _{y \in U} F_{n}(y) .
\end{aligned}
$$

If there exists a function $F: X \rightarrow \overline{\mathbb{R}}$ such that $\Gamma-\liminf _{n \rightarrow \infty} F_{n}=\Gamma-\lim _{\sup _{n \rightarrow \infty}} F_{n}=F$, then we write

$$
F=\Gamma-\lim _{n \rightarrow \infty} F_{n},
$$

and we say that the sequence $\left\{F_{n}\right\}_{n \in \mathbb{N}}$ is $\Gamma$-convergent to $F$.

In [15, Proposition 5.7] we can find the proof of the following result, which links $\Gamma$-convergence and relaxation of functions and provides a useful criterium for $\Gamma$-convergence.

Proposition 4.7. If $\left\{F_{n}\right\}_{n \in \mathbb{N}}$ is a decreasing sequence converging to $F$ pointwise, then $\left\{F_{n}\right\}_{n \in \mathbb{N}}$ is $\Gamma$-convergent to $s c^{-} F$.

We conclude by giving a criterium for convergence of minima for $\Gamma$-convergent sequences (for a proof see [15, Theorem 7.8]).

Theorem 4.8. Suppose that the sequence $\left\{F_{n}\right\}_{n \in \mathbb{N}}$ is equi-coercive in $X$ and $\Gamma$-converges to a function $F$ in $X$. Then, $F$ is coercive and

$$
\min _{x \in X} F(x)=\lim _{n \rightarrow \infty} \inf _{x \in X} F_{n}(x) .
$$

\section{The large Deviation aCtion functional}

For any fixed $\varepsilon, \delta \in(0,1]$ and $\phi \in \mathrm{H}$, we consider the problem

$$
d u(t)+\mathrm{A} u(t)+\mathrm{B}(u(t), u(t))=\sqrt{\varepsilon} d w^{Q_{\delta}}(t), \quad u(0)=\phi,
$$

where

$$
w^{Q_{\delta}}(t)=\sum_{k=1}^{\infty} Q_{\delta} e_{k} \beta_{k}(t), \quad t \geq 0,
$$

$\left\{e_{k}\right\}_{k \in \mathbb{N}}$ is the basis which diagonalizes the operator $\mathrm{A},\left\{\beta_{k}\right\}_{k \in \mathbb{N}}$ is a sequence of independent Brownian motions all defined on the stochastic basis $(\Omega, \mathcal{F}, \mathbb{F}, \mathbb{P})$, where $\mathbb{F}=\left(\mathcal{F}_{t}\right)_{t \geq 0}$, and $Q_{\delta}$ is a bounded linear operator on $\mathrm{H}$, for any $\delta \in(0,1]$.

In what follows, we shall assume that the family $\left\{Q_{\delta}\right\}_{\delta \in(0,1]}$ satisfies the following conditions. 
Assumption 5.1. For every $\delta \in(0,1], Q_{\delta}$ is a positive linear operator on $\mathrm{H}$, the operator $A^{-1} Q_{\delta}^{2}$ is trace class, and there exists some $\beta>0$ such that $Q_{\delta}: \mathrm{H} \rightarrow D\left(\mathrm{~A}^{\frac{\beta}{2}}\right)$ is an isomorhism. Moreover,

$$
\lim _{\delta \rightarrow 0} Q_{\delta} y=y, \quad y \in \mathrm{H}, \quad \lim _{\delta \rightarrow 0} Q_{\delta}^{-1} y=y, \quad y \in D\left(\mathrm{~A}^{\frac{\beta}{2}}\right),
$$

the limites above being in $\mathrm{H}$, and for any $1 \geq \sigma \geq \delta \geq 0$

$$
\left|Q_{\sigma}^{-1} y\right|_{\mathrm{H}} \geq\left|Q_{\delta}^{-1} y\right|_{\mathrm{H}}, \quad y \in D\left(\mathrm{~A}^{\frac{\beta}{2}}\right) .
$$

Remark 2. The reproducing kernel Hilbert space of the Wiener process $w^{Q_{\delta}}$ is equal to $Q_{\delta}(\mathrm{H})$ and hence by Assumption 5.1 it coincides with the space $D\left(\mathrm{~A}^{\beta}\right)$ for some $\beta>0$. This implies that the results from [9] are applicable.

Remark 3. It is easy to see that for the Navier-Stokes equations in a $d$-dimensional domain, $d \geq 2$, any number $\beta>\frac{d}{2}-1$ and the operators

$$
Q_{\delta}:=\left(I+\delta \mathrm{A}^{\beta / 2}\right)^{-1}
$$

satisfy Assumption 5.1

Now, for any $-\infty \leq t_{0}<t_{1} \leq \infty, \delta \in[0,1]$ and $u \in C\left(\left[t_{0}, t_{1}\right] ; H\right)$, we define

$$
S_{t_{0}, t_{1}}^{\delta}(u):=\frac{1}{2} \int_{t_{0}}^{t_{1}}\left|Q_{\delta}^{-1}(\mathcal{H}(u)(t))\right|_{\mathrm{H}}^{2} d t,
$$

where $\mathcal{H}(u)$ is defined as in (3.23), with the usual convention that $S_{t_{0}, t_{1}}^{\delta}(u)=+\infty$, if $Q_{\delta}^{-1}(\mathcal{H}(u)(\cdot)) \notin L^{2}\left(t_{1}, t_{2} ; \mathrm{H}\right)$.

When $\delta=0$, the superscript 0 will be omitted. So we put $S_{t_{0}, t_{1}}=S_{t_{0}, t_{1}}^{0}$. Note that according to Lemma 3.9 a necessary condition for $S_{t_{0}, t_{1}}^{\delta}(u)$ to be finite is that $u\left(T_{1}\right) \in D\left(\mathrm{~A}^{\frac{\alpha+1}{2}}\right)$ and $u \in W^{1,2}\left(t_{2}, t_{1} ; D\left(\mathrm{~A}^{\frac{\alpha}{2}+1}\right), D\left(\mathrm{~A}^{\frac{\alpha}{2}}\right)\right)$, for any $t_{2} \in\left(t_{0}, t_{1}\right)$.

For any $T>0, p \geq 1, \varepsilon, \delta \in(0,1]$ and $\phi \in \mathrm{H}$, equation (5.1) admits a unique solution $u_{\phi}^{\varepsilon, \delta} \in L^{p}(\Omega ; C([0, T] ; H))$; for a proof see e.g. the fundamental work of Flandoli [21]. To be more precise let us now formulate a definition of a solution following [9].

Definition 5.2. If $u_{0} \in \mathrm{H}$, then an $\mathbb{F}$-adapted process $u(t), t \geq 0$ with trajectories in $C([0, \infty) ; \mathrm{H}) \cap L_{\text {loc }}^{4}\left([s, \infty) ; \mathbb{L}^{4}(D)\right)$ is a solution to problem (5.1) iff for any $v \in \mathrm{V}, t \geq 0$, $\mathbb{P}$-almost surely,

$$
\begin{aligned}
(u(t), v) & =\left(u_{0}, v\right)-\nu \int_{s}^{t}(u(r), \mathrm{A} v) d r-\int_{s}^{t} b(u(r), u(r), v) d r \\
& +\sqrt{\varepsilon}\left(v, w^{Q_{\delta}}(t)\right) .
\end{aligned}
$$

As shown in the next theorem, as an immediate consequence of the contraction principle, we have that the family $\left\{\mathcal{L}\left(u_{\phi}^{\varepsilon, \delta}\right)\right\}_{\varepsilon \in(0,1]}$ satisfies a large deviation principle in $C([0, T] ; \mathrm{H})$. 
Theorem 5.3. For any $x \in \mathrm{H}$ and $\delta \in(0,1]$, the family $\left\{\mathcal{L}\left(u_{\phi}^{\varepsilon, \delta}\right)\right\}_{\varepsilon \in(0,1]}$ satisfies a large deviation principle on $C([0, T] ; \mathrm{H})$, uniformly with respect to initial data $\phi$ in bounded sets of $\mathrm{H}$, with good action functional $S_{T}^{\delta}$.

Proof. For every $\varepsilon>0$ and $\delta \in(0,1]$, we denote by $z_{\varepsilon, \delta}(t)$ the Ornstein-Uhlenbeck process associated with $\mathrm{A}$ and $Q_{\delta}$, that is the solution of the linear problem

$$
d z(t)+\mathrm{A} z(t)=\sqrt{\varepsilon} d w^{Q_{\delta}}(t), \quad z(0)=0 .
$$

We have

$$
z_{\varepsilon, \delta}(t)=\sqrt{\varepsilon} \int_{0}^{t} e^{-(t-s) \mathrm{A}} d w^{Q_{\delta}}(s), \quad t \geq 0 .
$$

As well known (see e.g. [47, Theorem 3]), under our assumptions the family $\left\{\mathcal{L}\left(z_{\varepsilon, \delta}\right)\right\}_{\varepsilon \in(0,1]}$ satisfies a large deviation principle in $C\left([0, T] ; L^{4}(\mathcal{O})\right)$, with good action functional

$$
I_{0, T}^{\delta}(u)=\frac{1}{2} \int_{0}^{T}\left|Q_{\delta}^{-1}\left(u^{\prime}(t)+\mathrm{A} u(t)\right)\right|_{\mathrm{H}}^{2} d t .
$$

Moreover, if we define the mapping $\mathcal{F}: \mathrm{H} \times C\left([0, T] ; L^{4}(\mathcal{O})\right) \rightarrow C([0, T] ; \mathrm{H})$ which associates to every $\phi \in \mathrm{H}$ and $g \in C\left([0, T] ; L^{4}(\mathcal{O})\right)$ the solution $v \in C([0, T] ; \mathrm{H})$ of the problem

$$
v^{\prime}(t)+\mathrm{A} v(t)+B(v(t)+g(t), v(t)+g(t))=0, \quad v(0)=\phi,
$$

we have, $\mathbb{P}$-a.s.,

$$
u_{\phi}^{\varepsilon, \delta}=\mathcal{F}\left(\phi, z_{\varepsilon, \delta}\right) .
$$

Since, by [9, Theorem 4.6], the mapping $\mathcal{F}: \mathrm{H} \times C\left([0, T] ; L^{4}(\mathcal{O})\right) \rightarrow C([0, T] ; \mathrm{H})$ is continuous, by the contraction principle, the large deviation principle for $\left\{z_{\varepsilon, \delta}\right\}_{\varepsilon \in(0,1]}$ on $C\left([0, T] ; L^{4}(\mathcal{O})\right)$ with action functional $I_{0, T}^{\delta}$ may be transferred to a large deviation principle for $\left\{u_{\phi}^{\varepsilon, \delta}\right\}_{\varepsilon \in(0,1]}$ on $C([0, T] ; \mathrm{H})$, with action functional $S_{0, T}^{\delta}$.

Moreover, in [9, Theorem 4.6] it is shown that for any $R>0$ there exists $c_{R}>0$ such that for any $z_{1}, z_{2} \in B_{R}\left(C\left([0, T] ; L^{4}(\mathcal{O})\right)\right)$

$$
\sup _{\phi \in B_{0}(R)}\left|\mathcal{F}\left(\phi, z_{1}\right)-\mathcal{F}\left(\phi, z_{2}\right)\right|_{C([0, T] ; H)} \leq c_{R}\left|z_{1}-z_{2}\right|_{C\left([0, T] ; L^{4}(\mathcal{O})\right)} .
$$

This implies that the large deviation principle proved above is uniform with respect to the initial data $\phi$ in any bounded subset of $\mathrm{H}$.

In what follows, for any $T \in(0,+\infty]$ we set

$$
S_{T}^{\delta}:=S_{0, T}^{\delta}, \quad S_{-T}^{\delta}:=S_{-T, 0}^{\delta}
$$

and

In particular,

$$
S_{T}:=S_{0, T}, \quad S_{-T}:=S_{-T, 0}
$$

$$
S_{-\infty}^{\delta}(u):=\frac{1}{2} \int_{-\infty}^{0}\left|Q_{\delta}^{-1}(\mathcal{H}(u)(t))\right|_{\mathrm{H}}^{2} d t
$$


and

$$
S_{-\infty}(u):=\frac{1}{2} \int_{-\infty}^{0}|\mathcal{H}(u)(t)|_{\mathrm{H}}^{2} d t
$$

We conclude the present section with the description of some relevant properties of the functionals $S_{-\infty}$ and $S_{-\infty}^{\delta}$.

To this purpose, we need to introduce the following functional spaces

$$
\mathcal{X}=\left\{u \in C((-\infty, 0] ; \mathrm{H}): \lim _{t \rightarrow-\infty}|u(t)|_{\mathrm{H}}=0\right\}, \quad \mathcal{X}_{\phi}=\{u \in \mathcal{X}: u(0)=\phi\} .
$$

We endow the space $\mathcal{X}$ with the topology of uniform convergence on compact intervals, i.e. the topology induced by the metric $\rho$ defined by

$$
\rho(u, v):=\sum_{n=1}^{\infty} 2^{-n}\left(\sup _{s \in[-n, 0]}|u(s)-v(s)|_{\mathrm{H}} \wedge 1\right), \quad u, v \in \mathcal{X} .
$$

The set $\mathcal{X}_{\phi}$ is closed in $\mathcal{X}$ and we endow it with the trace topology induced by $\mathcal{X}$.

Let us note here, see for instance [46, Proposition I.3.1], that if $u \in W^{1,2}\left(t_{0}, \infty\right)$, then

$$
\lim _{t \rightarrow \infty}|u(t)|_{\mathrm{V}}=0 \text {. }
$$

Similarly, as shown in Proposition A.3, if $u \in W^{1,2}\left(-\infty, t_{1}\right)$, then

$$
\lim _{t \rightarrow-\infty}|u(t)|_{\mathrm{V}}=0 \text {. }
$$

Proposition 5.4. The functionals $S_{-\infty}$ and $S_{-\infty}^{\delta}, \delta \in(0,1]$, are lower-semicontinuous in $\mathcal{X}$.

Proof. In order to prove the lower semi-continuity of $S_{-\infty}$ and $S_{-\infty}^{\delta}$, it is sufficient to show that if a $\mathcal{X}$-valued sequence $\left\{u_{n}\right\}_{n=1}^{\infty}$ is convergent in $\mathcal{X}$ to a function $u \in \mathcal{X}$, then for any $\delta \in[0,1]$

$$
\liminf _{n \rightarrow \infty} S_{-\infty}^{\delta}\left(u_{n}\right) \geq S_{-\infty}^{\delta}(u) .
$$

First, we assume that $u \in \mathcal{X}$ is such that $S_{-\infty}^{\delta}(u)=\infty$. We want to show that

$$
\liminf _{n \rightarrow \infty} S_{-\infty}^{\delta}\left(u_{n}\right)=+\infty \text {. }
$$

Suppose by contradiction that $\lim \inf _{n} S_{-\infty}^{\delta}\left(u_{n}\right)<\infty$. Then, after extracting a subsequence, we can find $C>0$ such that

$$
\left|u_{n}^{\prime}+A u_{n}+B\left(u_{n}, u_{n}\right)\right|_{L^{2}\left(-\infty, 0 ; D\left(\mathrm{~A}^{\frac{\beta}{2}}\right)\right)} \leq C, \quad n \in \mathbb{N} .
$$

By Proposition A.2 (Proposition A.1, if $\delta=0$ ), we have that the sequence $\left\{u_{n}\right\}$ is bounded in $W^{1,2}\left(-\infty, 0 ; D\left(\mathrm{~A}^{1+\frac{\beta}{2}}\right), D\left(\mathrm{~A}^{\frac{\beta}{2}}\right)\right)$ and hence we can find $\tilde{u} \in W^{1,2}\left(-\infty, 0 ; D\left(\mathrm{~A}^{1+\frac{\beta}{2}}\right), D\left(\mathrm{~A}^{\frac{\beta}{2}}\right)\right)$ such that, after another extraction of a subsequence,

$$
u_{n} \rightarrow \tilde{u} \text {, as, } n \rightarrow \infty \text { weakly in } W^{1,2}\left(-\infty, 0 ; D\left(\mathrm{~A}^{1+\frac{\beta}{2}}\right), D\left(\mathrm{~A}^{\frac{\beta}{2}}\right)\right) .
$$

By the uniqueness of the limit we infer that $u=\tilde{u}$, so that $u \in W^{1,2}\left(-\infty, 0 ; D\left(\mathrm{~A}^{1+\frac{\beta}{2}}\right), D\left(\mathrm{~A}^{\frac{\beta}{2}}\right)\right)$ and $S_{-\infty}^{\delta}(u)<\infty$, which contradicts our assumption. 
Thus, assume that $S_{-\infty}^{\delta}(u)<\infty$. In view of the last part of Lemma 3.9 (Lemma 3.8 if $\delta=0$ ) we have that $u(0) \in D\left(\mathrm{~A}^{\frac{1+\beta}{2}}\right)$ and $u \in W^{1,2}\left(-\infty, 0 ; D\left(\mathrm{~A}^{1+\frac{\beta}{2}}\right), D\left(\mathrm{~A}^{\frac{\beta}{2}}\right)\right)$.

Now, assume that (5.8) is not true. Then there exists $\varepsilon>0$ such that, after the extraction of a subsequence,

$$
S_{-\infty}^{\delta}\left(u_{n}\right)<S_{-\infty}^{\delta}(u)-\varepsilon, \quad n \in \mathbb{N} .
$$

Hence, if we set $f_{n}=u_{n}^{\prime}+A u_{n}+B\left(u_{n}, u_{n}\right)$, we have that the sequence $\left\{f_{n}\right\}$ is bounded in $L^{2}\left(-\infty, 0 ; D\left(\mathrm{~A}^{\frac{\beta}{2}}\right)\right)$ and then, by Proposition A.2 (Proposition A.1 if $\delta=0$ ), we have that $\left\{u_{n}\right\}$ is bounded in $W^{1,2}\left(-\infty, 0 ; D\left(\mathrm{~A}^{1+\frac{\beta}{2}}\right), D\left(\mathrm{~A}^{\frac{\beta}{2}}\right)\right)$. This implies that we can find $\tilde{u} \in$ $W^{1,2}\left(-\infty, 0 ; D\left(\mathrm{~A}^{1+\frac{\beta}{2}}\right), D\left(\mathrm{~A}^{\frac{\beta}{2}}\right)\right)$ such that, after a further extraction of a subsequence,

$$
u_{n}=\tilde{u}, \quad \text { as } n \rightarrow \infty \text {, weakly in } W^{1,2}\left(-\infty, 0 ; D\left(\mathrm{~A}^{1+\frac{\beta}{2}}\right), D\left(\mathrm{~A}^{\frac{\beta}{2}}\right)\right),
$$

and, by uniqueness of the limit, we infer that $u=\tilde{u}$. Moreover, as the sequence $\left\{f_{n}\right\}$ is bounded in $L^{2}\left(-\infty, 0 ; D\left(\mathrm{~A}^{\frac{\beta}{2}}\right)\right)$, after another extraction of a subsequence, we can find $\tilde{f} \in$ $L^{2}\left(-\infty, 0 ; D\left(\mathrm{~A}^{\frac{\beta}{2}}\right)\right)$ such that $f_{n}$ converges weakly to $\tilde{f}$ in $L^{2}\left(-\infty, 0 ; D\left(\mathrm{~A}^{\frac{\beta}{2}}\right)\right)$. By employing nowadays standard compactness argument, see for instance [9, section 5] we can show that $\tilde{f}=f=u^{\prime}+\mathrm{A} u+\mathrm{B}(u, u)$. Thus, since the mapping

$$
f \in L^{2}\left(-\infty, 0 ; D\left(\mathrm{~A}^{\frac{\beta}{2}}\right)\right) \mapsto \int_{-\infty}^{0}\left|Q_{\delta}^{-1} f(t)\right|_{H}^{2} d t \in \mathbb{R}
$$

is convex and lower semi-continuous, it is also weakly lower semi-continuous, so that

$$
\liminf _{n \rightarrow \infty} S_{-\infty}^{\delta}\left(u_{n}\right)=\frac{1}{2} \liminf _{n \rightarrow \infty}\left|Q_{\delta}^{-1} f_{n}\right|_{L^{2}\left(-\infty, 0 ; D\left(\mathrm{~A}^{\frac{\beta}{2}}\right)\right)}^{2} \geq \frac{1}{2}\left|Q_{\delta}^{-1} f\right|_{L^{2}\left(-\infty, 0 ; D\left(\mathrm{~A}^{\frac{\beta}{2}}\right)\right)}^{2}=S_{-\infty}^{\delta}(u) .
$$

Proposition 5.5. The operators $S_{-\infty}$ and $S_{-\infty}^{\delta}$ have compact level sets in $\mathcal{X}$. Moreover, the family $\left\{S_{-\infty}^{\delta}\right\}_{\delta \in(0,1]}$ is equi-coercive.

Proof. First, notice that we have only to prove the compactness of the level sets of $S_{-\infty}$. Actually, due to Assumption 5.1.

$$
S_{-\infty}^{\delta} \geq S_{-\infty}, \quad \delta \in(0,1]
$$

and then, as $S_{-\infty}^{\delta}$ is lower-semicontinuous, the compactness of the level sets of $S_{-\infty}$ implies the compactness of the level sets of $S_{-\infty}^{\delta}$. Moreover, in view of Proposition 4.3, (5.9) and the compactness of the level sets of $S_{-\infty}$ imply the equi-coercivity of the family $\left\{S_{-\infty}^{\delta}\right\}_{\delta \in(0,1]}$. Hence, we have only to prove that every sequence $\left\{u_{n}\right\}$ in $\mathcal{X}$ such that $S_{-\infty}\left(u_{n}\right) \leq r$, for any $n \in \mathbb{N}$, has a subsequence convergent in $\mathcal{X}$ to some $u \in \mathcal{X}$ such that $S_{-\infty}(u) \leq r$.

According to the last part of Proposition A.1 there exists $M>0$ such that

$$
\left|u_{n}\right|_{W^{1,2}(-\infty, 0)} \leq M, \quad n \in \mathbb{N} .
$$


Hence by the Banach-Alaoglu Theorem, we can find $u \in W^{1,2}(-\infty, 0)$ and a subsequence of the original sequence that is weakly convergent to $u$ in $W^{1,2}(-\infty, 0)$. Note that $u$ being an element of $W^{1,2}(-\infty, 0)$ it must satisfy

$$
\lim _{t \rightarrow-\infty}|u(t)|_{\mathrm{H}}=0 .
$$

Since the embedding $D(\mathrm{~A}) \hookrightarrow \mathrm{H}$ is compact, by the last part of [8, Corollary 2.8] with $\alpha=1$ and $q=2$, we infer that that for each $T>0$ the embedding $W^{1,2}(-T, 0) \hookrightarrow C([-T, 0], \mathrm{H})$ is compact. Hence, for each $T>0$ we can extract a subsequence strongly convergent in $C([-T, 0], \mathrm{H})$. By the uniqueness of the limit we infer that the later limit is equal to the restriction of $u$ to the interval $[-T, 0]$. In particular $u \in \mathcal{X}$. Moreover, by employing the Helly's diagonal procedure, we can find a subsequence of $\left\{u_{n}\right\}$ which is convergent in $\mathcal{X}$ to $u$ and, as $S_{-\infty}$ is lower semicontinuous, we have that $S_{-\infty}(u) \leq r$. This completes the proof of the compactness of the level sets of $S_{-\infty}$.

\section{THE QUASI-POTENTIAL}

We define, for $\phi \in \mathrm{H}$, the following of $[0, \infty]$-valued functions

$$
U(\phi):=\inf \left\{S_{-T}(u): T>0, u \in C([-T, 0] ; \mathrm{H}), \text { with } u(-T)=0, u(0)=\phi\right\},
$$

and for any $\delta \in(0,1]$

$$
U_{\delta}(\phi):=\inf \left\{S_{-T}^{\delta}(u): T>0, u \in C([-T, 0] ; \mathrm{H}), \text { with } u(-T)=0, u(0)=\phi\right\} .
$$

Note that with our notation $U=U_{0}$.

As a consequence of Lemma 3.8 and Lemma 3.9, we have the following result.

Proposition 6.1. We have

$$
U(\phi)<\infty \Longleftrightarrow \phi \in \mathrm{V} .
$$

Moreover, if Assumption 5.1 is satisfied for some $\beta \in\left(0, \frac{1}{2}\right)$, then we have

$$
U_{\delta}(\phi)<\infty \Longleftrightarrow \phi \in D\left(\mathrm{~A}^{\frac{\beta+1}{2}}\right) .
$$

Proof. We prove (6.4), as (6.3) turns out to be a special case, corresponding to the case $\beta=0$. Assume that $U_{\delta}(\phi)<\infty$. Then, according to (6.2) we can find $T>0$ and $u \in C([-T, 0] ; \mathrm{H})$ such that $u(-T)=0, u(0)=\phi$ and

$$
u^{\prime}+\mathrm{A} u+\mathrm{B}(u, u) \in L^{2}\left(-T, 0 ; D\left(\mathrm{~A}^{\frac{\beta}{2}}\right)\right) .
$$

Hence, by Lemma 3.9 we infer that $\phi \in D\left(\mathrm{~A}^{\frac{1+\beta}{2}}\right)$.

Conversely, let us assume that $\phi \in D\left(\mathrm{~A}^{\frac{1+\beta}{2}}\right)$. Since for any $T>0$ the map

$$
W\left(-T, 0 ; D\left(\mathrm{~A}^{1+\frac{\beta}{2}}\right), D\left(\mathrm{~A}^{\frac{\beta}{2}}\right)\right) \ni v \mapsto v(0) \in D\left(\mathrm{~A}^{\frac{1+\beta}{2}}\right)
$$

is surjective, see [30, Theorem 3.2, p.21 and Remark 3.3, p.22] for a proof, we can find $u_{1} \in W\left(-T, 0 ; D\left(\mathrm{~A}^{1+\frac{\beta}{2}}\right), D\left(\mathrm{~A}^{\frac{\beta}{2}}\right)\right)$ such that $u_{1}(0)=\phi$. By Proposition 2.5, we infer that 
$u_{1}^{\prime}+A u_{1}+B\left(u_{1}, u_{1}\right) \in L^{2}\left(-T, 0 ; D\left(\mathrm{~A}^{\frac{\beta}{2}}\right)\right)$. Moreover, there exists $t_{0} \in(-T, 0)$ such that $u_{1}\left(t_{0}\right) \in D\left(\mathrm{~A}^{1+\frac{\beta}{2}}\right)$. This means that if we define

$$
u_{2}(t):=\frac{t+T}{t_{0}+T} u_{1}\left(t_{0}\right), \quad t \in\left[-T, t_{0}\right]
$$

we have that $u_{2}(-T)=0, u_{2} \in W\left(-T, t_{0} ; D\left(\mathrm{~A}^{1+\frac{\beta}{2}}\right), D\left(\mathrm{~A}^{\frac{\beta}{2}}\right)\right)$ and $u_{2}^{\prime}+A u_{2}+B\left(u_{2}, u_{2}\right) \in$ $L^{2}\left(-T, t_{0} ; D\left(\mathrm{~A}^{\frac{\beta}{2}}\right)\right)$. Finally, if we define

$$
u(t):= \begin{cases}u_{2}(t), & t \in\left[-T, t_{0}\right] \\ u_{1}(t), & t \in\left[t_{0}, 0\right],\end{cases}
$$

we can conclude that $S_{-T}^{\delta}(u)<\infty$.

Now we can prove the following crucial characterization of the functionals $U_{\delta}$ and $U$.

Theorem 6.2. For any $\phi \in \mathrm{V}$ we have

$$
U(\phi):=\min \left\{S_{-\infty}(u): u \in \mathcal{X}_{\phi}\right\} .
$$

Analogously, if Assumption [5.1 is satisfied for some $\beta \in\left(0, \frac{1}{2}\right)$, then for any $\delta \in(0,1]$ and $\phi \in D\left(\mathrm{~A}^{\frac{1+\beta}{2}}\right)$ we have

$$
U_{\delta}(\phi):=\min \left\{S_{-\infty}^{\delta}(u): u \in \mathcal{X}_{\phi}\right\} .
$$

Proof. We prove (6.6), as (6.5) is a special case, corresponding to $\beta=0$ in Assumption 5.1. Let us fix $T>0$ and $u \in C([-T, 0] ; \mathrm{H})$ such that $u(-T)=0, u(0)=\phi$ and $S_{-T}^{\delta}(u)<\infty$ and let us define

$$
\bar{u}(t):= \begin{cases}u(t), & \text { if } t \in[-T, 0], \\ 0, & \text { if } t \in(-\infty,-T] .\end{cases}
$$

Obviously, $\bar{u} \in \mathcal{X}_{\phi}$. We will prove that

$$
S_{-\infty}^{\delta}(\bar{u})=S_{-T}^{\delta}(u)
$$

Since $S_{-T}^{\delta}(u)<\infty$, the function $u$ satisfies the assumptions of Lemma 3.9. Therefore, $\phi \in$ $D\left(\mathrm{~A}^{\frac{1+\beta}{2}}\right)$ and $u$ belongs to $W^{1,2}\left(-T, 0 ; D\left(\mathrm{~A}^{1+\frac{\beta}{2}}\right), D\left(\mathrm{~A}^{\frac{\beta}{2}}\right)\right)$. Since obviously the zero function is an elements of the space $W^{1,2}\left(-\infty,-T ; D\left(\mathrm{~A}^{1+\frac{\beta}{2}}\right), D\left(\mathrm{~A}^{\frac{\beta}{2}}\right)\right)$, we infer, see for instance [5], that

$$
\bar{u} \in W^{1,2}\left(-\infty, 0 ; D\left(\mathrm{~A}^{1+\frac{\beta}{2}}\right), D\left(\mathrm{~A}^{\frac{\beta}{2}}\right)\right),
$$

and (6.8) holds. In particular, this implies that

$$
\inf \left\{S_{-\infty}^{\delta}(u): u \in \mathcal{X}_{\phi}\right\} \leq S_{-T}^{\delta}(u) .
$$

Taking now the infimum over all $u$ as above, in view of the definition of $U_{\delta}(\phi)$ we infer that

$$
\inf \left\{S_{-\infty}^{\delta}(u): u \in \mathcal{X}_{\phi}\right\} \leq U_{\delta}(\phi) .
$$


It remains to prove the converse inequality. To this purpose, we will need the following two results, whose proofs are postponed to the end of this section.

Lemma 6.3. For every $\delta \in[0,1], T>0$ and $\varepsilon>0$, there exists $\eta>0$ such that for any $y \in D\left(\mathrm{~A}^{\frac{1+\beta}{2}}\right)$ such that $|y|_{D\left(\mathrm{~A}^{\frac{1+\beta}{2}}\right)}<\eta$, we can find

$$
v \in W\left(0, T ; D\left(\mathrm{~A}^{1+\frac{\beta}{2}}\right), D\left(\mathrm{~A}^{\frac{\beta}{2}}\right)\right)
$$

with

$$
S_{T}^{\delta}(v)<\varepsilon, \quad v(0)=0, \quad v(T)=y .
$$

Recall that in the case $\delta=0$, we have $S_{T}^{\delta}=S_{T}$ and we take $\beta=0$.

Lemma 6.4. Assume that $u \in \mathcal{X}$. Then for each $\delta \in[0,1]$ and $\varepsilon>0$ we can find $T_{\varepsilon}>0$ and $v_{\varepsilon} \in C\left(\left[-T_{\varepsilon}, 0\right] ; \mathrm{H}\right)$ such that $v_{\varepsilon}\left(-T_{\varepsilon}\right)=0, v_{\varepsilon}(0)=u(0)$ and

$$
S_{-T_{\varepsilon}}^{\delta}\left(v_{\varepsilon}\right) \leq S_{-\infty}^{\delta}(u)+\varepsilon
$$

Thus, let us prove

$$
U_{\delta}(\phi) \leq \inf \left\{S_{-\infty}^{\delta}(u): u \in \mathcal{X}_{\phi}\right\}
$$

Obviously, we may assume that the right hand side above is finite and so we can find $u \in \mathcal{X}_{\phi}$ such that $S_{-\infty}^{\delta}(u)<\infty$. In view of Lemma 6.4, for any $\varepsilon>0$,

$$
\inf \left\{S_{-T}^{\delta}(v): T>0, v \in C([-T, 0], \mathrm{H}), v(-T)=0, v(0)=\phi\right\} \leq S_{-\infty}^{\delta}(u)+\varepsilon .
$$

This implies that $U_{\delta}(\phi) \leq S_{-\infty}^{\delta}(u)+\varepsilon$. Thus, by taking the infimum over $\varepsilon>0$ and then over all admissible $u$ we get (6.9). Finally, we remark that the infima are in fact minima, as the level sets of $S_{-\infty}$ and $S_{-\infty}^{\delta}$ are compact (see Proposition 5.5).

This completes the proof of ([6.6), provided we can prove Lemmas 6.3 and 6.4 .

Proof of Lemma 6.3. Let us fix $T>0$ and consider the mapping

$$
\mathcal{H}_{T}: W^{1,2}\left(0, T ; D\left(\mathrm{~A}^{1+\frac{\beta}{2}}\right), D\left(\mathrm{~A}^{\frac{\beta}{2}}\right)\right) \ni v \mapsto v^{\prime}+\mathrm{A} v+\mathrm{B}(v, v) \in L^{2}\left(0, T ; D\left(\mathrm{~A}^{\frac{\beta}{2}}\right)\right) .
$$

Due to Lemma 2.5, the mapping $\mathcal{H}_{T}$ is well defined and continuous. Moreover

$$
S_{T}^{\delta}(v) \leq c\left|\mathcal{H}_{T}(v)\right|_{L^{2}\left(0, T ; D\left(\mathrm{~A}^{\frac{\beta}{2}}\right)\right)}, \quad v \in W^{1,2}\left(0, T ; D\left(\mathrm{~A}^{1+\frac{\beta}{2}}\right), D\left(\mathrm{~A}^{\frac{\beta}{2}}\right)\right) .
$$

Now, by proceeding as in [30, Remark 3.3, p. 22] we can show that there exists a continuous linear map

$$
R: D\left(\mathrm{~A}^{\frac{1+\beta}{2}}\right) \rightarrow W^{1,2}\left(0, T ; D\left(\mathrm{~A}^{1+\frac{\beta}{2}}\right), D\left(\mathrm{~A}^{\frac{\beta}{2}}\right)\right)
$$

such that $[R y](T)=y$ for every $y \in D\left(\mathrm{~A}^{\frac{1+\beta}{2}}\right)$. By using the same augments used in the proof of Proposition 6.1, we can construct Ry such that $R y(0)=0$. Thus the map

$$
\mathcal{H}_{T} \circ R: D\left(\mathrm{~A}^{\frac{1+\beta}{2}}\right) \rightarrow L^{2}\left(0, T ; D\left(\mathrm{~A}^{\frac{\beta}{2}}\right)\right)
$$

is continuous and then for every $\varepsilon>0$ we can find $\eta>0$ such that

$$
|y|_{D\left(\mathrm{~A}^{\frac{1+\beta}{2}}\right)}<\eta \Longrightarrow\left|\mathcal{H}_{T}(R y)\right|_{L^{2}\left(0, T ; D\left(\mathrm{~A}^{\frac{\beta}{2}}\right)\right.}<\frac{\varepsilon}{c}
$$


Since $v=R y$ satisfies $v \in W^{1,2}\left(0, T ; D\left(\mathrm{~A}^{1+\frac{\beta}{2}}\right), D\left(\mathrm{~A}^{\frac{\beta}{2}}\right)\right), v(0)=0$ and $v(T)=y$, due to (6.10) the proof is complete.

Proof of Lemma 6.4. We give the proof here for $\delta>0$, as $\delta=0$ is a special case. Let us assume that $u \in \mathcal{X}_{\phi}$ for some $\phi \in H$, and fix $\varepsilon>0$. We can assume that $S_{-\infty}^{\delta}(u)<\infty$. Then by (5.5) we can find $T_{\varepsilon}>0$ such that

$$
S_{-\infty,-T_{\varepsilon}}^{\delta}(u)<\frac{\varepsilon}{3}
$$

Moreover, the function $u$ satisfies the assumptions of Lemma 3.9. Therefore, $\phi \in D\left(\mathrm{~A}^{\frac{1+\beta}{2}}\right)$ and $u$ belongs to $W_{\text {loc }}^{1,2}\left(-\infty, 0 ; D\left(\mathrm{~A}^{1+\frac{\beta}{2}}\right), D\left(\mathrm{~A}^{\frac{\beta}{2}}\right)\right)$. As a consequence of Proposition A.2, this implies

$$
\lim _{t \rightarrow-\infty}|u(t)|_{D\left(\mathrm{~A}^{\frac{1+\beta}{2}}\right)}=0 .
$$

Then, $T_{\varepsilon}$ can be chosen in such a way that

$$
\left|u\left(-T_{\varepsilon}\right)\right|_{D\left(\mathrm{~A} \frac{1+\beta}{2}\right)}<\eta
$$

where we choose $\eta>0$ as in Lemma 6.3, corresponding to $T=1$ and $\frac{\varepsilon}{3}$. Then by Lemma 6.3, we can find $w \in W^{1,2}\left(-T_{\varepsilon}-1,-T_{\varepsilon} ; D\left(\mathrm{~A}^{1+\frac{\beta}{2}}\right), D\left(\mathrm{~A}^{\frac{\beta}{2}}\right)\right)$ such that

$$
S_{-T_{\varepsilon}-1,-T_{\varepsilon}}^{\delta}(w)<\frac{\varepsilon}{3}, \quad w\left(-T_{\varepsilon}-1\right)=0, \quad w\left(-T_{\varepsilon}\right)=u\left(-T_{\varepsilon}\right) .
$$

Next, we define

$$
\bar{u}(t):= \begin{cases}u(t), & \text { if } t \in\left[-T_{\varepsilon}, 0\right], \\ w(t), & \text { if } t \in\left[-T_{\varepsilon}-1,-T_{\varepsilon}\right] .\end{cases}
$$

Obviously, $\bar{u}(0)=\phi$ and $\bar{u} \in C\left(\left[-T_{\varepsilon}-1,0\right] ; \mathrm{H}\right)$ and, arguing as before (and hence using for instance [5]), we infer that $\bar{u} \in W^{1,2}\left(-T_{\varepsilon}-1,0 ; D\left(\mathrm{~A}^{1+\frac{\beta}{2}}\right), D\left(\mathrm{~A}^{\frac{\beta}{2}}\right)\right)$. Moreover,

$$
\begin{aligned}
S_{-T}^{\delta}(\bar{u}) & =S_{-T_{\varepsilon}-1,-T_{\varepsilon}}^{\delta}(w)+S_{-T_{\varepsilon}}^{\delta}(u) \\
& <\frac{\varepsilon}{3}+\left[S_{-\infty}^{\delta}(u)-S_{-\infty,-T_{\varepsilon}}^{\delta}(u)\right]<\frac{\varepsilon}{3}+S_{-\infty}^{\delta}(u) .
\end{aligned}
$$

This concludes the proof of Lemma 6.4.

Next, we prove that both $U$ and $U^{\delta}$ have compact level sets.

Proposition 6.5. For any $r>0$ and $\delta \in(0,1]$, the sets

$$
K_{r}=\{\phi \in \mathrm{H}: U(\phi) \leq r\}, \quad K_{r}^{\delta}=\left\{\phi \in \mathrm{H}: U_{\delta}(\phi) \leq r\right\}
$$

are compact in $\mathrm{H}$. In particular, both $U$ and $U^{\delta}$ are lower semi-continuous in $\mathrm{H}$. 
Proof. Let $\left\{\phi_{n}\right\}$ be a sequence in $K_{r}$. In view of identity (6.5), for any $n \in \mathbb{N}$ there exists $u_{n} \in \mathcal{X}_{\phi_{n}}$ such that

$$
S_{-\infty}\left(u_{n}\right)=U\left(\phi_{n}\right) \leq r
$$

In particular,

$$
\left\{u_{n}\right\} \subset\left\{S_{-\infty} \leq r\right\},
$$

so that, thanks to the compactness of the level sets of $S_{-\infty}$ proved in Proposition 5.5, we can find a subsequence $\left\{u_{n_{k}}\right\} \subset\left\{u_{n}\right\}$ and $\bar{u} \in C((-\infty, 0] ; \mathrm{H})$ such that

$$
\lim _{k \rightarrow \infty} u_{n_{k}}=\bar{u}, \quad \text { in } C((-\infty, 0] ; \mathrm{H}) \text {. }
$$

This implies that

$$
\lim _{k \rightarrow \infty} u_{n_{k}}(0)=\bar{u}(0)
$$

and, due to the lower semi-continuity of $S_{-\infty}$ proved in Proposition 5.4,

$$
S_{-\infty}(\bar{u}) \leq \liminf _{k \rightarrow \infty} S_{-\infty}\left(u_{n_{k}}\right) \leq r .
$$

On the other hand, by the definition of $U, U(\bar{u}(0)) \leq S_{-\infty}(\bar{u})$. Hence we can conclude that $\bar{u}(0) \in K_{r}$, and the compactness of $K_{r}$ follows.

The compactness of the level sets of $U_{\delta}$ can be proved analogously.

We conclude this section by studying the continuity of $U$ in $\mathrm{V}$ and of $U_{\delta}$ in $D\left(\mathrm{~A}^{\frac{1+\beta}{2}}\right)$.

Proposition 6.6. The maps $U: \mathrm{V} \rightarrow \mathbb{R}$ and $U_{\delta}: D\left(\mathrm{~A}^{\frac{1+\beta}{2}}\right) \rightarrow \mathbb{R}$ are continuous.

Proof. In the previous proposition we have seen that $U$ is lower semi-continuous in $\mathrm{H}$. In particular, it is lower semi-continuous in $\mathrm{V}$. Thus, is we prove that $U$ is also upper semicontinuous in $\mathrm{V}$, we can conclude that it is continuous on $\mathrm{V}$.

Let $\left\{\phi_{n}\right\}_{n \in \mathbb{N}}$ be a sequence in $\mathrm{V}$ converging to some $\phi$ in $\mathrm{V}$. As $\phi \in \mathrm{V}$, according to Proposition 6.1 and Theorem 6.2, there exists $u \in \mathcal{X}_{\phi} \cap W^{1,2}(-\infty, 0)$ such that $U(\phi)=$ $S_{-\infty}(u)$. Now, we define

$$
u_{n}(t)=u(t)+e^{t \mathrm{~A}}\left(\phi_{n}-\phi\right), \quad t \leq 0 .
$$

Clearly $u_{n}(0)=\phi_{n}$. Then, as $\phi_{n}-\phi \in \mathrm{V}$, we have that $u_{n} \in \mathcal{X}_{\phi_{n}} \cap W^{1,2}(-\infty, 0)$. Moreover, as $\phi_{n}$ converges to $\phi$ in $\mathrm{V}$ and $\mathrm{V}=(\mathrm{H}, D(\mathrm{~A}))_{\frac{1}{2}, 2}$, we infer that $u_{n}$ converges to $u$ in $W^{1,2}(-\infty, 0)$, so that

$$
\lim _{n \rightarrow \infty} S_{-\infty}\left(u_{n}\right)=S_{-\infty}(u)
$$

This allows to conclude that

$$
U(\phi)=S_{-\infty}(u)=\lim _{n \rightarrow \infty} S_{-\infty}\left(u_{n}\right) \geq \limsup _{n \rightarrow \infty} U\left(\phi_{n}\right),
$$

so that upper semi-continuity follows.

The proof of the continuity of the map $U_{\delta}: D\left(\mathrm{~A}^{\frac{1+\beta}{2}}\right) \rightarrow \mathbb{R}$ is analogous. 


\section{Stochastic Navier Stokes equations with Periodic boundary Conditions}

All what we have discussed throughout the paper until now applies to the case when the Dirichlet boundary conditions are replaced by the periodic boundary conditions. In the latter case, it is customary to study our problem in the 2-dimensional torus $\mathbb{T}^{2}$ (of fixed dimensions $L \times L)$, instead of a regular bounded domain $\mathcal{O}$. All the mathematical background can be found in the small book [40] by Temam. In particular, the space $\mathrm{H}$ is equal to

$$
H_{\infty}=\left\{u \in L_{0}^{2}\left(\mathbb{T}^{2}, \mathbb{R}^{2}\right): \operatorname{div}(u)=0 \text { and } \gamma_{\nu}(u)_{\mid \Gamma_{j+2}}=-\gamma_{\nu}(u)_{\mid \Gamma_{j}}, j=1,2\right\},
$$

where $L_{0}^{2}\left(\mathbb{T}^{2}, \mathbb{R}^{2}\right)$ is the Hilbert space consisting of those $u \in L^{2}\left(\mathbb{T}^{2}, \mathbb{R}^{2}\right)$ which satisfy $\int_{\mathbb{T}^{2}} u(x) d x=0$ and $\Gamma_{j}, j=1, \cdots, 4$ are the four (not disjoint) parts of the boundary of $\partial\left(\mathbb{T}^{2}\right)$ defined by

$$
\Gamma_{j}=\left\{x=\left(x_{1}, x_{2}\right) \in[0, L]^{2}: x_{j}=0\right\}, \Gamma_{j+2}=\left\{x=\left(x_{1}, x_{2}\right) \in[0, L]^{2}: x_{j}=L\right\}, \quad j=1,2 .
$$

The Stokes operator A can be defined in a natural way and it satisfies all the properties know in the bounded domain case, inclusive the positivity property (2.11) (with $\lambda_{1}=\frac{4 \pi^{2}}{L^{2}}$ ) and the following property involving the nonlinear term $B$

$$
\langle\mathrm{A} u, B(u, u)\rangle_{\mathrm{H}}=0, \quad u \in D(\mathrm{~A}),
$$

see [40, Lemma 3.1] for a proof. The Leray-Helmholtz projection operator $P$ has the following explicit formula using the Fourier series, see [40, (2.13)]

$$
[P(f)]_{k}=\frac{L^{2}}{4 \pi^{2}}\left(f_{k}-\frac{\left(k \cdot f_{k}\right) f_{k}}{|k|^{2}}\right), \quad k \in \mathbb{Z}^{2} \backslash\{0\}, f=\sum_{n \in \mathbb{Z}^{2} \backslash\{0\}} f_{n} e^{\frac{2 \pi i n \cdot x}{L}} \in L_{0}^{2}\left(\mathbb{T}^{2}, \mathbb{R}^{2}\right) .
$$

It follows from the above that $P$ is a bounded linear map from $D\left(\mathrm{~A}^{\alpha}\right)$ to itself for every $\alpha \geq 0$, compare with Proposition 2.1 in the bounded domain case.

In the next Theorem we will show that, in this case, an explicit representation of $U(x)$ can be given, for any $x \in \mathrm{V}$.

Theorem 7.1. Assume that periodic boundary conditions hold. Then

$$
U(\phi)= \begin{cases}|\phi|_{\mathrm{V}}^{2}, & \phi \in \mathrm{V} \\ +\infty, & \phi \in \mathrm{H} \backslash \mathrm{V}\end{cases}
$$

Proof. By Theorem 6.2, we have that

$$
U(\phi)=\min \left\{S_{-\infty}(u): u \in \mathcal{X}_{\phi}\right\}, \quad \phi \in \mathrm{V},
$$

and by Proposition 6.1 we have that $U(\phi)<\infty$ if and only if $\phi \in \mathrm{V}$. Now, let us fix $\phi \in \mathrm{V}$ and $u \in \mathcal{X}_{\phi}$ such that $S_{-\infty}(u)<\infty$. In view of Proposition A.1, we have that

$$
u \in C((-\infty, 0] ; \mathrm{V}) \cap L^{2}(-\infty, 0 ; D(\mathrm{~A})), \quad u^{\prime} \in L^{2}(-\infty, 0 ; \mathrm{H})
$$

and

$$
\lim _{t \rightarrow-\infty}|u(t)|_{\mathrm{V}}=0
$$


We have

$$
\begin{aligned}
& \left|u^{\prime}(t)+\mathrm{A} u(t)+\mathrm{B}(u(t), u(t))\right|_{\mathrm{H}}^{2}=\left|u^{\prime}(t)-\mathrm{A} u(t)+\mathrm{B}(u(t), u(t))\right|_{\mathrm{H}}^{2} \\
& +4|\mathrm{~A} u(t)|_{\mathrm{H}}^{2}+4\left\langle u^{\prime}(t)-\mathrm{A} u(t)+\mathrm{B}(u(t), u(t)), \mathrm{A} u(t)\right\rangle_{\mathrm{H}} .
\end{aligned}
$$

Then, thanks to (7.1) we get

$$
\left|u^{\prime}(t)+\mathrm{A} u(t)+\mathrm{B}(u(t), u(t))\right|_{\mathrm{H}}^{2}=\left|u^{\prime}(t)-\mathrm{A} u(t)+\mathrm{B}(u(t), u(t))\right|_{\mathrm{H}}^{2}+4\left\langle u^{\prime}(t), \mathrm{A} u(t)\right\rangle_{\mathrm{H}} .
$$

According to (7.2), this means that

$$
\begin{aligned}
& S_{-\infty}(u)=\frac{1}{2} \int_{-\infty}^{0}\left|u^{\prime}(t)-\mathrm{A} u(t)+\mathrm{B}(u(t), u(t))\right|_{\mathrm{H}}^{2} d t+\int_{-\infty}^{0} \frac{d}{d t}|u(t)|_{\mathrm{V}}^{2} d t \\
& =\frac{1}{2} \int_{-\infty}^{0}\left|u^{\prime}(t)-\mathrm{A} u(t)+\mathrm{B}(u(t), u(t))\right|_{\mathrm{H}}^{2} d t+|u(0)|_{\mathrm{V}}^{2} .
\end{aligned}
$$

In particular,

$$
U(\phi) \geq|\phi|_{\mathrm{V}}^{2}
$$

On the other hand, if we show that for any $\phi \in \mathrm{V}$ there exists $\bar{u} \in W^{1,2}(-\infty, 0) \cap \mathcal{X}_{\phi}$ such that $\bar{u}^{\prime}(t)-\mathrm{A} \bar{u}(t)+B(\bar{u}(t), \bar{u}(t))=0$, for $t \in(-\infty, 0)$, we conclude that $U(\phi)=|\phi|_{\mathrm{V}}^{2}$. As we have seen in Section 2, if $\phi \in \mathrm{V}$ then the problem

$$
\left\{\begin{array}{l}
v^{\prime}(t)+\mathrm{A} v(t)-\mathrm{B}(v(t), v(t))=0, \quad t>0 \\
v(0)=\phi
\end{array}\right.
$$

admits a unique solution $v \in L^{2}(0,+\infty ; D(\mathrm{~A})) \cap C([0,+\infty) ; \mathrm{V})$, with $v^{\prime} \in L^{2}(0,+\infty ; \mathrm{H})$, with

$$
\lim _{t \rightarrow \infty}|v(t)|_{\mathrm{V}}^{2}=0
$$

This means that if we define

$$
\bar{u}(t)=v(-t), \quad t \leq 0,
$$

we can conclude our proof, as $\bar{u} \in W^{1,2}(-\infty, 0) \cap \mathcal{X}_{\phi}$ and $\bar{u}^{\prime}(t)-\mathrm{A} \bar{u}(t)+B(\bar{u}(t), \bar{u}(t))=0$.

We have already mentioned in the Introduction that a finite dimensional counterpart of Theorem (17.1) was first derived in Theorem IV.3.1 in the monograph [20]. It has later been discussed in Example B.2 for finite dimensional Landau-Lifshitz-Gilbert equations by Kohn et al [28].

\section{Convergence of $U_{\delta}$ TO $U$}

Our aim in this section is to prove Theorem 8.3 , that is

$$
\lim _{\delta \rightarrow 0} U_{\delta}(\phi)=U(\phi), \quad \phi \in D\left(\mathrm{~A}^{\frac{\beta+1}{2}}\right) .
$$


To this purpose, we introduce an auxiliary functional $\tilde{S}_{-\infty}: \mathcal{X}_{\phi} \rightarrow[0, \infty]$, where $\phi \in D\left(\mathrm{~A}^{\frac{\beta+1}{2}}\right)$ is fixed, by the formula

$$
\tilde{S}_{-\infty}(v):= \begin{cases}S_{-\infty}(v), & \text { if } v \in \mathcal{X}_{\phi} \cap W^{1,2}\left(-\infty, 0 ; D\left(\mathrm{~A}^{\frac{\beta}{2}+1}\right), D\left(\mathrm{~A}^{\frac{\beta}{2}}\right)\right), \\ +\infty, & \text { if } v \in \mathcal{X}_{\phi} \backslash W^{1,2}\left(-\infty, 0 ; D\left(\mathrm{~A}^{\frac{\beta}{2}+1}\right), D\left(\mathrm{~A}^{\frac{\beta}{2}}\right)\right) .\end{cases}
$$

Lemma 8.1. Under Assumption 5.1, if $\phi \in \mathrm{H}$, then

$$
\Gamma-\lim _{\delta \rightarrow 0} S_{-\infty}^{\delta}=\mathrm{sc}^{-} \tilde{S}_{-\infty} \text { in } \mathcal{X}_{\phi}
$$

Proof. According to Proposition 4.7, the proof of (8.2) follows, once we show that for any $u \in \mathcal{X}_{\phi}$ the function

is decreasing and

$$
(0,1] \ni \delta \mapsto S_{-\infty}^{\delta}(u)
$$

$$
\lim _{\delta \rightarrow 0} S_{-\infty}^{\delta}(u)=\tilde{S}_{-\infty}(u), \quad u \in \mathcal{X}_{\phi}
$$

Let us fix a function $u \in \mathcal{X}_{\phi}$. In view of Assumption 5.1, for each $y \in D\left(A^{\frac{\beta}{2}}\right)$, the function $(0,1] \ni \delta \mapsto\left|Q_{\delta}^{-1} y\right|_{\mathrm{H}}^{2} \in \mathbb{R}$ is decreasing. This implies that for any fixed $u$ the mapping $(0,1] \ni \delta \mapsto S_{-\infty}^{\delta}(u)$ is decreasing.

We notice that if $u \in \mathcal{X}_{\phi} \backslash W^{1,2}\left(-\infty, 0 ; D\left(\mathrm{~A}^{\frac{\beta}{2}+1}\right), D\left(\mathrm{~A}^{\frac{\beta}{2}}\right)\right)$, then for any $\mathrm{d} \in(0,1)$

$$
S_{-\infty}^{\delta}(u)=\tilde{S}_{-\infty}(u)=+\infty,
$$

so that (8.3) follows. On the other end, if $u \in \mathcal{X}_{\phi} \cap W^{1,2}\left(-\infty, 0 ; D\left(\mathrm{~A}^{\frac{\beta}{2}+1}\right), D\left(\mathrm{~A}^{\frac{\beta}{2}}\right)\right)$, we have

$$
\tilde{S}_{-\infty}(u)=S_{-\infty}(u)=\frac{1}{2} \int_{-\infty}^{0}|\mathcal{H}(u)(t)|_{\mathrm{H}}^{2} d t .
$$

Thus, since $S_{-\infty}^{\delta}(u)=\frac{1}{2} \int_{-\infty}^{0}\left|Q_{\delta}^{-1} \mathcal{H}(u)(t)\right|_{\mathrm{H}}^{2} d t$, by the Lebesgue dominated convergence theorem we obtain (8.3), once we have observed that according to Assumption 5.1, for all $y \in D\left(\mathrm{~A}^{\frac{\beta}{2}}\right)$ it holds $Q_{\delta}^{-1} y \rightarrow y$, as $\delta \searrow 0$, and $\left|Q_{\delta}^{-1} y\right|_{\mathrm{H}} \leq\left|Q_{1}^{-1} y\right|_{\mathrm{H}}$.

Lemma 8.2. If $\phi \in \mathrm{V}$, then

$$
\mathrm{sc}^{-} \tilde{S}_{-\infty}(u)=S_{-\infty}(u), \quad u \in \mathcal{X}_{\phi} .
$$

Proof. In view of Proposition 4.5, we get (8.4) if we show that for every sequence $\left\{u_{n}\right\}_{n} \subset \mathcal{X}_{\phi}$ convergent to $u$ in $\mathcal{X}_{\phi}$ it holds

$$
S_{-\infty}(u) \leq \lim _{n} \inf \tilde{S}_{-\infty}\left(u_{n}\right),
$$

and for some sequence $\left\{u_{n}\right\}_{n} \subset \mathcal{X}_{\phi}$ convergent to $u$ in $\mathcal{X}_{\phi}$ it holds

$$
S_{-\infty}(u) \geq \limsup _{n} \tilde{S}_{-\infty}\left(u_{n}\right) .
$$


It is immediate to check that (8.5) follows from the lower semi-continuiuty of $S_{-\infty}$ and the definition of $\tilde{S}_{-\infty}$. Thus, let us prove (8.6). We are going to prove that there exists a sequence $\left\{u_{n}\right\}$ in $\mathcal{X}_{\phi} \cap W^{1,2}\left(-\infty, 0 ; D\left(\mathrm{~A}^{\frac{\beta}{2}+1}\right), D\left(\mathrm{~A}^{\frac{\beta}{2}}\right)\right)$ such that

$$
\lim _{n \rightarrow \infty} \sup _{t \in(-\infty, 0]}\left|u_{n}(t)-u(t)\right|_{\mathrm{H}}=0,
$$

and

$$
S_{-\infty}(u) \geq \limsup _{n \rightarrow \infty} \tilde{S}_{-\infty}\left(u_{n}\right) .
$$

To this purpose, we can assume that $S_{-\infty}(u)<\infty$. Then, according to Proposition A.1, we have that $u \in \mathcal{X}_{\phi} \cap W^{1,2}(-\infty, 0)$ and $\phi=u(0) \in \mathrm{V}$. Since

$$
W^{1,2}(-\infty, 0) \hookrightarrow C_{b}((-\infty, 0], \mathrm{H})
$$

it is enough to find a sequence $\left\{u_{n}\right\}$ in $\mathcal{X}_{\phi} \cap W^{1,2}\left(-\infty, 0 ; D\left(\mathrm{~A}^{\frac{\beta}{2}+1}\right), D\left(\mathrm{~A}^{\frac{\beta}{2}}\right)\right)$ satisfying (8.8) and, instead of (8.7), the following stronger condition

$$
\lim _{n \rightarrow \infty}\left|u_{n}-u\right|_{W^{1,2}(-\infty, 0)}=0 \text {. }
$$

Actually, if we have found a sequence $\left\{u_{n}\right\} \subset \mathcal{X}_{\phi} \cap W^{1,2}\left(-\infty, 0 ; D\left(\mathrm{~A}^{\frac{\beta}{2}+1}\right), D\left(\mathrm{~A}^{\frac{\beta}{2}}\right)\right)$ satisfying (8.9), then in view of (8.1), $\tilde{S}_{-\infty}\left(u_{n}\right)=S_{-\infty}\left(u_{n}\right)$ for every $n$. Therefore, in view of (8.9), we obtain (8.8), as $S_{-\infty}$ is a continuous functional on $W^{1,2}(-\infty, 0)$. Let us finally observe that the existence of the required sequence is just a consequence of the density of the space $\mathcal{X}_{\phi} \cap W^{1,2}\left(-\infty, 0 ; D\left(\mathrm{~A}^{\frac{1+\beta}{2}}\right), D\left(\mathrm{~A}^{\frac{\beta}{2}}\right)\right)$ in $\mathcal{X}_{\phi} \cap W^{1,2}(-\infty, 0)$.

Thus we can conclude that the following limit holds.

Theorem 8.3. Under Assumption 5.1, we have

$$
\lim _{\delta \rightarrow 0} U_{\delta}(\phi)=U(\phi), \quad \phi \in D\left(\mathrm{~A}^{\frac{\beta+1}{2}}\right) .
$$

Proof. Let us fix $\phi \in D\left(\mathrm{~A}^{\frac{\beta+1}{2}}\right)$. In view of Theorem 6.2

$$
U(\phi)=\min \left\{S_{-\infty}(u): u \in \mathcal{X}_{\phi}\right\} .
$$

and for any $\delta \in(0,1]$

$$
U_{\delta}(\phi)=\min \left\{S_{-\infty}^{\delta}(u): u \in \mathcal{X}_{\phi}\right\}
$$

Thus, thanks to Theorem 4.8, our result is proved as we have shown that for any $\phi \in D\left(\mathrm{~A}^{\frac{\beta+1}{2}}\right)$ the family $\left\{S_{-\infty}^{\delta}\right\}_{\delta \in(0,1]}$ is equi-coercive in $\mathcal{X}_{\phi}$ and, as a consequence of Lemma 8.1 and Lemma 8.2 ,

$$
\Gamma-\lim _{\delta \rightarrow 0} S_{-\infty}^{\delta}=S_{-\infty}, \quad \text { in } \mathcal{X}_{\phi}
$$




\section{Application to the exit problem}

A domain $D \subset \mathrm{H}$ is said to be invariant and attracted to the asymptotically stable equilibrium 0 of the deterministic Navier-Stokes equations (3.1)

$$
u^{\prime}(t)+A u(t)+B(u(t), u(t))=0, \quad u(0)=\phi,
$$

if, for any $\phi \in D$, the solution $u_{\phi}(t)$ to (9.1) remains in $D$, for every $t \geq 0$, and

$$
\lim _{t \rightarrow \infty}\left|u_{\phi}(t)\right|_{\mathrm{H}}=0 \text {. }
$$

It is well known that, as the solution $u_{\phi}$ satisfies inequality (3.2) by the Poincaré inequality (2.11), every ball in $\mathrm{H}$ is invariant and attracted to 0 .

Throughout this section, we will assume the following conditions on $D$.

Assumption 9.1. The set $D \subset \mathrm{H}$ is bounded, open, connected, contains 0 , is invariant and attracted to 0 . Moreover, for any $\phi \in \partial D \cap D\left(\mathrm{~A}^{\frac{1+\beta}{2}}\right)$ there exists a sequence $\left\{\phi_{n}\right\} \subset$ $(\mathrm{H} \backslash \bar{D}) \cap D\left(\mathrm{~A}^{\frac{1+\beta}{2}}\right)$ such that

$$
\lim _{n \rightarrow \infty}\left|\phi_{n}-\phi\right|_{D\left(\mathrm{~A} \frac{1+\beta}{2}\right)}=0 .
$$

Remark 9.2. If for every $\phi \in \partial D \cap D\left(\mathrm{~A}^{\frac{1+\beta}{2}}\right)$ there exists $y \in \mathrm{H} \backslash \bar{D} \cap D\left(\mathrm{~A}^{\frac{1+\beta}{2}}\right)$ such that

$$
\{t \phi+(1-t) y: t \in[0,1)\} \subset \mathrm{H} \backslash \bar{D},
$$

then Assumption 9.1 is clearly satisfied. Such a property is true if, for example, $D$ is convex.

Lemma 9.3. For any $\delta \in(0,1]$, there exists $y_{\delta} \in \partial D$ such that

$$
\inf _{y \in \partial D} U_{\delta}(y)=U_{\delta}\left(y_{\delta}\right)
$$

Proof. First we will show that

$$
\inf _{\phi \in \partial D} U_{\delta}(\phi)<\infty .
$$

Since $\mathrm{H} \backslash \bar{D}$ is an open subset of $\mathrm{H}$ and the space $D\left(\mathrm{~A}^{\frac{1+\beta}{2}}\right)$ is dense in $\mathrm{H}$, there exists $\tilde{\phi} \in(\mathrm{H} \backslash \bar{D}) \cap D\left(\mathrm{~A}^{\frac{1+\beta}{2}}\right)$. Since $0 \in D$, and the path $t \mapsto t \tilde{\phi}$ is continuous, there must exist $0<t_{0}<1$ such that $t_{0} \tilde{\phi} \in \partial D$. Clearly, $t_{0} \tilde{\phi} \in D\left(\mathrm{~A}^{\frac{1+\beta}{2}}\right)$, so that, as $\partial D \cap D\left(\mathrm{~A}^{\frac{1+\beta}{2}}\right) \neq \emptyset$, according to Proposition 6.1, property (9.3) follows.

Due to the compactness of the level sets of the functionals $U_{\delta}$, we infer that there exists $y_{\delta} \in \partial D \cap D\left(\mathrm{~A}^{\frac{1+\beta}{2}}\right)$ such that (9.2) holds.

Now, for any $\phi \in D, \varepsilon>0$ and $\delta \in(0,1]$, we will denote by $\tau_{\phi}^{\varepsilon, \delta}$ the exit time of the solution $u_{\phi}^{\varepsilon, \delta}$ of equation (5.1) from the domain $D$, that is

$$
\tau_{\phi}^{\varepsilon, \delta}=\inf \left\{t \geq 0: u_{\phi}^{\varepsilon, \delta}(t) \in \partial D\right\} .
$$

Our purpose here is to prove the following exponential estimate for the expectation of $\tau_{\phi}^{\varepsilon, \delta}$ in terms of the infimum of $U_{\delta}$ on the boundary of $D$. 
Theorem 9.4. For any $\delta \in(0,1]$ and $\phi \in D$

$$
\lim _{\varepsilon \rightarrow 0} \varepsilon \log \mathbb{E} \tau_{\phi}^{\varepsilon, \delta}=\min _{y \in \partial D} U_{\delta}(y) .
$$

As we already pointed out in [11, Section 7], the proof of the previous result is based on the few lemmas below, whose proofs are postponed till Appendix B. Actually, the arguments used in finite dimension (see [19, proof of Theorem 5.7.11] and [20, proof of Theorem 4.1]), can be adapted to this infinite dimensional case, once the following preliminary results are proven.

Lemma 9.5. For any $\eta>0$ and $\mu>0$, there exist $T_{0}=T_{0}(\eta, \mu)>0$ and $h=h(\eta)>0$ such that for all $\phi \in B_{0}(\mu)$ there exist $T \leq T_{0}$ and $v \in C([0, T] ; \mathrm{H})$, with $v(0)=\phi$, such that

$$
d_{\mathrm{H}}(v(T), \bar{D})=h
$$

and

$$
S_{0, T}^{\delta}(v) \leq \inf _{y \in \partial D} U_{\delta}(y)+\eta
$$

Lemma 9.6. There exists $\mu_{0}>0$ such that for any $\eta>0$ and $\mu \in\left(0, \mu_{0}\right]$

$$
\lim _{\varepsilon \rightarrow 0} \varepsilon \log \left(\inf _{\phi \in B_{0}(\mu)} \mathbb{P}\left(\tau_{\phi}^{\varepsilon, \delta} \leq T\right)\right)>-\left(\inf _{y \in \partial D} U_{\delta}(y)+\eta\right),
$$

for some $T=T(\eta, \mu)>0$.

Lemma 9.7. For any $\mu>0$ such that $B_{0}(\mu) \subset D$,

$$
\lim _{t \rightarrow+\infty} \liminf _{\varepsilon \rightarrow 0} \varepsilon \log \left(\sup _{\phi \in D} \mathbb{P}\left(\sigma_{\phi}^{\varepsilon, \delta, \mu}>t\right)\right)=-\infty,
$$

where

Moreover,

$$
\sigma_{\phi}^{\varepsilon, \delta, \mu}:=\inf \left\{t \geq 0 ; u_{\phi}^{\varepsilon, \delta}(t) \in B_{0}(\mu) \cup \partial D\right\}
$$

$$
\lim _{\varepsilon \rightarrow 0} \mathbb{P}\left(u_{\phi}^{\varepsilon, \delta}\left(\sigma_{\phi}^{\varepsilon, \delta, \mu}\right) \in B_{0}(\mu)\right)=1
$$

Lemma 9.8. For any closed set $N \subset \partial D$,

$$
\lim _{\mu \rightarrow 0} \limsup _{\varepsilon \rightarrow 0} \varepsilon \log \left(\sup _{\phi \in B_{0}(3 \mu)} \mathbb{P}\left(u_{\phi}^{\varepsilon, \delta}\left(\sigma_{\phi}^{\varepsilon, \delta, \mu}\right) \in N\right)\right) \leq-\inf _{\phi \in N} U_{\delta}(\phi) .
$$

Lemma 9.9. For every $\lambda>0$ and $\mu>0$ such that $B_{0}(\mu) \subset D$, there exists $T=T(\mu, \lambda)<\infty$ such that

$$
\limsup _{\varepsilon \rightarrow 0} \varepsilon \log \left(\sup _{\phi \in B_{0}(\mu)} \mathbb{P}\left(\sup _{t \in[0, T]}\left|u_{\phi}^{\varepsilon, \delta}(t)-\phi\right|_{\mathrm{H}} \geq 3 \mu\right)\right)<-\lambda .
$$


Next, by proceeding as in the proof of [11, Theorem 7.7]) we can conclude that the following approximation result holds.

Theorem 9.10. Suppose that Assumption 5.1 is satisfied. If for any $\phi \in \mathrm{V} \cap \partial D$ there exists a sequence $\left\{\phi_{n}\right\} \subset D\left(\mathrm{~A}^{\frac{1+\beta}{2}}\right) \cap \partial D$ such that

$$
\lim _{n \rightarrow \infty}\left|\phi_{n}-\phi\right|_{\mathrm{V}}=0
$$

then

$$
\lim _{\delta \rightarrow 0} \inf _{\phi \in \partial D} U_{\delta}(\phi)=\inf _{\phi \in \partial D} U(\phi) .
$$

Sketch of the Proof. Limit (9.8) follows from Theorem 6.2 and (9.7) in virtue of a general argument based on $\Gamma$-convergence and relaxation, which applies to more general situations, and which has been introduced in [11. Actually, we define

$$
\tilde{U}(\phi)= \begin{cases}U(\phi), & \phi \in \Lambda_{\beta}, \\ +\infty, & \phi \in H \backslash \Lambda_{\beta},\end{cases}
$$

and for any $\delta \in(0,1]$

$$
\tilde{U}_{\delta}(\phi)= \begin{cases}U_{\delta}(\phi), & \phi \in \Lambda_{\beta}, \\ +\infty, & \phi \in H \backslash \Lambda_{\beta},\end{cases}
$$

where $\Lambda_{\beta}=D\left(A^{\frac{1+\beta}{2}}\right) \cap \partial D$. One can prove that

$$
\Gamma-\lim _{\delta \rightarrow 0} \tilde{U}_{\delta}=\mathrm{sc}^{-} \tilde{U}, \quad \text { in } \mathrm{H},
$$

and then, by using (9.7) and the continuity of $U$ in the space $\mathrm{V}$ proved in Proposition 6.6, one can show

$$
\mathrm{sc}^{-} \tilde{U}(\phi)= \begin{cases}U(\phi), & \phi \in \partial D \\ +\infty, & \phi \in \mathrm{H} \backslash \partial D\end{cases}
$$

This implies (9.8).

In view of Theorems 9.4 and 9.10, we obtain the following result.

Corollary 9.11. Under the same assumptions of Theorem 9.10, we have

$$
\lim _{\delta \rightarrow 0} \lim _{\varepsilon \rightarrow 0} \varepsilon \log \mathbb{E} \tau_{\phi}^{\varepsilon, \delta}=\inf _{\phi \in \partial D} U(\phi) .
$$

Informally, this means that for $0<\varepsilon<<\delta<<1$, the following asymptotic formula holds

$$
\mathbb{E} \tau_{\phi}^{\varepsilon, \delta} \sim \exp \left(\frac{1}{\varepsilon} \inf _{\phi \in \partial D} U(\phi)\right) .
$$

Remark 4. As in [11, Remark 7.8], we notice that if we take $D=B_{0}(r)$, for $r>0$, then condition (9.7) assumed in Theorem 9.10 is fulfilled. Actually, as $D\left(A^{\frac{1+\beta}{2}}\right)$ is dense in $\mathrm{V}$, we can find a sequence $\left\{\hat{\phi}_{n}\right\} \subset D\left(A^{\frac{1+\beta}{2}}\right)$ which is convergent to $\phi$ in $\mathrm{V}$. Then, if we set $\phi_{n}=r \hat{\phi}_{n} /\left|\hat{\phi}_{n}\right|_{\mathrm{H}}$, we conclude that $\left\{\phi_{n}\right\} \subset D\left(\mathrm{~A}^{\frac{1+\beta}{2}}\right) \cap \partial D$ and (9.7) holds. 


\section{Appendix A. Proofs of some Auxiliary Results}

Proposition A.1. Assume that $z \in \mathcal{X}$ is such that $S_{-\infty}(z)<\infty$. Then, $z(0) \in V$,

$$
\lim _{t \rightarrow-\infty}|z(t)|_{\mathrm{V}}=0
$$

and $z \in W^{1,2}(-\infty, 0)$, i.e.

$$
\int_{-\infty}^{0}|A z(t)|_{\mathrm{H}}^{2} d t+\int_{-\infty}^{0}\left|z^{\prime}(t)\right|_{\mathrm{H}}^{2} d t<\infty
$$

Moreover, there exists a continuous and strictly increasing function $\varphi:[0, \infty) \rightarrow[0, \infty)$ such that $\varphi(0)=0$ and, if $z \in \mathcal{X}$ is a solution to the problem

$$
z^{\prime}(t)+\mathrm{A} z(t)+\mathrm{B}(z(t), z(t))=f(t), \quad t \leq 0,
$$

with $f$ being an element of $L^{2}(-\infty, 0)$, then $z \in W^{1,2}(-\infty, 0), z(0) \in V$ and

$$
|z(0)|_{\mathrm{V}}^{2}+|z|_{W^{1,2}(-\infty, 0)}^{2} \leq \varphi\left(\int_{-\infty}^{0}|f(t)|_{\mathrm{H}}^{2} d t\right) .
$$

Proof. The argument below is a bit informal but it can easily be made fully rigorous. We will be careful with the constants as we want to prove the last part of the Proposition as well. We have that $z(0) \in V$, as a consquence of Lemma 3.8. Next, we will be proving (A.1). In view of Lemma 3.8, we can assume that $z \in W_{\text {loc }}^{1,2}(-\infty, 0)$. Since $S_{-\infty}(z)<\infty$, if we set

$$
f(t)=z^{\prime}(t)+A z(t)+B(z(t), z(t)), \quad t \leq 0,
$$

we have that $f \in L^{2}(-\infty, 0 ; H)$. If we multiply equation (A.3) by $z$ and use equality (2.16), we get

$$
\frac{1}{2} \frac{d}{d t}|z(t)|_{\mathrm{H}}^{2}+|z(t)|_{\mathrm{V}}^{2}=(f, z)_{\mathrm{H}} \leq \frac{1}{2}|z(t)|_{\mathrm{V}}^{2}+\frac{1}{2 \lambda_{1}}|f(t)|_{\mathrm{H}}^{2}, \quad t<0
$$

where $\lambda_{1}$ is the Poincare constant of the domain $\mathcal{O}$. Hence,

$$
|z(t)|_{\mathrm{H}}^{2}+\int_{s}^{t}|z(r)|_{\mathrm{V}}^{2} d r \leq|z(s)|_{\mathrm{H}}^{2}+\frac{1}{\lambda_{1}} \int_{s}^{t}|f(r)|_{\mathrm{H}}^{2} d r, \quad-\infty<s \leq t \leq 0 .
$$

As

$$
\lim _{s \rightarrow-\infty}|z(s)|_{\mathrm{H}}=0
$$

we infer that

$$
|z(t)|_{\mathrm{H}}^{2}+\int_{-\infty}^{t}|z(s)|_{\mathrm{V}}^{2} d s \leq \frac{1}{\lambda_{1}} \int_{-\infty}^{t}|f(r)|_{\mathrm{H}}^{2} d r,-\infty<t \leq 0 .
$$

This implies

$$
|z(t)|_{\mathrm{H}}^{2} \leq \frac{1}{\lambda_{1}} \int_{-\infty}^{t}|f(r)|_{\mathrm{H}}^{2} d r \leq \frac{1}{\lambda_{1}} \int_{-\infty}^{0}|f(r)|_{\mathrm{H}}^{2} d r, \quad t \leq 0
$$

and

$$
\int_{-\infty}^{0}|z(s)|_{\mathrm{V}}^{2} d s \leq \frac{1}{\lambda_{1}} \int_{-\infty}^{0}|f(r)|_{\mathrm{H}}^{2} d r
$$


The latter inequality means that $z \in L^{2}((-\infty, 0], V)$, which implies that we can find a decreasing sequence $\left\{s_{n}\right\}$ such that $s_{n} \searrow-\infty$ and

$$
\lim _{n \rightarrow \infty}\left|z\left(s_{n}\right)\right|_{\mathrm{V}}=0 \text {. }
$$

Next we multiply equation (A.3) by $\mathrm{A} z(t)$. Thanks to (2.15) and to the Young inequality, we get

$$
\begin{aligned}
\frac{1}{2} \frac{d}{d t}|z(t)|_{\mathrm{V}}^{2}+|\mathrm{A} z(t)|_{\mathrm{H}}^{2} & =-(B(z(t), z(t)), A z(t))_{\mathrm{H}}+(f(t), \mathrm{A} z(t))_{\mathrm{H}} \\
& \leq \frac{1}{4}|\mathrm{~A} z(t)|_{\mathrm{H}}^{2}+\frac{C_{2}}{2}|z(t)|_{\mathrm{H}}^{2}|z(t)|_{\mathrm{V}}^{4}+\frac{1}{4}|\mathrm{~A} z(t)|_{\mathrm{H}}^{2}+|f(t)|_{\mathrm{H}}^{2} .
\end{aligned}
$$

where $C_{2}=\frac{54}{4} C^{2}$ and $C$ is the constant from inequality (2.15).

Applying next the Poincaré inequality (2.1) we get,

$$
\frac{d}{d t}|z(t)|_{\mathrm{V}}^{2}+\lambda_{1}|z(t)|_{\mathrm{V}}^{2} \leq C_{2}\left[|z(t)|_{\mathrm{H}}^{2}|z(t)|_{\mathrm{V}}^{2}\right]|z(t)|_{\mathrm{V}}^{2}+2|f(t)|_{\mathrm{H}}^{2}
$$

Hence, since $\lambda_{1} \geq 0$, we have

$$
\frac{d}{d t}|z(t)|_{\mathrm{V}}^{2} \leq C_{2}\left[|z(t)|_{\mathrm{H}}^{2}|z(t)|_{\mathrm{H}}^{2}\right]|z(t)|_{\mathrm{V}}^{2}+2|f(t)|_{\mathrm{H}}^{2}
$$

and so, by the Gronwall Lemma, for any $-\infty<s \leq t \leq 0$ we get

$$
\begin{aligned}
|z(t)|_{\mathrm{V}}^{2} & \leq|z(s)|_{\mathrm{V}}^{2} \exp \left(C_{2} \int_{s}^{t}|z(r)|_{\mathrm{H}}^{2}|z(r)|_{\mathrm{V}}^{2} d r\right) \\
& +2 \int_{s}^{t}|f(r)|_{\mathrm{H}}^{2} \exp \left(C_{2} \int_{r}^{t}|z(\rho)|_{\mathrm{H}}^{2}|z(\rho)|_{\mathrm{V}}^{2} d \rho\right) d r
\end{aligned}
$$

Using the above inequality with $s=s_{n}$ from (A.7) and then taking the limit as $n \rightarrow \infty$, we infer that

$$
|z(t)|_{\mathrm{V}}^{2} \leq 2 \int_{-\infty}^{t}|f(r)|_{\mathrm{H}}^{2} \exp \left(C_{2} \int_{r}^{t}|z(\rho)|_{\mathrm{H}}^{2}|z(\rho)|_{\mathrm{V}}^{2} d \rho\right) d r, \quad t \leq 0 .
$$

Of course, for the above to be correct we need to show that the sequence

$$
\left\{\int_{s_{n}}^{t}|z(r)|_{\mathrm{H}}^{2}|z(r)|_{\mathrm{V}}^{2} d r\right\}_{n \geq 1}
$$

is bounded from above. But in view of estimates (A.5) and (A.6) we have

$$
\int_{-\infty}^{0}|z(\rho)|_{\mathrm{H}}^{2}|z(\rho)|_{\mathrm{V}}^{2} d \rho \leq \frac{1}{\lambda_{1}^{2}}|f|_{L^{2}(-\infty, 0, \mathrm{H})}^{4}<\infty
$$

Therefore, since

$$
\int_{r}^{t}|z(\rho)|_{\mathrm{H}}^{2}|z(\rho)|_{\mathrm{V}}^{2} d \rho \leq \int_{-\infty}^{0}|z(\rho)|_{\mathrm{H}}^{2}|z(\rho)|_{\mathrm{V}}^{2} d \rho, \quad-\infty<r \leq t \leq 0
$$


we can conclude that

$$
\sup _{t \leq 0}|z(t)|_{\mathrm{V}}^{2} \leq 2 \exp \left(\frac{C_{2}}{\lambda_{1}^{2}}|f|^{4}\right) \sup _{t \leq 0} \int_{-\infty}^{t}|f(r)|_{\mathrm{H}}^{2} d r \leq 2 \exp \left(\frac{C_{2}}{\lambda_{1}^{2}}|f|^{4}\right)|f|^{2}
$$

(here, for the sake of brevity, we denote $|f|_{L^{2}(-\infty, 0 ; H)}=|f|$ ).

Moreover, as

$$
\int_{-\infty}^{0}|f(r)|_{\mathrm{H}}^{2} \exp \left(C_{2} \int_{r}^{t}|z(\rho)|_{\mathrm{H}}^{2}|z(\rho)|_{\mathrm{V}}^{2} d \rho\right) d r<\infty
$$

we have

$$
\lim _{t \rightarrow-\infty} \int_{-\infty}^{t}|f(r)|_{\mathrm{H}}^{2} \exp \left(C_{2} \int_{r}^{t}|z(\rho)|_{\mathrm{H}}^{2}|z(\rho)|_{\mathrm{V}}^{2} d \rho\right) d r=0
$$

so that from (A.12) we conclude that (A.1) holds.

Now, to prove that $z \in W^{1,2}(-\infty, 0)$, we observe that from (A.8) we also have

$$
|z(0)|_{\mathrm{V}}^{2}+\int_{-\infty}^{0}|A z(t)|_{\mathrm{H}}^{2} d t \leq C_{2} \int_{-\infty}^{0}\left[|z(t)|_{\mathrm{H}}^{2}|z(t)|_{\mathrm{V}}^{2}\right]|z(t)|_{\mathrm{V}}^{2} d t+2 \int_{-\infty}^{0}|f(t)|_{\mathrm{H}}^{2} d t
$$

where we have used (A.1). Since by (A.5) and (A.14),

$$
\sup _{t \in(-\infty, 0]}|z(t)|_{\mathrm{H}}^{2}|z(t)|_{\mathrm{V}}^{2} \leq \frac{2}{\lambda_{1}} \exp \left(\frac{C_{2}}{\lambda_{1}^{2}}|f|^{4}\right)|f|^{4}<\infty
$$

we infer that

$$
|z(0)|_{\mathrm{V}}^{2}+\int_{-\infty}^{0}|A z(t)|_{\mathrm{H}}^{2} d t \leq \frac{2 C_{2}}{\lambda_{1}} \exp \left(\frac{C_{2}}{\lambda_{1}^{2}}|f|^{4}\right)|f|^{4} \int_{-\infty}^{0}|z(t)|_{\mathrm{V}}^{2} d t+2 \int_{-\infty}^{0}|f(t)|_{\mathrm{H}}^{2} d t
$$

Hence, in view of (A.6), we infer that

$$
|z(0)|_{\mathrm{V}}^{2}+\int_{-\infty}^{0}|A z(t)|_{\mathrm{H}}^{2} d t \leq \frac{2 C_{2}}{\lambda_{1}^{2}} \exp \left(\frac{C_{2}}{\lambda_{1}^{2}}|f|^{4}\right)|f|^{6}+2|f|^{2},
$$

and this concludes the proof of the first part of (A.2).

In order to prove the second part of (A.2), it is enough to show that

$$
\int_{-\infty}^{0}|B(z(t), z(t))|_{\mathrm{H}}^{2} d t<\infty
$$

Indeed, by the Minkowski inequality we have

$$
\left|z^{\prime}\right|_{L^{2}(-\infty, 0 ; \mathrm{H})} \leq|A z|_{L^{2}(-\infty, 0 ; \mathrm{H})}+|B(z, z)|_{L^{2}(-\infty, 0 ; \mathrm{H})}+|f|_{L^{2}(-\infty, 0 ; \mathrm{H})} .
$$


According to inequalities (A.5), (A.12) and (A.16) and to inequality (2.15), we have

$$
\begin{aligned}
& \int_{-\infty}^{0}|B(z(t), z(t))|_{\mathrm{H}}^{2} d t \leq C \int_{-\infty}^{0}|z(t)|_{\mathrm{H}}|z(t)|_{\mathrm{V}}^{2}|A z(t)|_{\mathrm{H}} d t \\
& \leq C \sup _{t \leq 0}|z(t)|_{\mathrm{H}}|z(t)|_{\mathrm{V}}\left(\int_{-\infty}^{0}|z(t)|_{\mathrm{V}}^{2} d t\right)^{\frac{1}{2}}\left(\int_{-\infty}^{0}|A z(t)|_{\mathrm{H}}^{2} d t\right)^{\frac{1}{2}} \\
& \leq \frac{2}{\lambda_{1}} \exp \left(\frac{C_{2}}{\lambda_{1}^{2}}|f|^{4}\right)|f|^{4} \frac{1}{\sqrt{\lambda_{1}}}|f|\left(\frac{2 C_{2}}{\lambda_{1}^{2}} \exp \left(\frac{C_{2}}{\lambda_{1}^{2}}|f|^{4}\right)|f|^{6}+2|f|^{2}\right)^{\frac{1}{2}}<\infty
\end{aligned}
$$

The final statement follows from inequalities (A.16), (A.17) and (A.18).

Remark 5. (1) Our proof of Proposition A.1 has been inspired by [9].

(2) Roughly speaking, the above result says that the following two equalities hold

$$
\left\{z \in \mathcal{X}: S_{-\infty}(z)<\infty\right\}=\mathcal{X} \cap W^{1,2}(-\infty, 0)
$$

and

$$
\left\{z \in \mathcal{X}: u(0)=\phi \text { and } S_{-\infty}(z)<\infty\right\}=\mathcal{X}_{\phi} \cap W^{1,2}(-\infty, 0), \quad \phi \in \mathrm{V} .
$$

Next Proposition generalizes Proposition $\mathrm{A} .1$ to $S_{-\infty}^{\delta}$.

Proposition A.2. Assume that $\alpha \in(0,1 / 2)$ and $z \in \mathcal{X}$ is such that $S_{-\infty}^{\delta}(z)<\infty$. Let $f \in L^{2}\left((-\infty, 0] ; D\left(\mathrm{~A}^{\frac{\alpha}{2}}\right)\right.$ be defined as

$$
z^{\prime}(t)+\mathrm{A} z(t)+\mathrm{B}(z(t), z(t))=f(t), \quad t \leq 0 .
$$

Then $z(0) \in D\left(\mathrm{~A}^{\frac{\alpha+1}{2}}\right)$,

$$
\lim _{t \rightarrow-\infty}|z(t)|_{D\left(\mathrm{~A} \frac{\alpha+1}{2}\right)}=0
$$

and $z \in W^{1,2}\left(-\infty, 0 ; D\left(\mathrm{~A}^{1+\frac{\alpha}{2}}\right), D\left(\mathrm{~A}^{\frac{\alpha}{2}}\right)\right)$, i.e.

$$
\int_{-\infty}^{0}\left|A^{\frac{\alpha}{2}+1} z(t)\right|_{\mathrm{H}}^{2} d t+\int_{-\infty}^{0}\left|A^{\frac{\alpha+1}{2}} z^{\prime}(t)\right|_{\mathrm{H}}^{2} d t<\infty .
$$

Moreover, there exists a continuous and strictly increasing function $\varphi:[0, \infty) \rightarrow[0, \infty)$ such that $\varphi(0)=0$ and if $z \in \mathcal{X}$ is a solution to the problem

$$
z^{\prime}(t)+\mathrm{A} z(t)+\mathrm{B}(z(t), z(t))=f(t), \quad t \leq 0,
$$

with $f$ being an element of $L^{2}\left(-\infty, 0 ; D\left(\mathrm{~A}^{\frac{\alpha}{2}}\right)\right)$, then $z \in W^{1,2}\left(-\infty, 0 ; D\left(\mathrm{~A}^{\frac{\alpha}{2}+1}\right), D\left(\mathrm{~A}^{\frac{\alpha}{2}}\right)\right)$, $z(0) \in D\left(\mathrm{~A}^{\frac{\alpha+1}{2}}\right)$ and

$$
|z(0)|_{D\left(\mathrm{~A} \frac{\alpha+1}{2}\right)}^{2}+|z|_{W^{1,2}\left(-\infty, 0 ; D\left(\mathrm{~A}^{\frac{\alpha}{2}+1}\right), D\left(\mathrm{~A}^{\frac{\alpha}{2}}\right)\right)}^{2} \leq \varphi\left(|f|_{L^{2}(-\infty, 0)}^{2}\right) .
$$


Proof. Following the methods from the proof of Proposition A.1 it is sufficient to prove the first part of Proposition A.2.

Let us fix $\alpha \in(0,1 / 2), \phi \in D\left(\mathrm{~A}^{\frac{1+\alpha}{2}}\right)$ and $z \in \mathcal{X}_{\phi}$ such that $S_{-\infty}^{\delta}(z)<\infty$. Let us define $f \in L^{2}\left(-\infty, 0 ; D\left(\mathrm{~A}^{\frac{\alpha}{2}}\right)\right.$ by $\left.\mathrm{A} .19\right)$. Since the assumptions in the present proposition are stronger than the assumptions of Proposition A.1, we can freely use the results from the proof of the latter.

So, firstly, let us notice that by inequality (A.15) we can find a decreasing sequence $\left\{s_{n}\right\}$ such that $s_{n} \downarrow-\infty$ and

$$
\lim _{n \rightarrow \infty}\left|\mathrm{A}^{\frac{1+\alpha}{2}} z\left(s_{n}\right)\right|_{\mathrm{H}}=0 .
$$

Arguing as in the proof of Proposition 3.3 , if we calculate the derivative of $\left|\mathrm{A}^{\frac{\alpha+1}{2}} u(t)\right|_{\mathrm{H}}^{2}$ and use inequality (2.20), with $s=2$, to get the following generalisation of (A.8)

$$
\begin{aligned}
\frac{1}{2} \frac{d}{d t}\left|\mathrm{~A}^{\frac{1+\alpha}{2}} z(t)\right|_{\mathrm{H}}^{2}+\left|\mathrm{A}^{\frac{\alpha}{2}+1} z(t)\right|_{\mathrm{H}}^{2} & =-\left(B(z(t), z(t)), \mathrm{A}^{\alpha+1} z(t)\right)_{\mathrm{H}}+\left(f(t), \mathrm{A}^{\alpha+1} z(t)\right)_{\mathrm{H}} \quad(\mathrm{A} .23) \\
& \leq \frac{1}{2}\left|\mathrm{~A}^{\frac{\alpha}{2}+1} z(t)\right|_{\mathrm{H}}^{2}+C|\mathrm{~A} z(t)|_{\mathrm{H}}^{2}\left|\mathrm{~A}^{\frac{\alpha}{2}+1} z(t)\right|^{2}+C\left|\mathrm{~A}^{\frac{\alpha}{2}} f(t)\right|_{\mathrm{H}}^{2} .
\end{aligned}
$$

Let us note that contrary to (A.8), the highest power of $z$ on the RHS of (A.23) is 4 . Hence, we infer that

$$
\frac{d}{d t}\left|\mathrm{~A}^{\frac{1+\alpha}{2}} z(t)\right|_{\mathrm{H}}^{2}+\left|\mathrm{A}^{\frac{\alpha}{2}+1} z(t)\right|_{\mathrm{H}}^{2} \leq C|\mathrm{~A} z(t)|_{\mathrm{H}}^{2}\left|\mathrm{~A}^{\frac{1+\alpha}{2}} z(t)\right|_{\mathrm{H}}^{2}+2\left|\mathrm{~A}^{\frac{\alpha}{2}} f(t)\right|_{\mathrm{H}}^{2} .
$$

Therefore, by the Gronwall Lemma, for any $-\infty<s \leq t \leq 0$ we get

$$
\begin{aligned}
\left|\mathrm{A}^{\frac{1+\alpha}{2}} z(t)\right|_{\mathrm{H}}^{2} & \leq\left|\mathrm{A}^{\frac{1+\alpha}{2}} z(s)\right|_{\mathrm{H}} \exp \left(C \int_{s}^{t}|\mathrm{~A} z(r)|_{\mathrm{H}}^{2} d r\right) \\
& +2 \int_{s}^{t}\left|\mathrm{~A}^{\frac{\alpha}{2}} f(r)\right|_{\mathrm{H}}^{2} \exp \left(C \int_{r}^{t}|\mathrm{~A} z(\rho)|_{\mathrm{H}}^{2} d \rho\right) d r .
\end{aligned}
$$

Using the above with $s=s_{n}$ from (A.22) and then taking the limit as $n \rightarrow \infty$, we infer that

$$
\left|\mathrm{A}^{\frac{1+\alpha}{2}} z(t)\right|_{\mathrm{H}}^{2} \leq 2 \int_{-\infty}^{t}\left|\mathrm{~A}^{\frac{\alpha}{2}} f(r)\right|_{\mathrm{H}}^{2} \exp \left(C \int_{r}^{t}|\mathrm{~A} z(\rho)|_{\mathrm{H}}^{2} d \rho\right) d r, \quad t \leq 0 .
$$

As in the proof of the previous Proposition A.1 the above is true because now by inequality (A.16) the sequence

$$
\left\{\int_{s_{n}}^{t}|\mathrm{~A} z(r)|_{\mathrm{H}}^{2} d r\right\}_{n \geq 1}
$$

is bounded from above by $\frac{2 C_{2}}{\lambda_{1}^{2}} \exp \left(\frac{C_{2}}{\lambda_{1}^{2}}|f|^{4}\right)|f|^{6}+2|f|^{2}$, where $|f|$ denotes $|f|_{L^{2}(-\infty, 0 ; \mathrm{H})}$. Therefore, we can conclude that

$$
\sup _{t \leq 0}\left|\mathrm{~A}^{\frac{1+\alpha}{2}} z(t)\right| \leq 2 \int_{-\infty}^{0}\left|\mathrm{~A}^{\frac{\alpha}{2}} f(r)\right|_{\mathrm{H}}^{2} d r \exp \left(C \frac{2 C_{2}}{\lambda_{1}^{2}} \exp \left(\frac{C_{2}}{\lambda_{1}^{2}}|f|^{4}\right)|f|^{6}+2|f|^{2}\right) .
$$


Moreover, as

$$
\int_{-\infty}^{0}\left|\mathrm{~A}^{\frac{\alpha}{2}} f(r)\right|_{\mathrm{H}}^{2} \exp \left(C \int_{r}^{t}|\mathrm{~A} z(\rho)|_{\mathrm{H}}^{2} d \rho\right) d r<\infty
$$

we have that

$$
\lim _{t \rightarrow-\infty} \int_{-\infty}^{t}\left|\mathrm{~A}^{\frac{\alpha}{2}} f(r)\right|_{\mathrm{H}}^{2} \exp \left(C \int_{r}^{t}|\mathrm{~A} z(\rho)|_{\mathrm{H}}^{2} d \rho\right) d r=0 .
$$

Hence A.20) follows from A.26).

Now, to prove the second part of Proposition A.2, i.e. the first inequality in (A.20), we observe that from (A.24) we also have

$$
\left|\mathrm{A}^{\frac{\alpha+1}{2}} z(0)\right|_{\mathrm{H}}^{2}+\int_{-\infty}^{0}\left|\mathrm{~A}^{\frac{\alpha+2}{2}} z(t)\right|_{\mathrm{H}}^{2} d t \leq C \int_{-\infty}^{0}\left[|\mathrm{~A} z(t)|_{\mathrm{H}}^{2}\right]\left|\mathrm{A}^{\frac{\alpha+1}{2}} z(t)\right|_{\mathrm{H}}^{2} d t+2 \int_{-\infty}^{0}\left|\mathrm{~A}^{\frac{\alpha}{2}} f(t)\right|_{\mathrm{H}}^{2} d t .
$$

Taking into account inequalities (A.27) and (A.16) we infer that

$$
\begin{aligned}
& \left|\mathrm{A}^{\frac{\alpha+1}{2}} z(0)\right|_{\mathrm{H}}^{2}+\int_{-\infty}^{0}\left|\mathrm{~A}^{\frac{\alpha+2}{2}} z(t)\right|_{\mathrm{H}}^{2} d t \leq C\left(\frac{2 C_{2}}{\lambda_{1}^{2}} \exp \left(\frac{C_{2}}{\lambda_{1}^{2}}|f|^{4}\right)|f|^{6}+2|f|^{2}\right) \\
& \left(\int_{-\infty}^{0}\left|\mathrm{~A}^{\frac{\alpha}{2}} f(r)\right|_{\mathrm{H}}^{2} d r \exp \left(C \frac{2 C_{2}}{\lambda_{1}^{2}} \exp \left(\frac{C_{2}}{\lambda_{1}^{2}}|f|^{4}\right)|f|^{6}+2|f|^{2}\right)\right)+2 \int_{-\infty}^{0}\left|\mathrm{~A}^{\frac{\alpha}{2}} f(t)\right|_{\mathrm{H}}^{2} d t
\end{aligned}
$$

and this concludes the proof of the first part of inequality (A.20).

As in the proof of the previous Proposition, in order to prove the third part of Proposition A.2, i.e. the second inequality in (A.20), it is enough to show that

$$
\int_{-\infty}^{0}\left|\mathrm{~A}^{\frac{\alpha}{2}} B(z(t), z(t))\right|_{\mathrm{H}}^{2} d t<\infty .
$$

According to inequalities (2.20) (with $s=2)$ ), (A.16) and (A.27) we have

$$
\begin{aligned}
\int_{-\infty}^{0}\left|\mathrm{~A}^{\frac{\alpha}{2}} B(z(t), z(t))\right|_{\mathrm{H}}^{2} d t \leq C \int_{-\infty}^{0}|\mathrm{~A} z(t)|_{\mathrm{H}}^{2}\left|\mathrm{~A}^{\frac{\alpha+1}{2}} z(t)\right|_{\mathrm{H}}^{2} d t \\
\leq C \sup _{t \leq 0}\left|\mathrm{~A}^{\frac{\alpha+1}{2}} z(t)\right|_{\mathrm{H}}^{2} \int_{-\infty}^{0}|\mathrm{~A} z(t)|_{\mathrm{H}}^{2} d t \leq\left(\frac{4 C C_{2}}{\lambda_{1}^{2}} \exp \left(\frac{C_{2}}{\lambda_{1}^{2}}|f|^{4}\right)|f|^{6}+2|f|^{2}\right) \\
\int_{-\infty}^{0}\left|\mathrm{~A}^{\frac{\alpha}{2}} f(r)\right|_{\mathrm{H}}^{2} \exp \left(C \frac{2 C_{2}}{\lambda_{1}^{2}} \exp \left(\frac{C_{2}}{\lambda_{1}^{2}}|f|^{4}\right)|f|^{6}+2|f|^{2}\right) .
\end{aligned}
$$

The proof is now complete.

Proposition A.3. Assume that $T \in \mathbb{R} \cup\{+\infty\}$. If $z$ belongs to $W^{1,2}(-\infty, T)$ then

$$
\lim _{t \rightarrow-\infty}|z(t)|_{\mathrm{V}}=0 \text {. }
$$


Proof. In view of [30, Theorem 2.2, p. 13] it is enough to consider the case $T=+\infty$. So let us take $z \in W^{1,2}(-\infty, \infty)$ Then, since $\mathrm{V}=[D(\mathrm{~A}), \mathrm{H}]_{1 / 2}$, according to [30, Theorem 3.1, p. $19], z: \mathbb{R} \rightarrow \mathrm{V}$ is a bounded and continuous function. Moreover, by [30, (2.27), p. 16]

$$
\lim _{t \rightarrow \pm \infty}|u(t)|_{\mid r H}=0 .
$$

Hence the result follows by applying (A.1).

\section{Appendix B. Proofs of Lemmas in Section 9}

Proof of Lemma 9.5. In view of Proposition [3.10, for any $\kappa>0$ there exists $T(\beta, \kappa, \mu)>0$ such that

$$
u^{\phi}(t ; 0) \in B_{\frac{\beta+1}{2}}(\kappa), \quad \phi \in B_{0}(\mu), \quad t>T(\beta, \kappa, \mu) .
$$

Thus, if we set $T_{1}=T(\beta, \kappa, \mu)+1$ and

$$
z_{1}(t)=u^{\phi}(t ; 0), \quad t \in\left[0, T_{1}\right]
$$

we have that $z_{1}(0)=\phi, z_{1}\left(T_{1}\right) \in D\left(\mathrm{~A}^{\frac{1+\beta}{2}}\right)$ and

$$
S_{T_{1}}^{\delta}\left(z_{1}\right)=0 .
$$

Now, we define

$$
z_{2}(t)=\left(T_{1}+1-t\right) e^{-\left(t-T_{1}\right) \mathrm{A}} z_{1}\left(T_{1}\right), \quad t \in\left[T_{1}, T_{1}+1\right] .
$$

We have $z_{2}\left(T_{1}\right)=z_{1}\left(T_{1}\right)$ and $z_{1}\left(T_{1}+1\right)=0$. Moreover,

$$
\mathcal{H}\left(z_{2}\right)(t)=-e^{-\left(t-T_{1}\right) \mathrm{A}} z_{1}\left(T_{1}\right)+B\left(z_{2}(t), z_{2}(t)\right),
$$

so that, according to Assumption 5.1, we have

$$
S_{T_{1}, T_{1}+1}^{\delta}\left(z_{2}\right) \leq c \int_{T_{1}}^{T_{1}+1}\left|e^{-\left(t-T_{1}\right) \mathrm{A}} z_{1}\left(T_{1}\right)\right|_{D\left(\mathrm{~A}^{\frac{\beta}{2}}\right)}^{2} d t+c \int_{T_{1}}^{T_{1}+1}\left|B\left(z_{2}(t), z_{2}(t)\right)\right|_{D\left(\mathrm{~A}^{\frac{\beta}{2}}\right)}^{2} d t .
$$

Now, thanks to (2.20), with $s=1+\beta$, we have

$$
\left|B\left(z_{2}(t), z_{2}(t)\right)\right|_{D\left(\mathrm{~A}^{\frac{\beta}{2}}\right)} \leq\left|e^{-\left(t-T_{1}\right) \mathrm{A}} z_{1}\left(T_{1}\right)\right|_{D\left(\mathrm{~A}^{\frac{1+\beta}{2}}\right)}^{2} \leq c \kappa^{2},
$$

so that

$$
S_{T_{1}, T_{1}+1}^{\delta}\left(z_{2}\right) \leq c \kappa^{2}+c \kappa^{4}
$$

Therefore, we fix $\bar{\kappa}>0$ small enough such that

$$
S_{T_{1}, T_{1}+1}^{\delta}\left(z_{2}\right) \leq c \bar{\kappa}^{2}+c \bar{\kappa}^{4}<\frac{\eta}{2},
$$

and in correspondence of $\bar{\kappa}$ we fix $T_{1}=T(\beta, \bar{\kappa}, \mu)$.

As we have seen in Lemma 9.3 , there exists $\phi_{\delta} \in \partial D \cap D\left(\mathrm{~A}^{\frac{1+\beta}{2}}\right)$ such that

$$
U_{\delta}\left(\phi_{\delta}\right)=\inf _{\phi \in \partial D} U_{\delta}(\phi) .
$$

According to Assumption 9.1, we can fix $\left\{\phi_{\delta, n}\right\}_{n \in \mathbb{N}} \subset \bar{D}^{c} \cap D\left(\mathrm{~A}^{\frac{1+\beta}{2}}\right)$, such that

$$
\lim _{n \rightarrow \infty}\left|\phi_{\delta, n}-\phi_{\delta}\right|_{D\left(\mathrm{~A}^{\frac{1+\beta}{2}}\right)}=0 \text {. }
$$


Thus, since the mapping $U_{\delta}: D\left(\mathrm{~A}^{\frac{1+\beta}{2}}\right) \rightarrow[0,+\infty)$ is continuous (see Proposition 6.6), we have

$$
\lim _{n \rightarrow \infty} U_{\delta}\left(\phi_{\delta, n}\right)=U_{\delta}\left(\phi_{\delta}\right)
$$

This means that we can fix $\bar{n} \in \mathbb{N}$ such that

$$
U_{\delta}\left(\phi_{\delta, \bar{n}}\right)<U_{\delta}\left(\phi_{\delta}\right)+\frac{\eta}{4},
$$

and $T_{2}=T_{2}(\eta)>0$ and $z_{3} \in C\left(\left[0, T_{2}\right] ; H\right)$ such that $z_{3}(0)=0, z_{3}\left(T_{2}\right)=\phi_{\delta, \bar{n}}$ and

$$
S_{T_{2}}^{\delta}\left(z_{3}\right)<U_{\delta}\left(\phi_{\delta, \bar{n}}\right)+\frac{\eta}{4}<U_{\delta}\left(\phi_{\delta}\right)+\frac{\eta}{2} .
$$

Therefore, if we define $T:=T_{1}+T_{2}+1$ and

$$
v^{\phi}(t)= \begin{cases}z_{1}(t), & t \in\left[0, T_{1}\right], \\ z_{2}(t), & t \in\left[T_{1}, T_{1}+1\right], \\ z_{3}\left(t-\left(T_{1}+1\right)\right), & t \in\left[T_{1}+1, T\right],\end{cases}
$$

we get $v^{\phi} \in C\left([0, T ; \mathrm{H})\right.$, with $v^{\phi}(0)=\phi$ and $v^{\phi}(T)=\phi_{\delta, \bar{n}}$ and

$$
S_{T}^{\delta}\left(v^{\phi}\right) \leq U_{\delta}\left(\phi_{\delta}\right)+\eta
$$

Moreover, $T$ only depends on $\mu$ and $\eta$.

Proof of Lemma 9.6 and lemma 9.7. The proofs of these two lemmas are analogous to the proofs of [11, Lemmas 7.3, 7.4 and 7.5] and is based on the validity of a large deviation principle for the 2-D Navier-Stokes equation perturbed by additive noise, as proved in Theorem 5.3, which is uniform with respect to the initial condition $\phi$ in a bounded set of $H$. The arguments used in [11 are an adaptation to an infinite dimensional setting of the methods used in [19, Chapter 5].

Proof of Lemma 9.8. Our proof follows a path analogous to the one followed in the proof of Lemmas 9.5, 9.6 and 9.9 in [12]. We proceed here in three steps.

Step 1. We will show that there exists a strictly increasing continuous function $\varphi:[0, \infty) \rightarrow$ $[0, \infty)$ such that for any $\phi \in \mathrm{H}$ and $f \in L^{2}(0, T ; H)$ and for any $T>0$,

$$
\left|u_{\phi}^{f}-u_{0}^{f}\right|_{C([0, T] ; H)} \leq \varphi\left(|f|_{L^{2}(0, T ; \mathrm{H})}\right)|\phi|_{\mathrm{H}},
$$

where $u_{\phi}^{f} \in L^{2}(0, T ; \mathrm{V})$, with $\left(u_{\phi}^{f}\right)^{\prime} \in L^{2}\left(0, T ; \mathrm{V}^{\prime}\right)$, is the solution of problem (3.1) , i.e.

$$
u^{\prime}(t)+\mathrm{A} u(t)+B(u(t), u(t))=f(t), \quad u(0)=\phi .
$$

Proof. Let us fix $T>0, \phi \in \mathrm{H}$ and $f \in L^{2}(0, T ; \mathrm{H})$, and denote $v:=u_{0}^{f}-u_{\phi}^{f}$. Then

$$
v^{\prime}(t)+\mathrm{A} v(t)+\mathrm{B}\left(v(t), u_{f}^{0}(t)\right)+\mathrm{B}\left(u_{f}^{0}(t), v(t)\right)=0, \quad v(0)=-\phi
$$


and hence, by [42, Lemmata III.2.1 and III.3.2],

$$
\begin{aligned}
\frac{1}{2} \frac{d}{d t}|v(t)|_{\mathrm{H}}^{2} & \left.+|v(t)|_{V}^{2}=-\left\langle\mathrm{B}\left(v(t), u_{f}^{0}(t)\right), v(t)\right\rangle_{\mathrm{H}} \leq \sqrt{2} \mid u_{f}^{0}(t)\right)\left.\right|_{V}|v(t)|_{\mathrm{H}}|v(t)|_{V} \\
& \left.\leq \frac{1}{2}|v(t)|_{V}^{2}+\mid u_{f}^{0}(t)\right)\left.\right|_{V} ^{2}|v(t)|_{\mathrm{H}}^{2} .
\end{aligned}
$$

By the Gronwall Lemma, this implies

$$
\left|u_{\phi}^{f}(t)-u_{0}^{f}(t)\right|_{\mathrm{H}}^{2} \leq|\phi|_{\mathrm{H}}^{2} \exp \left(2 \int_{0}^{t}\left|u_{f}^{0}(s)\right|_{V}^{2} d s\right), \quad t \in[0, T] .
$$

This together with inequality (3.2) implies that

$$
\left|u_{\phi}^{f}-u_{0}^{f}\right|_{C([0, T] ; \mathrm{H})} \leq|\phi|_{\mathrm{H}} \varphi\left(|f|_{L^{2}\left(0, T ; \mathrm{V}^{\prime}\right)}^{2}\right) .
$$

Step 2. Assume that $C \subset \mathrm{H}$ is a closed set and a real number $\beta$ satisfies

$$
\beta<\inf _{\phi \in C} U_{\delta}(\phi) .
$$

Then there exists a positive number $\rho_{0}>0$ such that for every $T>0$ and every $u \in$ $C([0, T] ; \mathrm{H})$, with $|u(0)|_{\mathrm{H}}<\rho_{0}$ and $S_{0, T}^{\delta}(u) \leq \beta$, it holds

$$
\operatorname{dist}_{\mathrm{H}}(u(t), C)>\rho_{0}, \text { for every } t \in[0, T] \text {. }
$$

Proof. Suppose our claim is not true. Then for every $n \in \mathbb{N}$ we can find $\phi_{n} \in \mathrm{H}, T_{n}>0$, $\hat{T}_{n} \in\left[0, T_{n}\right]$ and $f_{n} \in L^{2}\left(0, T_{n} ; H\right)$ such that for every $n \in \mathbb{N}$

$$
\left|\phi_{n}\right|_{\mathrm{H}}<\frac{1}{n}, \quad\left|f_{n}\right|_{L^{2}\left(0, T_{n} ; \mathrm{H}\right)}^{2}<2 \beta
$$

and

$$
\operatorname{dist}\left(u_{\phi_{n}}^{f_{n}}\left(\hat{T}_{n}\right), C\right) \leq \frac{1}{n} .
$$

Now, if we set $\hat{\phi}_{n}:=u_{0}^{f_{n}}\left(\hat{T}_{n}\right)$, then by $(\underline{\mathrm{B} .4})$ we have

$$
\left|\hat{\phi}_{n}-u_{\phi_{n}}^{f_{n}}\left(\hat{T}_{n}\right)\right|_{\mathrm{H}} \leq \varphi\left(\left|f_{n}\right|_{L^{2}\left(0, T_{n} ; \mathrm{H}\right)}\right)\left|\phi_{n}\right|_{\mathrm{H}},
$$

so that, thanks to (B.9) and (B.10), we get

$$
\lim _{n \rightarrow \infty} \operatorname{dist}\left(\hat{\phi}_{n}, C\right) \leq \lim _{n \rightarrow \infty}\left[\varphi(\sqrt{2 \beta})\left|\phi_{n}\right|_{\mathrm{H}}+\frac{1}{n}\right]=0 \text {. }
$$

Moreover,

$$
U_{\delta}\left(\hat{\phi}_{n}\right) \leq \frac{1}{2}\left|f_{n}\right|_{L^{2}\left(0, T_{n} ; \mathrm{H}\right)}^{2} \leq \beta,
$$

and then, by the compactness in $\mathrm{H}$ of the level sets of the functional $U_{\delta}$, we infer that there is a subsequence $\left\{\hat{\phi}_{n_{k}}\right\}$ and an element $\hat{\phi} \in \mathrm{H}$ such that $\hat{\phi}_{n_{k}} \rightarrow \hat{\phi}$ in $\mathrm{H}$ and $U_{\delta}(\hat{\phi}) \leq \beta$. On the other hand, $C$ is a closed subset of $\mathrm{H}$ so by (B.11) we infer that $\hat{\phi} \in C$. This contradicts our assumption (B.8).

Step 3. Assume that $N \subset \partial D$ is a closed set. Then (9.6) holds. 
Proof. Let us choose a real number $\beta$ such that condition (B.8) holds. Let us also choose a positive number $\mu>0$ such that $B_{0}(3 \mu) \subset D$. For any $T>0$, we have

$$
\mathbb{P}\left(u_{\phi}^{\varepsilon, \delta}\left(\sigma_{\phi}^{\varepsilon, \delta, \mu}\right) \in N\right) \leq \mathbb{P}\left(\sigma_{\phi}^{\varepsilon, \delta, \mu}>T\right)+\mathbb{P}\left(u_{\phi}^{\varepsilon, \delta}(t) \in N \text {, for some } t \in[0, T]\right) .
$$

According to Step 2 and to the fact that the large deviation principle proved in Theorem 5.3 is uniform with respect to initial conditions $\phi$ in bounded sets of $\mathrm{H}$, for any $\mu>0$ such that $B_{0}(3 \mu) \subset D$ and any $\beta<\inf _{x \in C} U_{\delta}(x)$, we can find $\varepsilon_{1}>0$ such that for every $\varepsilon \in\left(0, \varepsilon_{1}\right]$

$$
\begin{aligned}
\sup _{\phi \in B_{0}(3 \mu)} & \mathbb{P}\left(u_{\phi}^{\varepsilon, \delta}(t) \in N, \text { for some } t \in[0, T]\right) \\
\leq & \sup _{\phi \in B_{0}(3 \mu)} \mathbb{P}\left(\operatorname{dist}_{C([0, T] ; \mathrm{H})}\left(u_{\phi}^{\varepsilon, \delta}, K_{T}^{\delta}(\beta)\right)>3 \mu\right) \leq e^{-\frac{\beta-\gamma}{\varepsilon}},
\end{aligned}
$$

where $K_{T}^{\delta}(\beta)=\left\{u \in C([0, T] ; \mathrm{H}): S_{T}^{\delta}(u) \leq \beta\right\}$.

Moreover, in view of Lemma 9.7, there exist $T>0$ and $\varepsilon_{2} \leq \varepsilon_{1}$ such that

$$
\sup _{x \in D} \mathbb{P}\left(\sigma_{\phi}^{\varepsilon, \delta, \mu}>T\right) \leq e^{-\frac{\beta}{\varepsilon}}, \quad \varepsilon \leq \varepsilon_{2} .
$$

Then, thanks to $(\underline{\mathrm{B} .12})$, we can conclude the proof of Step 3, due to the arbitrariness of $\gamma>0$ and condition (B.8).

Proof of Lemma 9.9. Let us fix $\phi \in \mathrm{H}$ and $\delta \in(0,1]$. For any $\varepsilon>0$, let us now denote by $z_{\varepsilon, \delta}$ the Ornstein-Uhlenbeck process defined by equation (5.4) and by $u_{\phi}^{\varepsilon, \delta}$ the solution to the stochastic Navier-Stokes equation (5.1). Thanks to [10, Theorem 1.2 ] (with $\xi(t)$ being the $\gamma$-radonifying natural embedding operator from $Q_{\delta}(\mathrm{H})$ to $\mathrm{H} \cap L^{4}(\mathcal{O})$ ) we infer that there exists a constant $C>0$ such that for any $R>0$ and $\varepsilon>0$

$$
\varepsilon \log \mathbb{P}\left(\left|z^{\varepsilon, \delta}\right|_{C\left([0, T] ; L^{4}(\mathcal{O})\right)} \geq R\right) \leq-\frac{R^{2}}{C T} .
$$

Let us now fix $\mu>0$ and $\lambda>0$. By the above inequality there exists $T_{0}>0$ such that

$$
\varepsilon \log \mathbb{P}\left(\left|z^{\varepsilon, \delta}\right|_{C\left(\left[0, T_{0}\right] ; L^{4}(\mathcal{O})\right)} \geq \frac{\mu}{3}\right) \leq-\frac{\lambda}{2}, \quad \varepsilon>0 .
$$

For a given $z \in C\left(\left[0, T_{0}\right] ; L^{4}(\mathcal{O})\right.$ and $\phi \in \mathrm{H}$ let us denote by $v_{\phi}^{z}$ the unique solution to the problem

$$
\left(v_{\phi}^{z}\right)^{\prime}(t)+\mathrm{A} v_{\phi}^{z}(t)+B\left(v_{\phi}^{z}(t)+z(t), v_{\phi}^{z}(t)+z(t)\right)=0, \quad t \in\left[0, T_{0}\right], \quad v_{\phi}^{z}(0)=\phi .
$$

Note that $v_{\phi}^{0}$ is the unique solution to the deterministic NSE satisfying the initial condition $v_{\phi}^{0}(0)=\phi$. Hence

$$
\sup _{\phi \in B_{0}(\mu)}\left|v_{\phi}^{0}-\phi\right|_{C([0, T] ; \mathrm{H})} \leq 2 \mu .
$$

By [9, Theorem 4.6] we infer that there exists $\beta>0$ such that

$$
|z|_{C\left(\left[0, T_{0}\right] ; L^{4}(\mathcal{O})\right)}<\beta \Longrightarrow \sup _{\phi \in B_{0}(\mu)}\left|v_{\phi}^{z}-v_{\phi}^{0}\right|_{C\left(\left[0, T_{0}\right] ; \mathrm{H}\right)}<\frac{\mu}{3} .
$$


By a simple uniqueness argument, the above holds with the same constant for all $T \in\left(0, T_{0}\right]$, i.e. there exists $\beta>0$ such that for every $T \in\left(0, T_{0}\right]$,

$$
\begin{aligned}
& |z|_{C\left([0, T] ; L^{4}(\mathcal{O})\right)}<\beta \Longrightarrow \sup _{\phi \in B_{0}(\mu)}\left|v_{\phi}^{z}-v_{\phi}^{0}\right|_{C([0, T] ; \mathrm{H})}<\frac{\mu}{3}, \\
& \sup _{\phi \in B_{0}(\mu)}\left|v_{\phi}^{0}-\phi\right|_{C([0, T] ; \mathrm{H})}<2 \mu .
\end{aligned}
$$

Since, see [9], $u_{\phi}^{\varepsilon, \delta}-\phi=z^{\varepsilon, \delta}+v_{\phi}^{z^{\varepsilon, \delta}}-v_{\phi}^{0}+v_{\phi}^{0}-\phi$, we infer that for every $\varepsilon>0$,

$$
\begin{aligned}
& \varepsilon \log \sup _{\phi \in B_{0}(\mu)} \mathbb{P}\left(\left|u_{\phi}^{\varepsilon, \delta}-\phi\right|_{C([0, T] ; \mathrm{H})} \geq 3 \mu\right) \leq \varepsilon \log \mathbb{P}\left(\left|z^{\varepsilon, \delta}\right|_{C([0, T] ; \mathrm{H})} \geq \frac{\mu}{3}\right) \\
& +\varepsilon \log \sup _{\phi \in B_{0}(\mu)} \mathbb{P}\left(\left|v_{\phi}^{z^{\varepsilon, \delta}}-v_{\phi}^{0}\right|_{C([0, T] ; \mathrm{H})} \geq \frac{\mu}{3}\right)+\varepsilon \log \sup _{\phi \in B_{0}(\mu)} \mathbb{P}\left(\left|v_{\phi}^{0}-\phi\right|_{C([0, T] ; \mathrm{H})} \geq \frac{7 \mu}{3}\right) .
\end{aligned}
$$

Let us note that by the second part of (B.20), the last term on the RHS of inequality (B.21) is equal to 0 .

In order to estimate the first term on the RHS of inequality (B.21) let us choose $T \leq T_{0}$ such that

$$
\frac{\beta^{2}}{C T} \geq \frac{\lambda}{2}
$$

and then apply inequality (B.15) with $R=\beta$. We get that $\varepsilon \log \mathbb{P}\left(\left|z^{\varepsilon, \delta}\right|_{C([0, T] ; \mathrm{H})} \geq \frac{\mu}{3}\right) \leq-\frac{\lambda}{2}$. In order to estimate the second term on the RHS of inequality (B.21) we use inequalities (B.20) and (B.16). Thus we deduce that

$$
\begin{aligned}
& \varepsilon \log \sup _{\phi \in B_{0}(\mu)} \mathbb{P}\left(\left|u_{\phi}^{\varepsilon, \delta}-\phi\right|_{C([0, T] ; \mathrm{H})} \geq \mu\right) \leq \varepsilon \log \mathbb{P}\left(\left|z^{\varepsilon, \delta}\right|_{C([0, T] ; \mathrm{H})} \geq \frac{\mu}{3}\right) \\
& +\varepsilon \log \mathbb{P}\left(\left|z^{\varepsilon, \delta}\right|_{C\left(\left[0, T_{0}\right] ; L^{4}(\mathcal{O})\right)} \geq \beta\right) \leq-\frac{\lambda}{2}-\frac{\lambda}{2}=-\lambda .
\end{aligned}
$$

This completes the proof.

\section{REFERENCES}

[1] H. Amann, Multiplication in Sobolev and Besov Spaces, pp. 27-50 in Quaderni "Nonlinear Analysis", A tribute in Honour of Giovanni Prodi, SNS Pisa, Pisa 1991

[2] J. Bergh, L. Löfström, Interpolation SPACES. An introduction. Grundlehren der Mathematischen Wissenschaften, No. 223, Springer-Verlag, Berlin-New York, 1976.

[3] F. Bouchet, J. Laurie, O. Zaboronski, Langevin dynamics, large deviations and instantons for the quasigeostrophic model and two-dimensional Euler equations, arXiv:1403.0216

[4] Z. Brzeźniak, Stochastic Partial Differential Equations in M-type 2 Banach spaces, Potential Analysis 4, no. 1, 1-47 (1995)

[5] Z. Brzeźniak, On analytic dependence of solutions of Navier-Stokes equations with respect to exterior force and initial velocity, Univ. Iagel. Acta Math. No. 28 (1991), 111-124.

[6] Z. Brzeźniak, On Sobolev and Besov spaces regularity of Brownian paths, Stochastics and Stochastics Reports 56, 1-15 (1996)

[7] Z. Brzeźniak, On stochastic convolution in Banach spaces and applications, Stochastics and Stochastics Reports 61, pp. 245-295 (1997) 
[8] Z. Brzeźniak and D. Gątarek, Martingale solutions and invariant measures for stochastic evolution equations in Banach spaces, Stochastic Process. Appl. 84, no. 2, 187âĂŞ225 (1999)

[9] Z. Brzeźniak and Y. Li, Asymptotic compactness and absorbing sets for 2D stochastic Navier-Stokes equations on some unbounded domains, Trans. Amer. Math. Soc. 358, no. 12, 5587-5629 (2006).

[10] Z. Brzeńiak and S. Peszat, Maximal inequalities and exponential estimates for stochastic convolutions in Banach spaces, in Stochastic Processes, Physics AND GeOMETRY: NEW InTERPlays, I (Leipzig, 1999), CMS Conf. Proc., 28, Amer. Math. Soc., Providence, RI, 2000.

[11] S. Cerrai and M. Freidlin, Approximation of quasi-potentials and exit problems for multidimensional RDE's with noise, Transactions of the AMS 363 (2011), pp. 3853-3892.

[12] S. Cerrai and M. Salins, Smoluchowski-Kramers approximation and large deviations for non-gradient systems with an infinite number of degrees of freedom and applications to the exit problem, arXiv:1403.5743,

[13] L. Cattabriga, Su un problema al contorno relativo al sistema di equazioni di Stokes, Rend. Sem. Mat. Univ. Padova 31, 308-340 (1961).

[14] F. Chenal, A. Millet, Uniform large deviations for parabolic SPDE's and applications, Stochastic Processes and their Applications 72 (1997), pp. 161-186.

[15] G. Dal Maso, An introduction to Gamma convergence, Progress in Nonlinear Differential Equations and their Applications, Birkhauser Boston, Inc., Boston, MA, 1993.

[16] G. Da Prato and A. Debussche, Two-dimensional Navier-Stokes equations driven by a space-time white noise, J. Funct. Anal. 196, no. 1, 180-210 (2002)

[17] G. Da Prato, A.J. Pritchard and J. Zabczyk, On minimum energy problems. SIAM J. Control Optim. 29, no. 1, 209-221 (1991)

[18] G. Da Prato and J. Zabczyk, Stochastic equations in infinite dimensions, Cambridge Univ. Press, Cambridge, 1992.

[19] A. Dembo, O. Zeitouni, Large Deviations Techniques and Applications, Second Edition, Springer Verlag (1998).

[20] M.I. Freidlin, A.D. Wentzell, Random perturbations of dynamical systems, Second edition, Springer-Verlag, New York (1998).

[21] F. Flandoli, Dissipativity and invariant measures for stochastic Navier-Stokes equations, NoDEA 1, 403423 (1994).

[22] F. Flandoli, D. Gątarek, Martingale and stationary solutions for stochastic Navier-Stokes equations, Probab. Theory Related Fields 102, no. 3, 367-391 (1995)

[23] F. Flandoli and F. Gozzi, Kolmogorov equation associated to a stochastic Navier-Stokes equation, J. Funct. Anal. 160, no. 1, 312-336 (1998)

[24] D. Fujiwara, and H. Morimoto, An $L_{r}$ theorem of the Helmhotz decomposition of vector fields, J. Fac. Sci. Univ. Tokyo Sect. IA Math. 24, 685-700 (1977)

[25] G. Furioli, P.G. Lemarié-Rieusset, E. Terraneo, Unicité dans $L^{3}\left(\mathbb{R}^{3}\right) L$ et d'autres espaces fonctionnels limites pour Navier-Stokes, Rev. Mat. Iberoamericana 16, no. 3, 605-667 (2000)

[26] D. Gątarek, B. Gołdys, On weak solutions of stochastic equations in Hilbert spaces, Stochastics Stochastics Rep. 46, no. 1-2, 41-51 (1994)

[27] J. G. Heywood, The Navier-Stokes equations: on the existence, regularity and decay of solutions, Indiana Univ. Math. J. 29, no. 5, 639-681 (1980)

[28] R.V. Kohn, M.G. Reznikoff and E. Vanden-Eijnden, Magnetic elements at finite temperature and large deviation theory. J. Nonlinear Sci. 15, 223-253 (2005)

[29] J.L. Lions, Quelques mèthodes de RÈSOlution Des Problèmes auX limites non linèaires, Dunod; Gauthier-Villars, Paris 1969

[30] J.L. Lions and E. Magenes, Non-homogeneous boundary value problems and applications. Vol. I, Grundlehren der Mathematischen Wissenschaften [Fundamental Principles of Mathematical Sciences], 181. Springer-Verlag, New York-Heidelberg, 1972. 
[31] P.-L. Lions, N. Masmoudi, Uniqueness of mild solutions of the Navier-Stokes system in $L^{N}$, Comm. Partial Differential Equations 26, no. 11-12, 2211-2226 (2001)

[32] M. Metivier, Stochastic Partial Differential EQuations in infinite-Dimensional spaces, with a preface by G. Da Prato. Scuola Normale Superiore di Pisa. Quaderni. Pisa, 1988.

[33] W. Rudin, Functional analysis. Second edition. International Series in Pure and Applied Mathematics. McGraw-Hill, Inc., New York, 1991.

[34] R. Seeley, Interpolation in $L^{p}$ with boundary conditions, in COLLECTION of ARTICLES HONORING THE COMPletion by Antoni Zygmund of 50 years of SCiEntific aCtivity, I, Studia Math., 44, 47-60 (1972)

[35] J. Simon, Sobolev, Besov and Nikolskii fractional spaces: imbeddings and comparisons for vector valued spaces on an interval, Ann. Mat. Pura Appl. 157, 117-148 (1990).

[36] S.S. Sritharan, P.Sundar, Large deviations for the two-dimensional Navier-Stokes equations with multiplicative noise, Stochastic Processes and their Applications 116, 1636-1659 (2006).

[37] M. Struwe, Variational methods. Applications to nonlinear partial differential equations and Hamiltonian systems. Fourth edition. A Series of Modern Surveys in Mathematics, 34. Springer-Verlag, Berlin, 2008

[38] M.E. Taylor, Pseudodifferential Operators, Princeton University Press, Princeton, New Jersey, 1981.

[39] R. Temam, Behaviour at time $t=0$ of the solutions of semilinear evolution equations. J. Differential Equations 43, no. 1, 73-92 (1982).

[40] R. Temam, Navier-Stokes equations and nonlinear Functional analysis, CBMS-NSF Regional Conference Series in Applied Mathematics, 41, Society for Industrial and Applied Mathematics (SIAM), Philadelphia, PA, 1983.

[41] R. Temam, Infinite Dimensioanal Dynamical Systems in Mechanics and Physics, second edition, Springer, New York. R. (1997).

[42] R. Temam, Navier-Stokes equations. Theory and numerical analysis, Reprint of the 1984 edition. AMS Chelsea Publishing, Providence, RI, 2001. xiv+408 pp.

[43] H. Triebel, Interpolation Theory, Function Spaces, Differential Operators, North-Holland, Amsterdam New York Oxford, 1978.

[44] H. Triebel, Interpolation theory, FUnCtion SPACES, Differential operators, 2nd ed., Johann Ambrosius Barth, Heidelberg, 1995.

[45] M. Viot, Solutions faibles aux équations aux dérivées partielles stochastiques non linéaires, Thése, Université Pierre et Marie Curie, Paris (1976).

[46] M.J. Vishik, A.V. Fursikov, Mathematical Problems of Statistical Hydromechanics, Kluwer Academic Publishers, Dordrecht, 1988.

[47] J. Zabczyk, On large deviations for stochastic evolution equations, Stochastic systems and optimization (Warsaw, 1988), Lecture Notes in Control and Inform. Sci., 136, Springer, Berlin, 240-253, (1989).

Department of Mathematics, The University of York, Heslington, York YO10 5DD, UK

E-mail address: zdzislaw.brzezniak@york.ac.uk

Department of Mathematics, University of Maryland, College Park, MD, 20742, USA

E-mail address: cerrai@math.umd.edu, mif@math.umd.edu 